\title{
Optical System Case Studies for Speckle Imaging
}

C. J. Carrano

Written Dec 2007

Released Oct 2013 


\section{Disclaimer}

This document was prepared as an account of work sponsored by an agency of the United States government. Neither the United States government nor Lawrence Livermore National Security, LLC, nor any of their employees makes any warranty, expressed or implied, or assumes any legal liability or responsibility for the accuracy, completeness, or usefulness of any information, apparatus, product, or process disclosed, or represents that its use would not infringe privately owned rights. Reference herein to any specific commercial product, process, or service by trade name, trademark, manufacturer, or otherwise does not necessarily constitute or imply its endorsement, recommendation, or favoring by the United States government or Lawrence Livermore National Security, LLC. The views and opinions of authors expressed herein do not necessarily state or reflect those of the United States government or Lawrence Livermore National Security, LLC, and shall not be used for advertising or product endorsement purposes.

\section{Auspices Statement}

This work performed under the auspices of the U.S. Department of Energy by Lawrence Livermore National Laboratory under Contract DE-AC52-07NA27344. 


\section{Optical system case studies \\ C. J. Carrano}

\section{Introduction}

The purpose of this document is to detail the effect of various optical and camera system parameters for two different classes of surveillance imaging scenarios. We will take into account the effects of expected atmospheric turbulence and demonstrate where image enhancement techniques such as speckle imaging[1] can help.

In the scenarios considered here, primary factors that determine the limiting resolutions are the range to target, wavelength, focal length, camera pixel size, and aperture size. Depending on the weather, imaging geometry, and the nature of the terrain below the imaging path, the atmospheric effects will vary. For the first scenario, we are interested in studying system parameters for imaging over shorter ranges from 2 to $8 \mathrm{~km}$ from very low slant angles over land or water. For the second scenario, we are interested in studying system parameters for imaging from $10 \mathrm{~km}$ to $60 \mathrm{~km}$ from low slant angles over land.

\subsection{Scenario 1}

For the first scenario, we will examine very low slant angles less than one degree, but not purely horizontal. Figure 1 depicts the geometry. Targets of interest at this range include people, signs, and identifying features on vehicles (e.g. license plates, other numbers, letters, or symbols.)

Camera

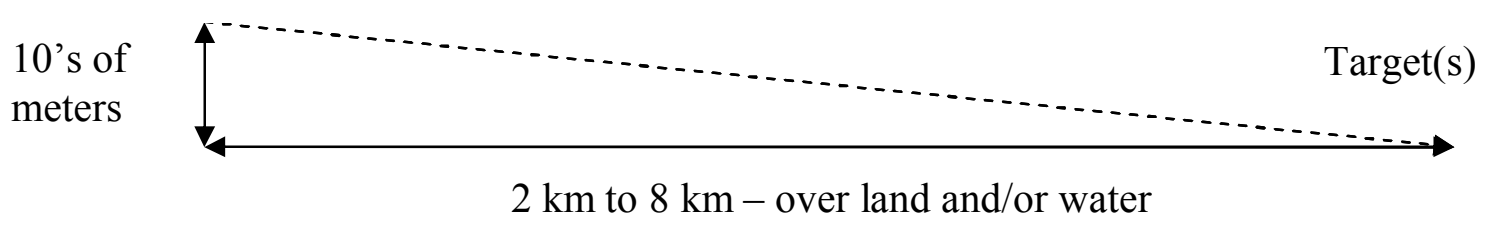

Figure 1: General imaging geometry of scenario 1. (Not to scale.)

Cameras and optics are most readily available and inexpensive in the visible wavelengths. In this section we will discuss the best resolution and or sampling that an optical system can obtain without atmosphere and then predict how much resolution degradation the atmosphere will produce. We will then show simulations of imagery that have been degraded to the predicted levels as well as results which have been processed and improved with our speckle imaging software. 


\subsection{Aperture alone - the diffraction limit}

Considering the diffraction limit of various sized apertures for the ranges of interest, the plot in Figure 2 represents the best that the optical system could ever do, neglecting camera sampling and target contrast. If the requirement was to resolve a $2 \mathrm{~cm}$ sized feature at a distance of $4 \mathrm{~km}$, an optic with a $10 \mathrm{~cm}$ diameter would be needed.

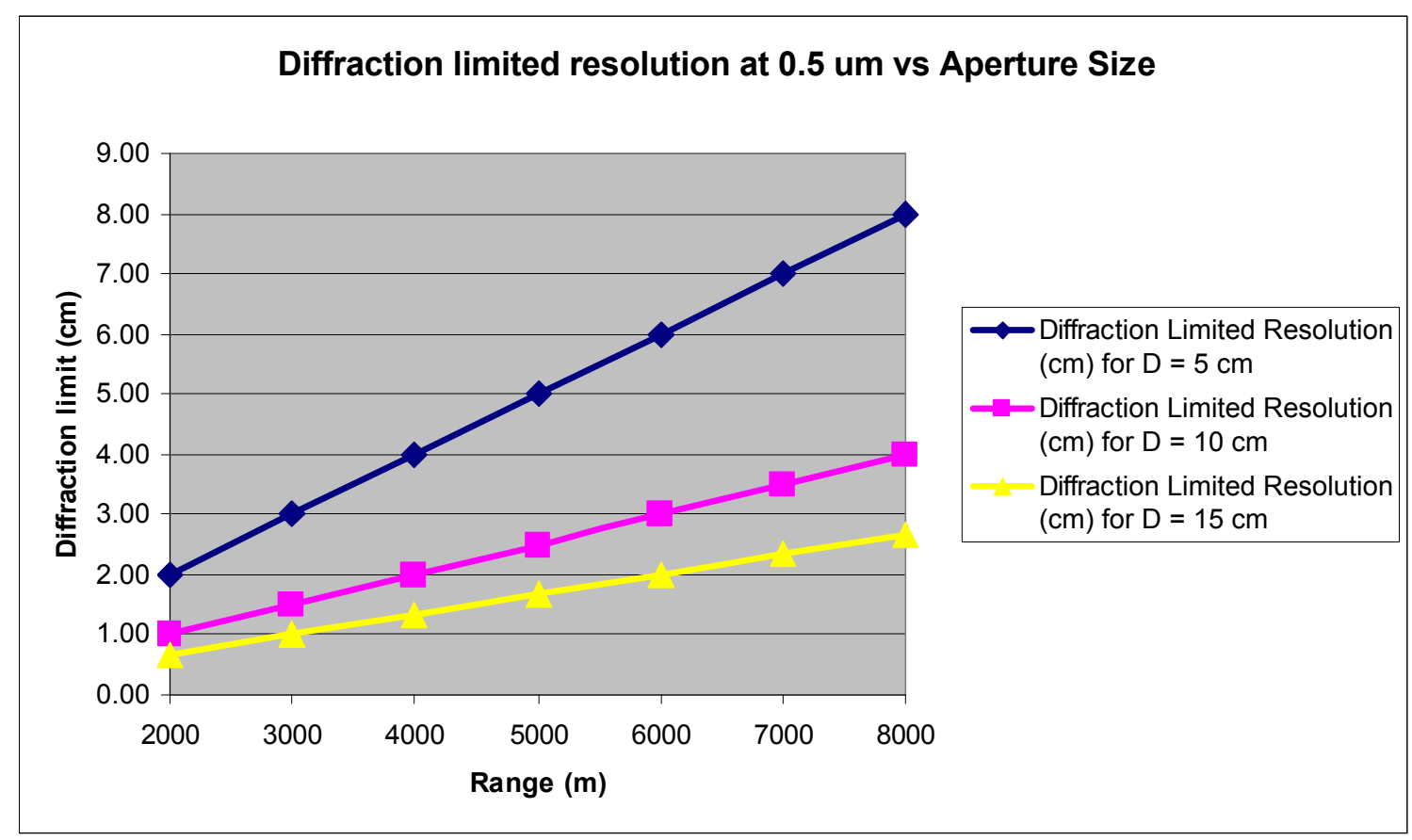

Figure 2: Plot of diffraction limited resolution of three aperture sizes vs range in the visible $(\lambda=0.5 \mu \mathrm{m})$. We calculate this using the formula ( $\lambda *$ Range)/D

\subsection{Focal length and camera pixel size}

It is only possible sample the diffraction limit of the optic if the focal length and camera pixel sizes are chosen appropriately. Depending on the minimum feature size of the targets we need to be able to see, the effective focal length of the optical system together with the camera pixel size determines the pixel sampling interval at range. To achieve the best resolution possible, it is best to sample at the Nyquist sampling interval for the aperture, which is one half the diffraction limit. Sampling any finer than that will not give additional information. See Figure 2 for the diffraction limited values for three aperture sizes versus range. Figure 3 shows the dependence of the effective focal length choice on the pixel size at range for various ranges. The calculations assume a camera CCD pixel size of 7.5 microns. We calculate the pixel-size-at-range using the formula (CCD pixel size * range / focal length), which means that the pixel-sizeat-range directly scales with CCD pixel size. If your camera has a pixel size of $15 \mu \mathrm{m}$, then these numbers double. There may be other reasons not to sample too finely including field of view, light levels, and target contrast considerations. 
For example, as the pixel-size-at-range decreases, the field-of-view of the resulting imagery is decreased as well as the amount of light on a pixel which increases noise. With increased noise the ability to see low contrast targets becomes worse.

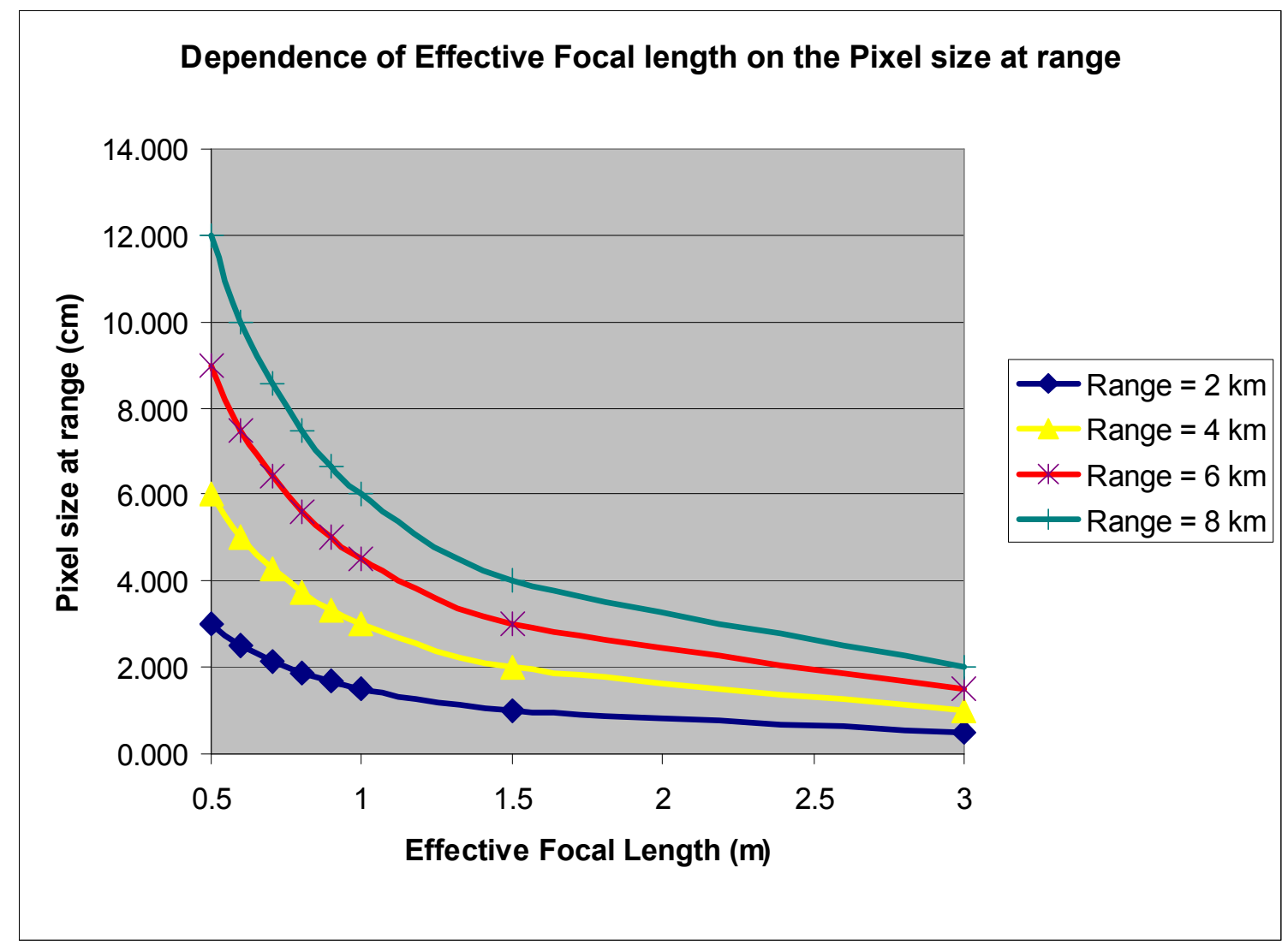

Figure 3: Plot of the pixel size in $\mathrm{cm}$ at range versus focal length and range for a camera with CCD pixel size of $7.5 \mu \mathrm{m}$ per pixel.

\subsection{Atmospheric effects}

Even with the best sampling and largest apertures, the quality of the imagery will be limited by atmospheric effects in the regime of this low slant path imaging scenario. Depending on the path length and the details of topology below the imaging path, the characteristic resolution will vary. The number often used to characterize the amount of blurring we see is atmospheric coherence length $\left(r_{0}\right)$. $R_{0}$ determines the effective resolution that the optic can see. Instead of $\lambda / D$, the effective resolution becomes $\lambda / r_{0}$. The isoplanatic angle $\left(\theta_{0}\right)$ is also another factor that affects the image quality, mostly in terms of warping and distortion. The isoplanatic angle is a measure of the angle over which the mean squared phase error seen by two points over their optical path to the telescope is 1 radian squared. If $\theta_{0}$ is very small compared to the field-of-view, then we are operating in an anisoplanatic regime and all the methods and algorithms that require isoplanicity will fail or work poorly (e.g. single-conjugate adaptive-optics). 
While it is nearly impossible to predict $r_{0}$ accurately without complete knowledge of the refractive index structure parameter, $\mathrm{C}_{\mathrm{n}}{ }^{2}$, over the entire imaging path, we can estimate it based on past measurements in similar conditions and employ the standard $\mathrm{h}^{-4 / 3}$ model for how $\mathrm{C}_{\mathrm{n}}{ }^{2}$ varies with altitude. The integral in Equation 1 describes the relationship between $\mathrm{C}_{\mathrm{n}}{ }^{2}$ and $\mathrm{r} 0$.

$$
r_{0}=2.1\left[1.46 k^{2} \int_{0}^{L} C n^{2}(h)\left(\frac{L-h}{L}\right)^{5 / 3} d h\right]^{-3 / 5}
$$

Where $h$ is the turbulent layer distance, dh is thickness element, $L$ is total distance from camera to target, and $\mathrm{k}$ is the optical wavenumber.

If there is a choice for what time of day to do the imaging, choosing times around sunset and sunrise are the best because $\mathrm{C}_{n}{ }^{2}$ is lowest during those times. Over land, $\mathrm{C}_{\mathrm{n}}{ }^{2}$ tends to be higher than over water, but both cases are dependent on altitude, temperature, wind speed and relative humidity. Near the surface $\mathrm{C}_{n}{ }^{2}$ can be as low as a few times $10^{-15} \mathrm{~m}^{-2 / 3}$ at sunset/sunrise, but can rise to several times $10^{-11} \mathrm{~m}^{-2 / 3}$ during the day in the worst of conditions.

The plot in Figure $4 a$ illustrates a realistic prediction of $r_{0}$ versus range for three different conditions. The geometry is for a simple slant path looking down from $15 \mathrm{~m}$ altitude. For the weaker turbulence case $\mathrm{C}_{\mathrm{n}}{ }^{2}$ at ground level is taken to be $5 \times 10^{-14} \mathrm{~m}^{-2 / 3}$, while for the medium turbulence case it is $1 \times 10^{-13} \mathrm{~m}^{-2 / 3}$ and for the stronger turbulence case it is set to $1 \times 10^{-12} \mathrm{~m}^{-2 / 3}$.

Using the $r_{0}$ estimates together with the aperture sizes, we can estimate the image resolution degradation from the diffraction limit expected versus range. This is shown in Figure $4 \mathrm{~b}$. Alternatively, we can think of this plot as the maximum amount of image resolution improvement to be gained using image resolution enhancement techniques.

As with any image enhancement technique, it may or may not obtain the full improvement back to the diffraction limited resolution. The performance of the algorithm will depend on several factors, including anisoplanatism (nonuniformity of the turbulence) and scintillation which gets worse as the turbulence get stronger, contrast of the target and noise factors within the camera. For a treatment of how speckle image processing quality degrades with isoplanatic angle for Nyquist sampled imagery see Ref [2]. The effect of anisoplanatism on the raw data frames is to distort and warp the data because different regions of the scene are passing through different phase distortions. Because the speckle algorithm was formulated with the assumption of a uniform phase distortion, there is some price to pay for non-uniform phase distortion, but the algorithm appears to be able to handle quite a bit of it. Much of the phase non-uniformity is mitigated by independently speckle processing small regions of the data and merging them together at the end, but in medium to strong turbulence $\left(D / r_{0}>7\right)$ it starts to have a noticeable effect, because you can't pick a region size small enough to both accommodate small isoplanatic angles and wide point spread functions $\left(D / r_{0}\right)$. Based on simulations we have done as well as experimental observation, a first order approximation to the expected image quality versus the ratio of the diffraction limit to the isoplanatic angle is given Figure 5. Depending on $\mathrm{D} / \mathrm{r} 0$, the image quality may only degrade a small amount or a larger amount, 
but it never gets worse than the shift-and-add image in terms of resolution. Although ignored here for simplicity, as the isoplanatic angle approaches the diffraction limit, other effects not accounted for here start to surface such as wispy artifacts in the processed image.

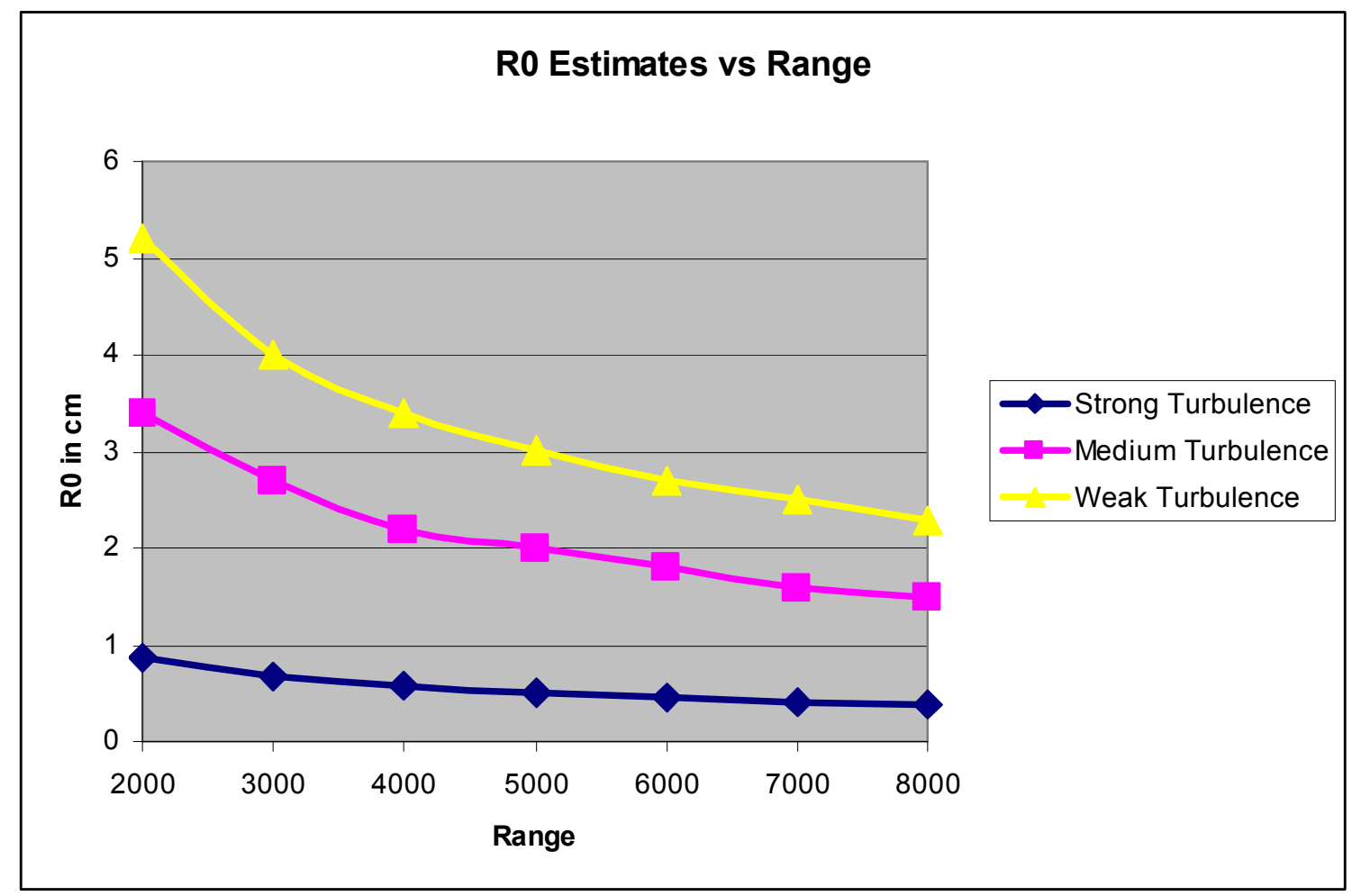

Figure 4a: $R_{0}$ estimates for typical values of $C_{n}{ }^{2}$ for a slant path from 15 meters altitude looking down. 


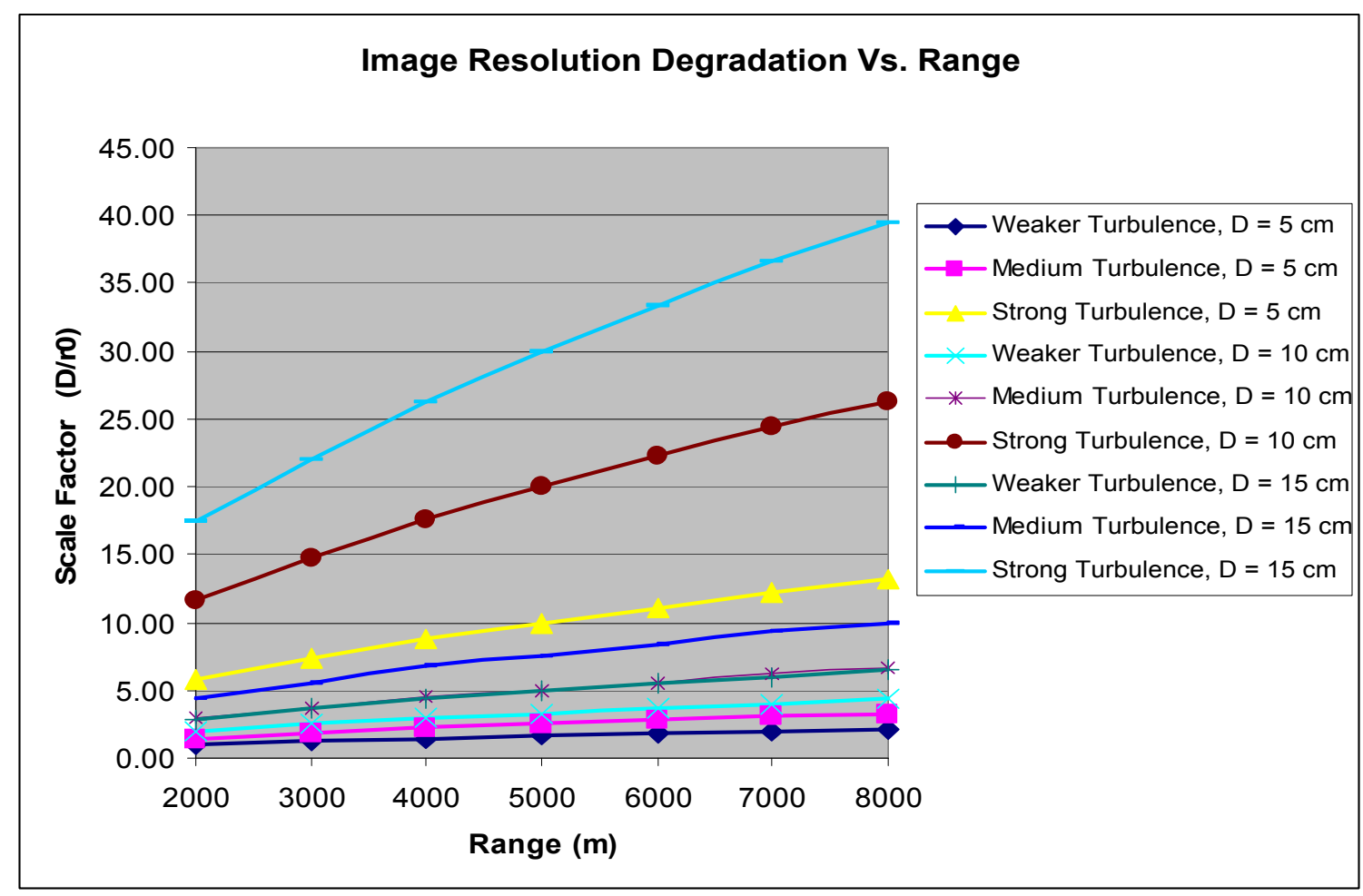

Figure 4b: Image resolution degradation (or needed improvement) scale factor vs range for three aperture sizes over using the $\mathrm{r} 0$ values from Figure $4 \mathrm{a}$.

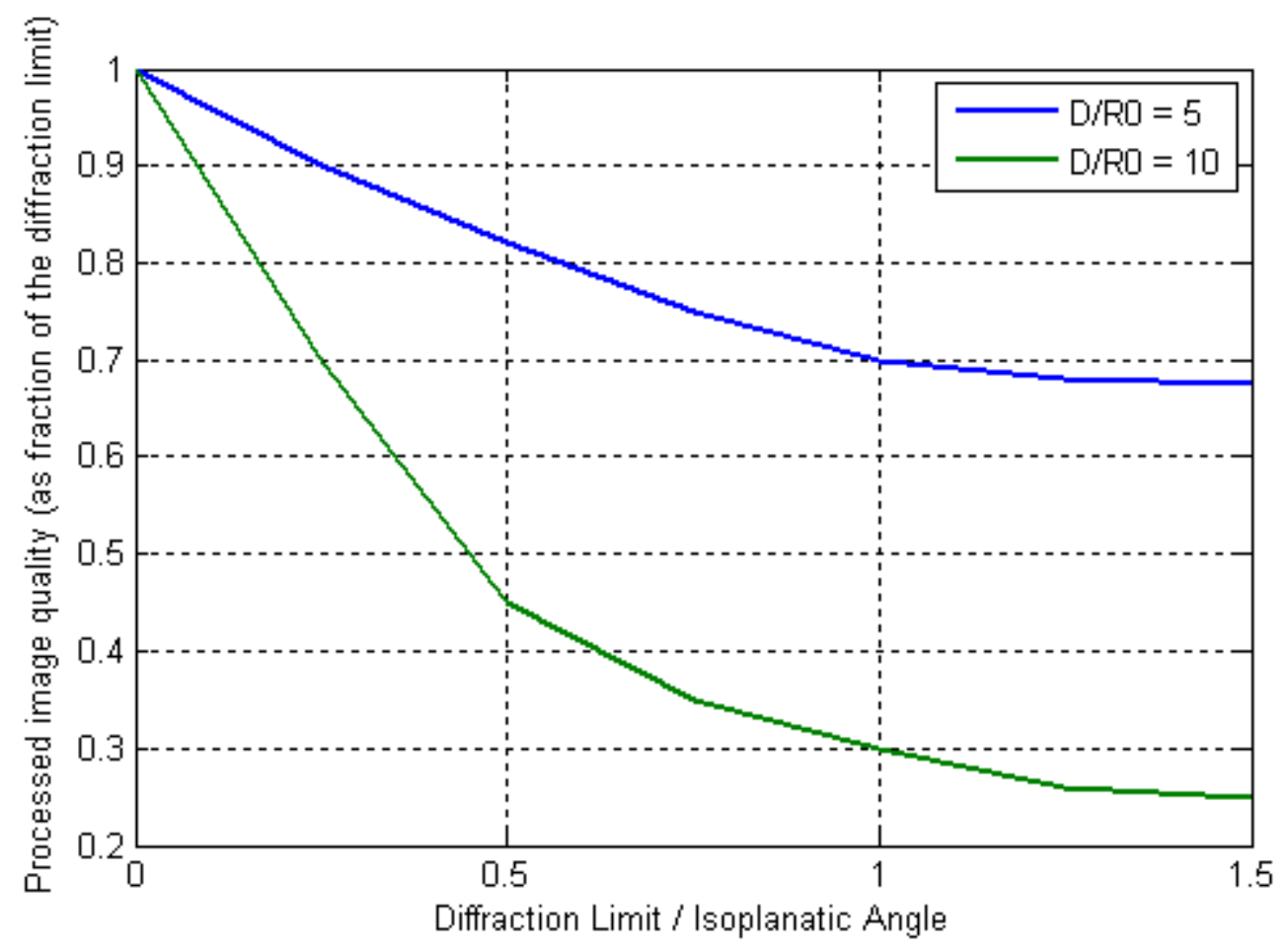

Figure 5: Rough first order effects of isoplanatic angle on speckle processed image quality. 


\subsection{Comparison of real data in this regime with simulation}

In order to validate the simulations that will be shown, we take a look at some real data acquired in a similar regime to the charts. The data shown here was taken in Livermore, CA from a low hillside at $160 \mathrm{~m}$ altitude looking downwards at a $3.3 \mathrm{~km}$ range over foliage and a road on a warm winter day. Our telescope system was a $20 \mathrm{~cm}$ diameter Celestron with a 3x Barlow lens giving an effective focal length of 6 meters. Our QImaging CCD camera had a pixel size of $6.7 \mu \mathrm{m}$. This means that our pixel size on target was $3.7 \mathrm{~mm}$ and since the diffraction limit of the telescope is $8.2 \mathrm{~mm}$ at $3.3 \mathrm{~km}$, we were just better than Nyquist sampled. An example short exposure frame from the 100 frame sequence is shown in Figure 6a. The result of summing all 100 frames together after they are registered is shown in Figure $6 \mathrm{~b}$. The speckle processed result is given in Figure $6 \mathrm{c}$. The $\mathrm{r}_{0}$ estimated from the speckle processing is roughly $2 \mathrm{~cm}$. Given the slant path geometry, that works out to $\mathrm{a}_{\mathrm{n}}{ }^{2}$ of $3.5 \mathrm{e}-12$ at the ground level. This is a very reasonable value for $\mathrm{C}_{\mathrm{n}}{ }^{2}$ and corresponds to a stronger turbulence case considered here. The isoplanatic angle calculation gives a value $2 x$ below the diffraction limit, so with a $\mathrm{D} / \mathrm{r} 0$ of 10 means we would expect a degradation allowing us to clearly see features a $3 x$ to $4 x$ the diffraction limit. Note that the speckle processed image clearly shows features at $\sim 3$ times diffraction limit; for example the fingers and facial features are very apparent, which have $\sim 25 \mathrm{~mm}$ (1 inch) scale.

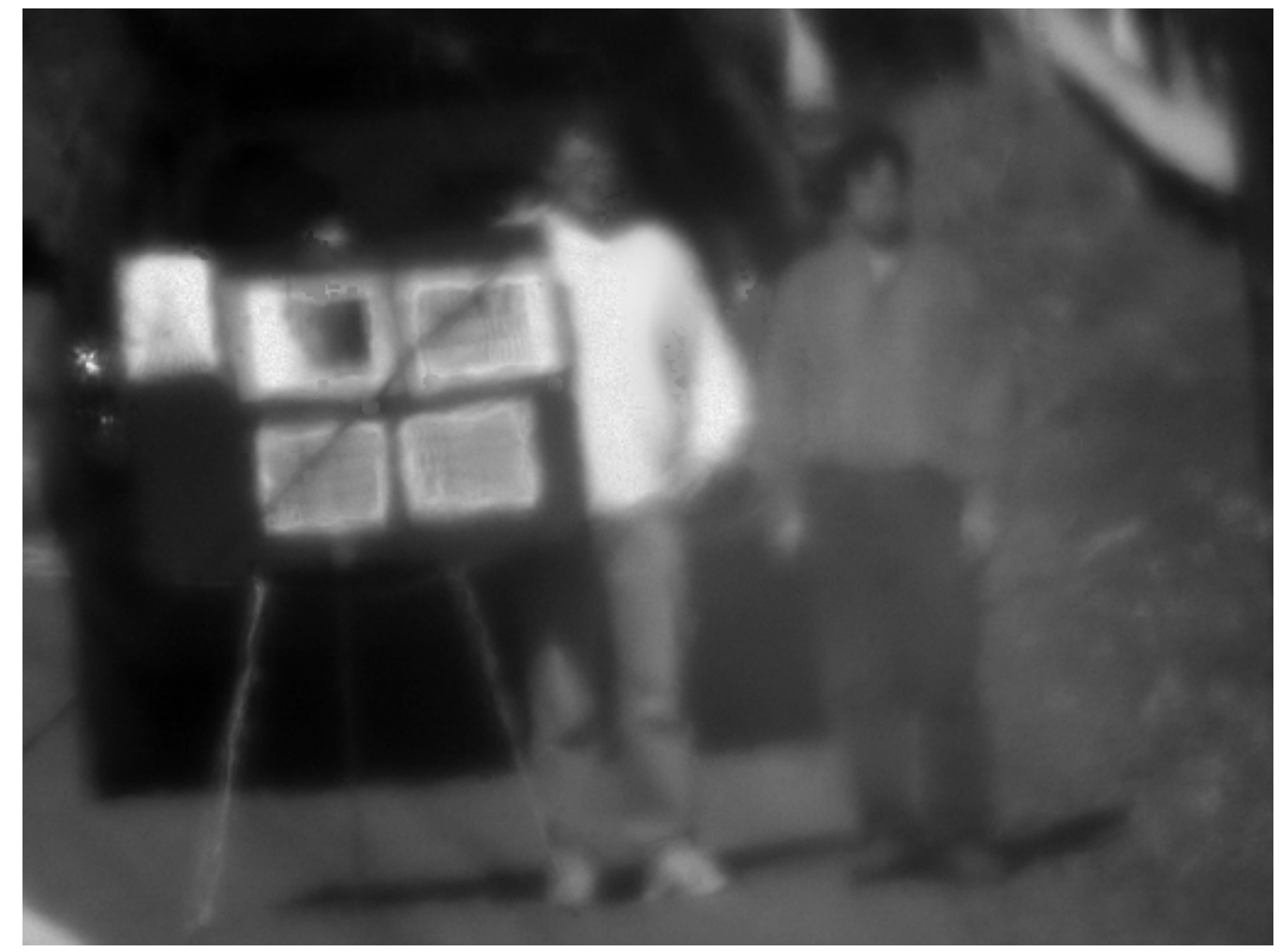

Figure 6a: Sample short exposure frame $(\mathrm{dt}=15 \mathrm{~ms})$ 


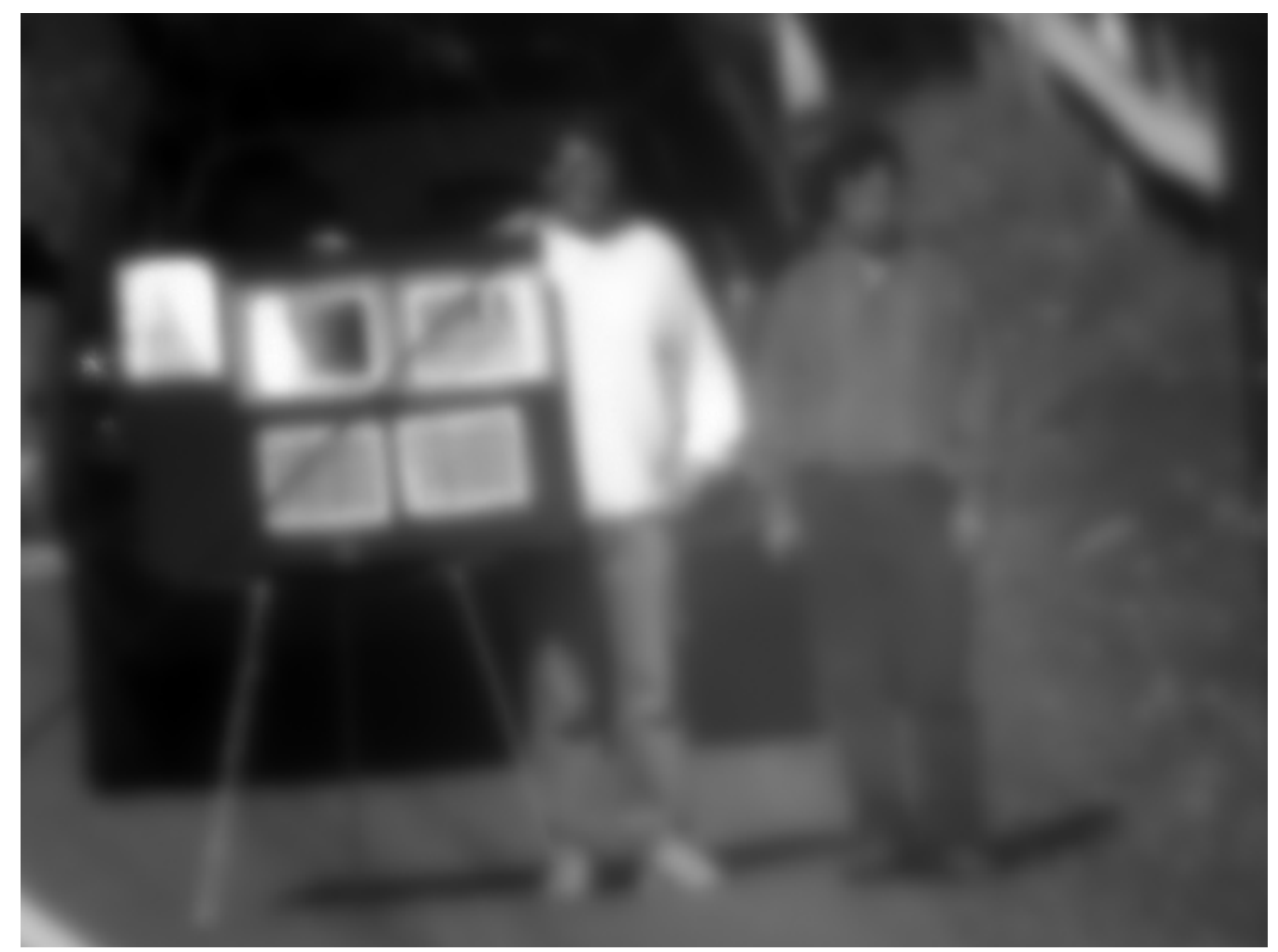

Figure 6b: Shift-and-add.

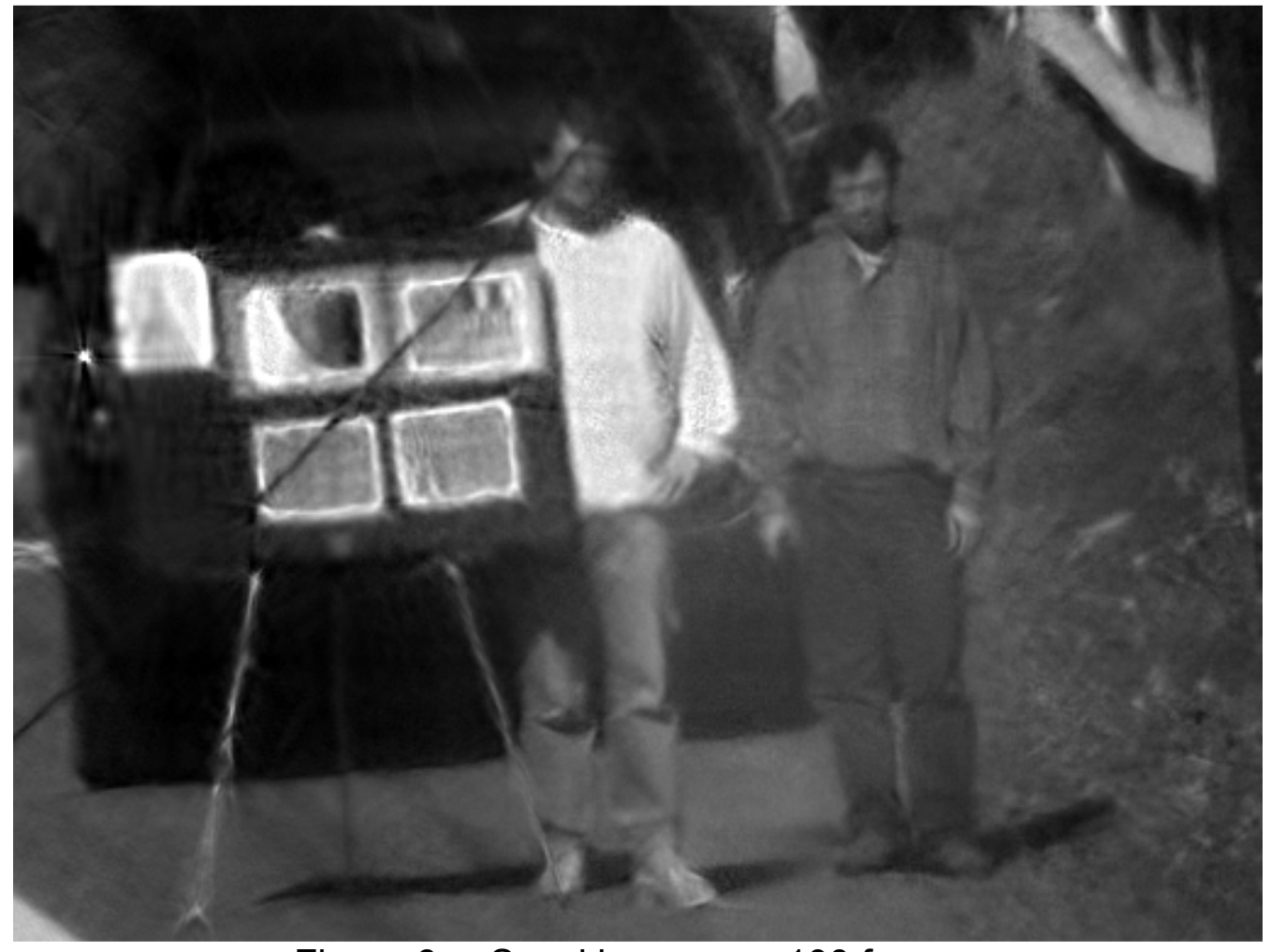

Figure 6c: Speckle process 100 frames 
For the comparison simulation, we consider a USAF test target sampled at Nyquist. See Figure 7. In order to simulate short exposure frames through atmosphere, we generate multiple Kolmogorov phase screens and combine with a circular aperture function to create incoherent point spread functions that we can then convolve with the raw imagery. Using the same $r_{0}$ value estimated from our real data case, a simulated example point spread function and associated raw speckle image is shown in Figure 8a. The shift-and-add result of summing 100 registered simulated frames is shown in Figure $8 \mathrm{~b}$ along with the real data at the same spatial scale. Note that the resolution of the simulated data is very close to it in quality. Speckle processing the 100 frame simulation gives a very nice reconstruction which is shown in Figure 8c. As discussed earlier, the first order effect of anisoplanatism is a reduction in resolution; we include this effect in Figure $8 \mathrm{c}$ by band-limiting the speckle processing in the simulation to a resolution closer to that observed in the real data. We will include contrast and noise effects in the next section.

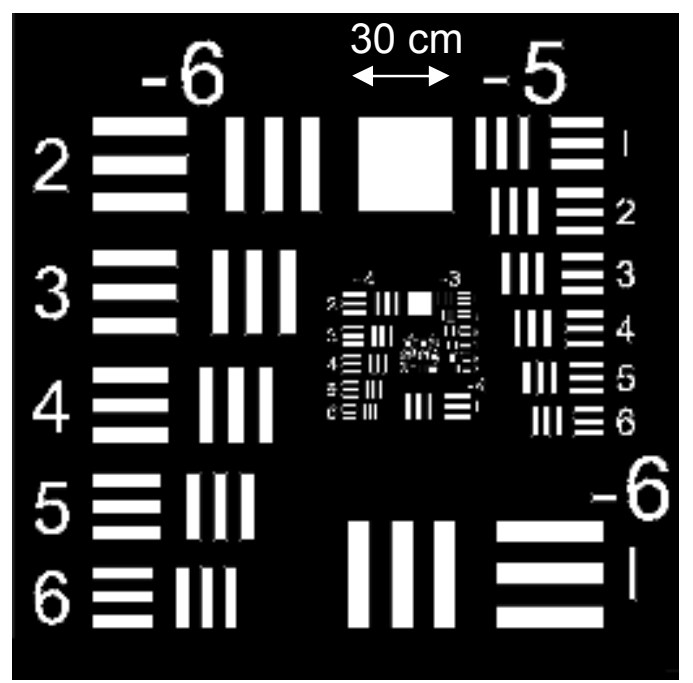

Figure 7: Object being simulated. For the $3.3 \mathrm{~km}$ simulation, the sample interval is taken to be diffraction limit/2 or $4.1 \mathrm{~mm} / \mathrm{pixel}$. The large square on this image would then be $30 \mathrm{~cm}$ across. 


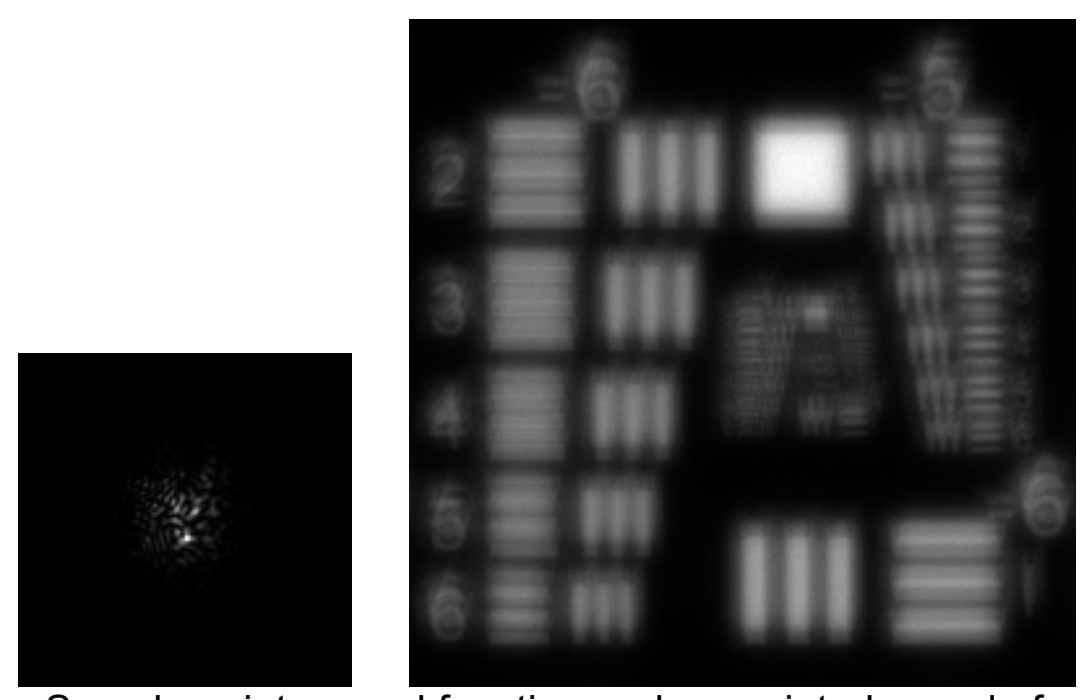

8a: Sample point spread function and associated sample frame
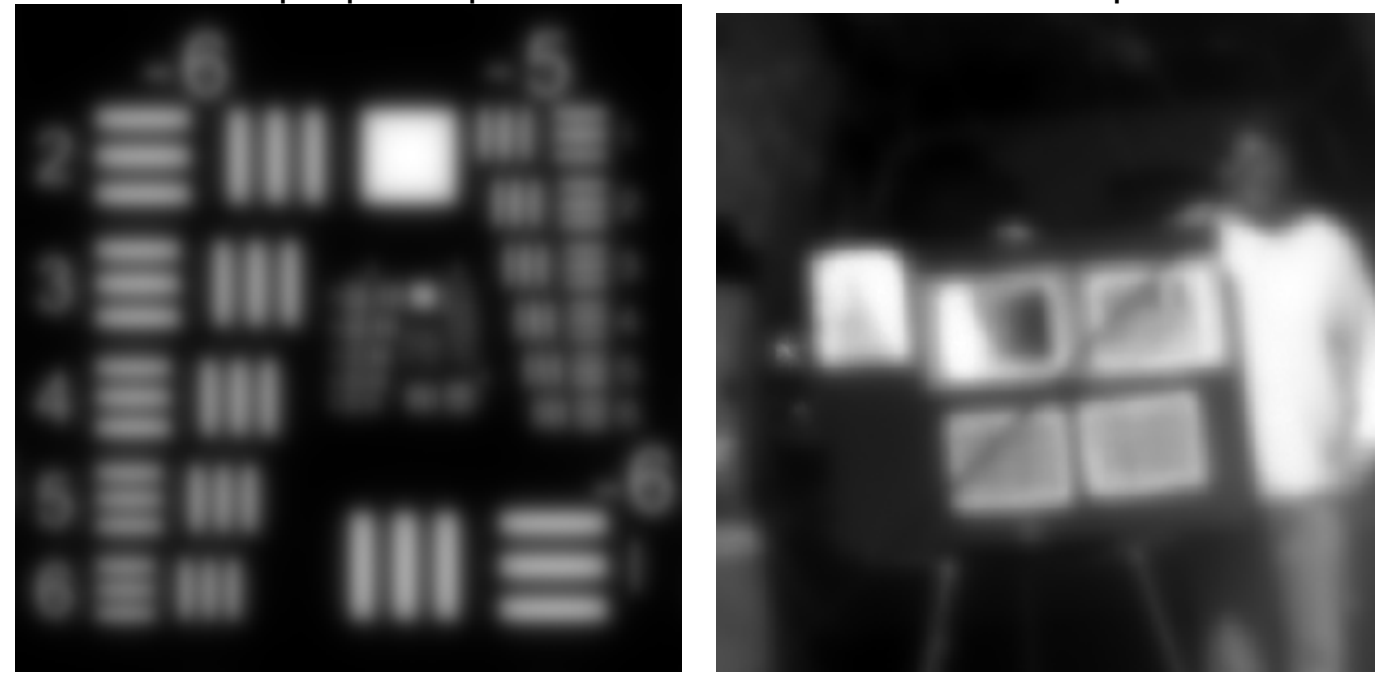

8b: Shift-and-add 100 frames of simulation and real data, same scale
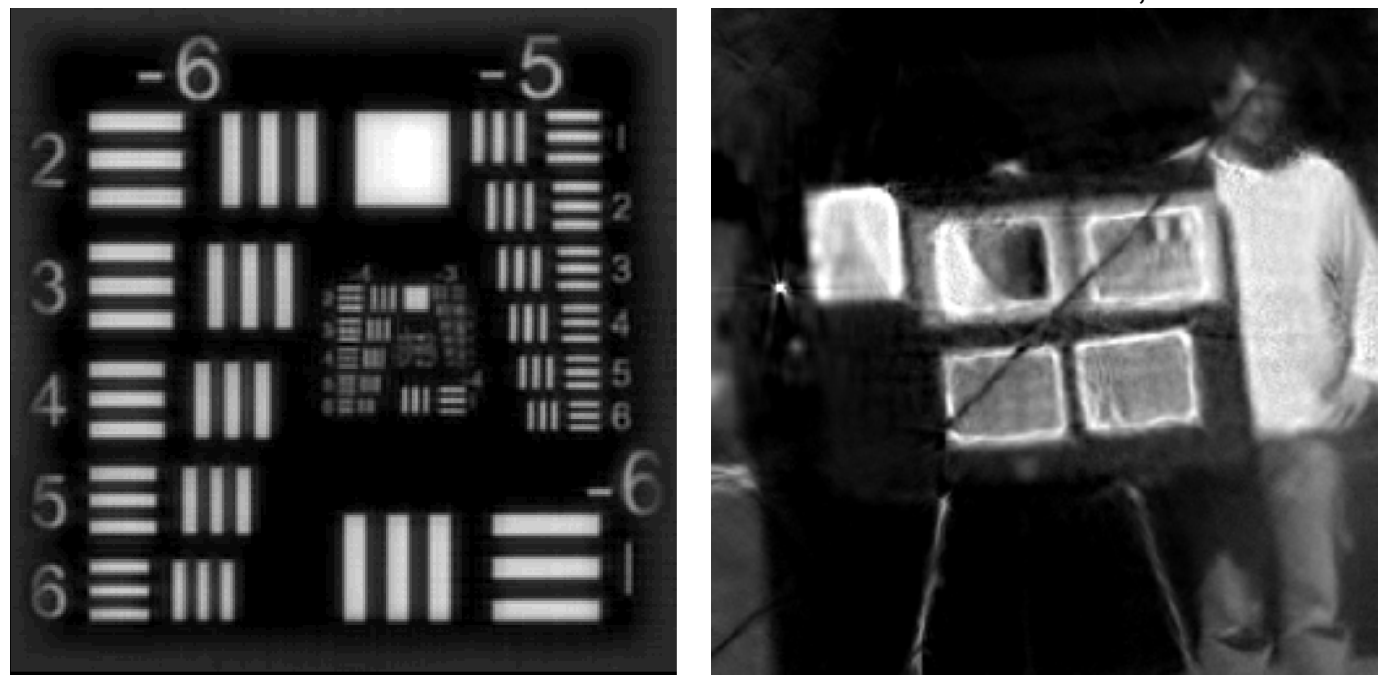

8c: Speckle processed 100 frames of simulation and real data. Lowered the frequency cutoff ( $31 \%$ of max) to simulate first order effect of anisoplanatism to give a resolution close to the real data observations. 


\subsection{Simulated imagery and effect of speckle processing}

In order to get meaningful signal to noise levels given different levels of reflectance or object contrasts, we need to include radiometric effects. Given full solar irradiance $\left(1250 \mathrm{~W} /\left(\mu \mathrm{m}^{*} \mathrm{~m}^{2}\right)\right)$ over a broad wavelength range $(500-800$ $\mathrm{nm}$ ) there is roughly $375 \mathrm{Watts} / \mathrm{m}^{2}$ at sea level. Assuming the sensor is set to be sampling at one half the diffraction limited pixel size, a 60\% efficient CCD looking at a $100 \%$ reflecting object will see roughly $8.8 \times 10^{6}$ photons per second. For a $10 \mathrm{~ms}$ integration time there will be $88 \mathrm{~K}$ photons. For the simulations, we will consider 3 cases of target contrasts ranging from high to low:

\begin{tabular}{|l|l|}
\hline Foreground reflectivity & Background reflectivity \\
\hline $100 \%\left(8.8 \times 10^{6} \mathrm{phot} / \mathrm{sec}\right)$ & $0 \%$ \\
\hline $60 \% \quad\left(5.2 \times 10^{6} \mathrm{phot} / \mathrm{sec}\right)$ & $40 \% \quad\left(3.5 \times 10^{6} \mathrm{phot} / \mathrm{sec}\right)$ \\
\hline $53 \% \quad\left(4.7 \times 10^{6} \mathrm{phot} / \mathrm{sec}\right)$ & $47 \% \quad\left(4.1 \times 10^{6} \mathrm{phot} / \mathrm{sec}\right)$ \\
\hline
\end{tabular}

Table 1: Target reflectivity values under consideration and associated photon flux for half-diffraction-limited sampling assuming a $60 \%$ efficient CCD, visible wavelength.

For each of these target contrasts, we will consider three target ranges $(4 \mathrm{~km}, 6$ $\mathrm{km}$, and $8 \mathrm{~km}$ ) for which there will be differing levels of atmospheric turbulence.

\section{$8 \mathrm{~km}$ case, Medium turbulence $\left(r_{0}=1.5 \mathrm{~cm}\right), D=10 \mathrm{~cm}$}

For these cases, the large square in between and just below the -6 and -5 has dimensions of $1.4 \mathrm{~m} \times 1.4 \mathrm{~m}$. The next smaller square, in between and just below the -4 and -3 has dimensions of $36 \mathrm{~cm}$ by $36 \mathrm{~cm}$. Since the diffraction limit of a $10 \mathrm{~cm}$ aperture at $8 \mathrm{~km}$ is $4 \mathrm{~cm}$, and the isoplanatic angle is well below that at the $\mathrm{mm}$ scale for this case, given that we have a $\mathrm{D} / \mathrm{r} 0$ of 6.6 , we expect no more than a factor of three reduction in diffraction-limited resolution performance. 


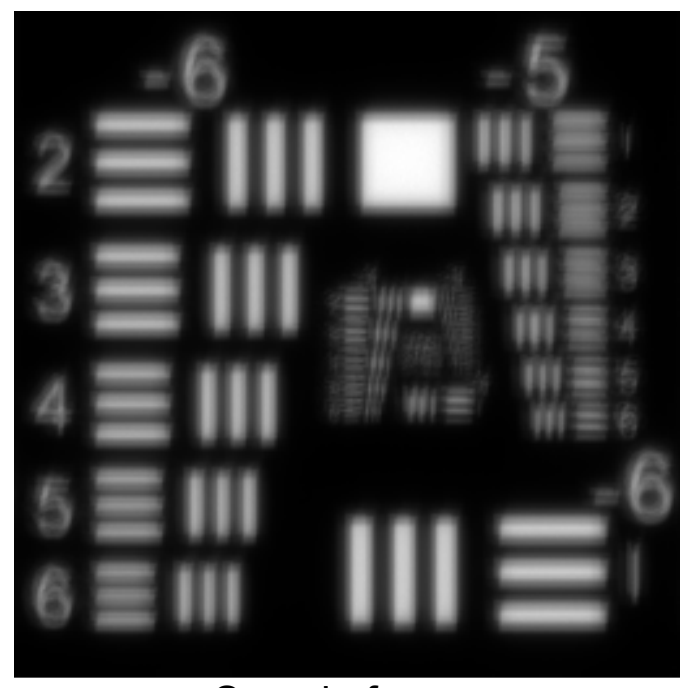

Sample frame

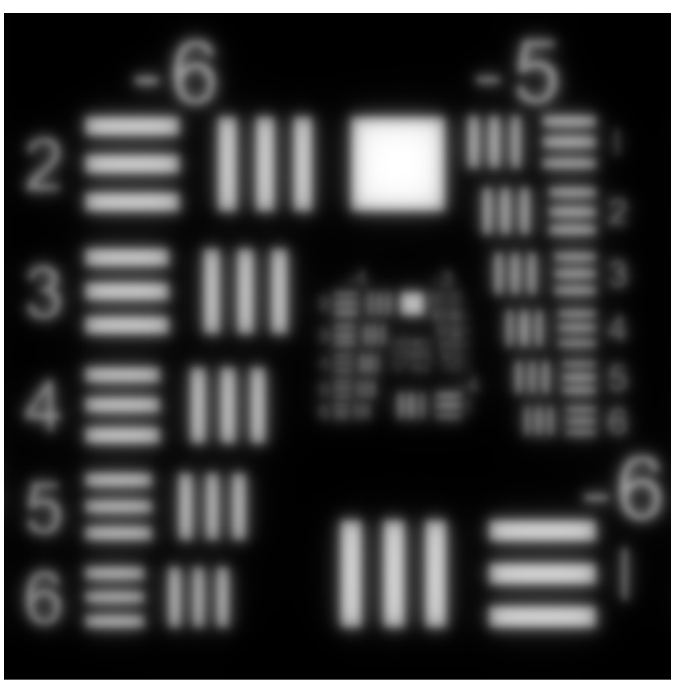

Sum of 100 aligned frames

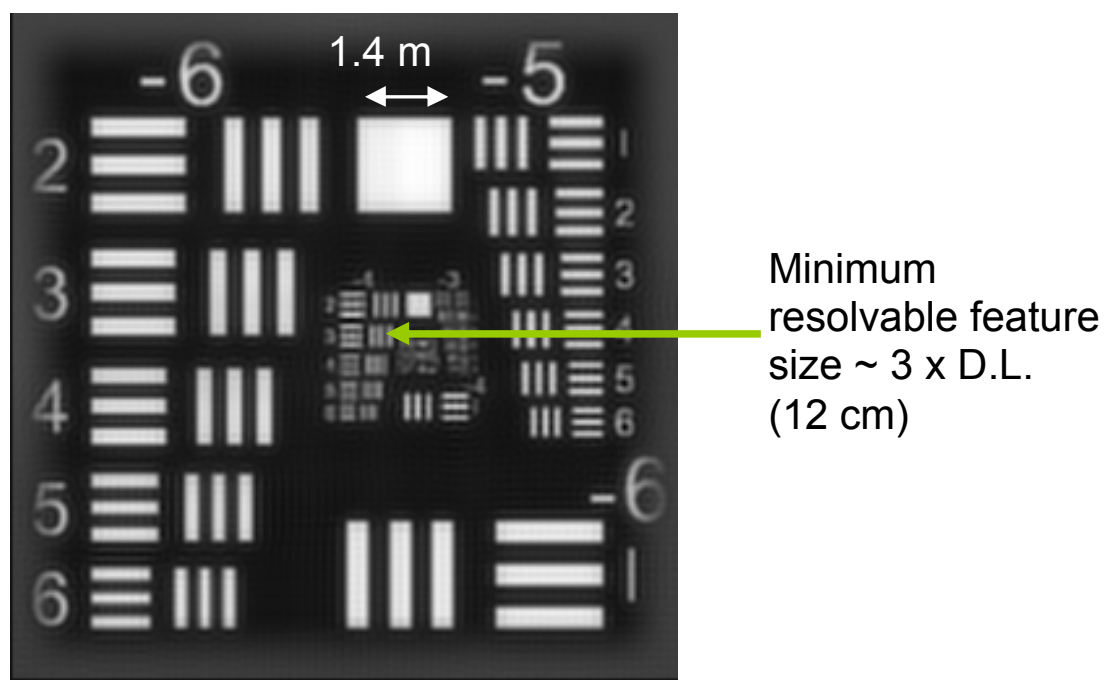

Speckle processed with first order anisoplanatism effect (3x b.w. reduction)

Figure 9a: Target contrast: $100 \% / 0 \%, 8 \mathrm{~km}$ range, medium turbulence, $r_{0}=$ $1.5 \mathrm{~cm}, \mathrm{D}=10 \mathrm{~cm}$. 


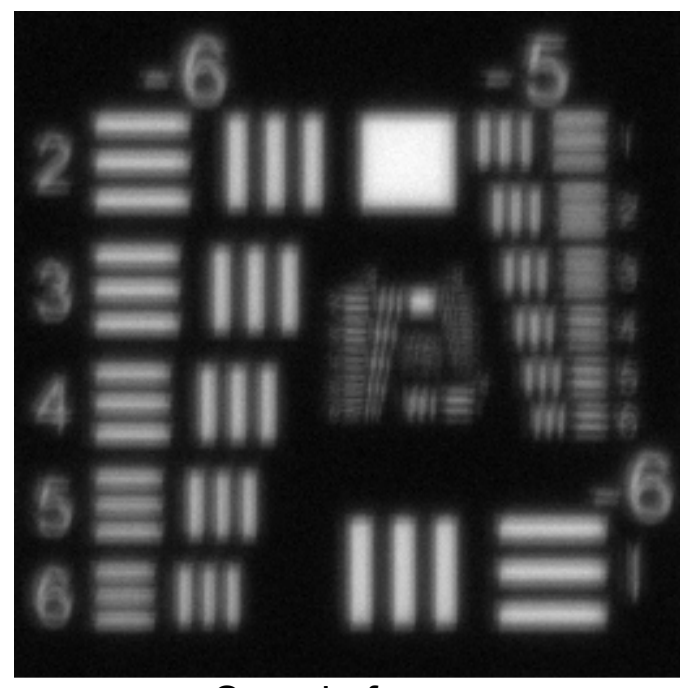

Sample frame

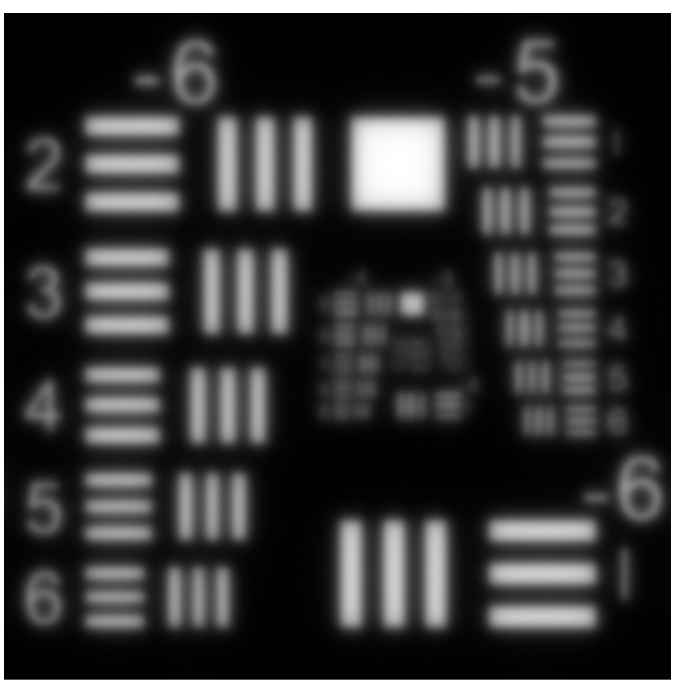

Sum of 100 aligned frames

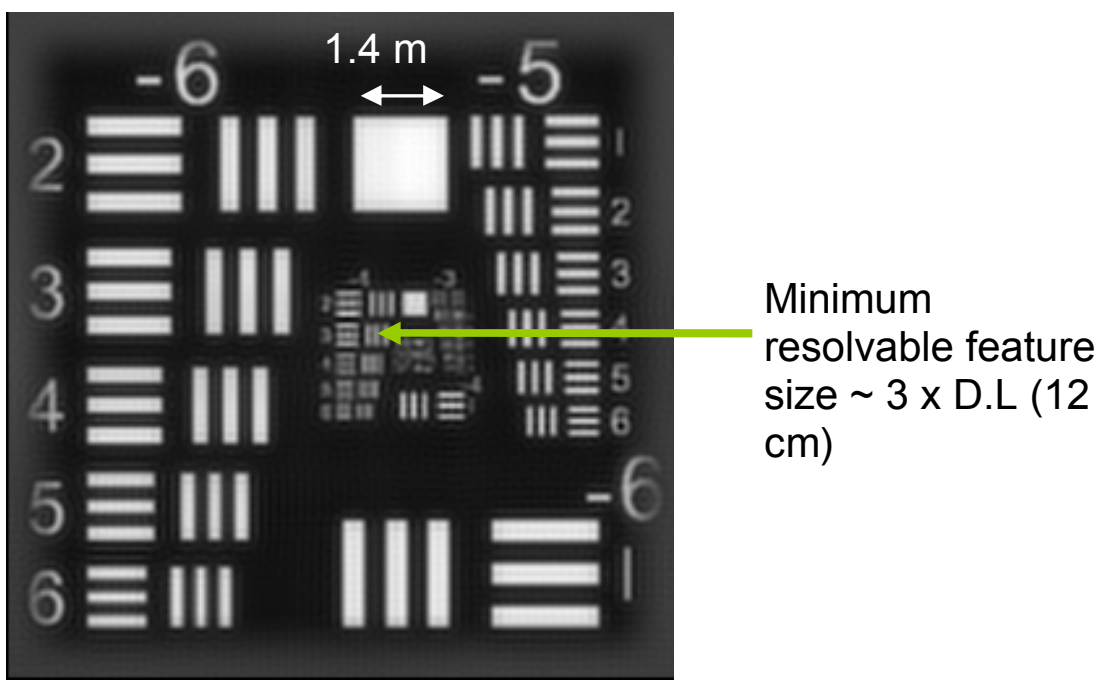

Speckle processed with first order anisoplanatism effect (3x b.w. reduction)

Figure 9b: Target contrast: $60 \% / 40 \%, 8 \mathrm{~km}$ range, medium turbulence, $\mathrm{r}_{0}=$ $1.5 \mathrm{~cm}, \mathrm{D}=10 \mathrm{~cm}$. 


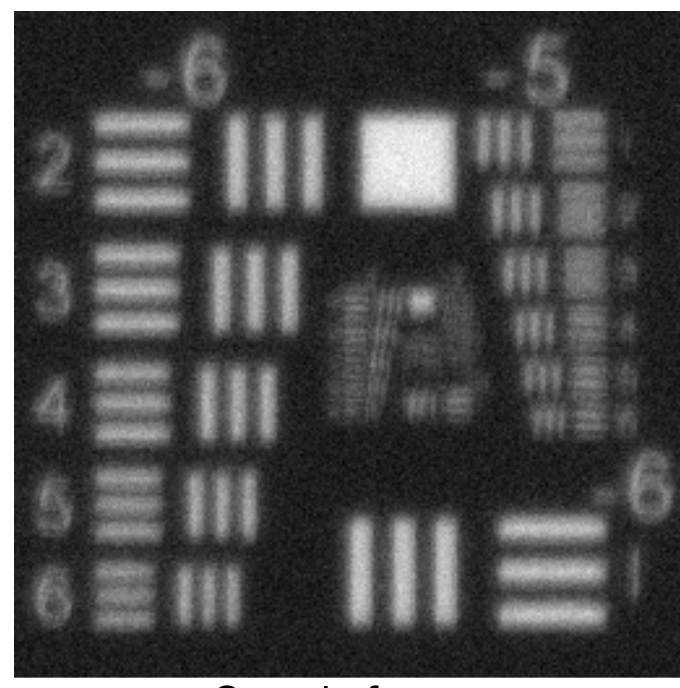

Sample frame

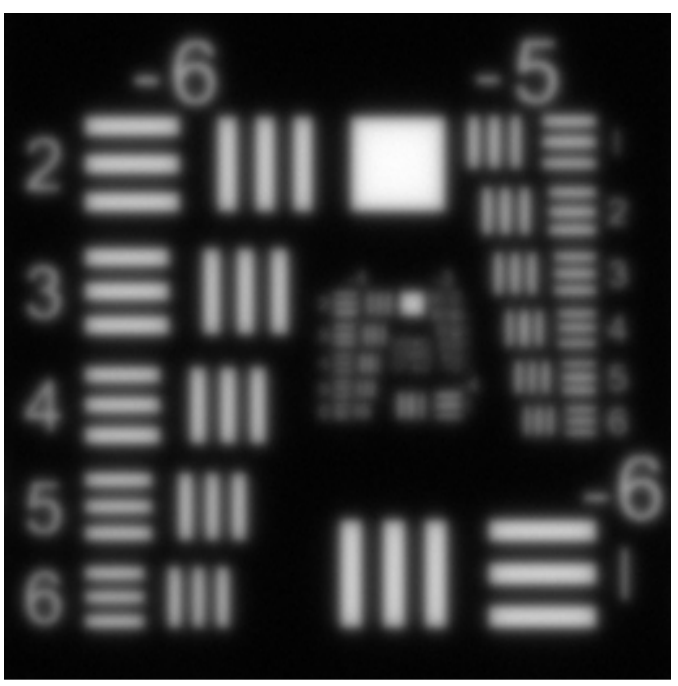

Sum of 100 aligned frames

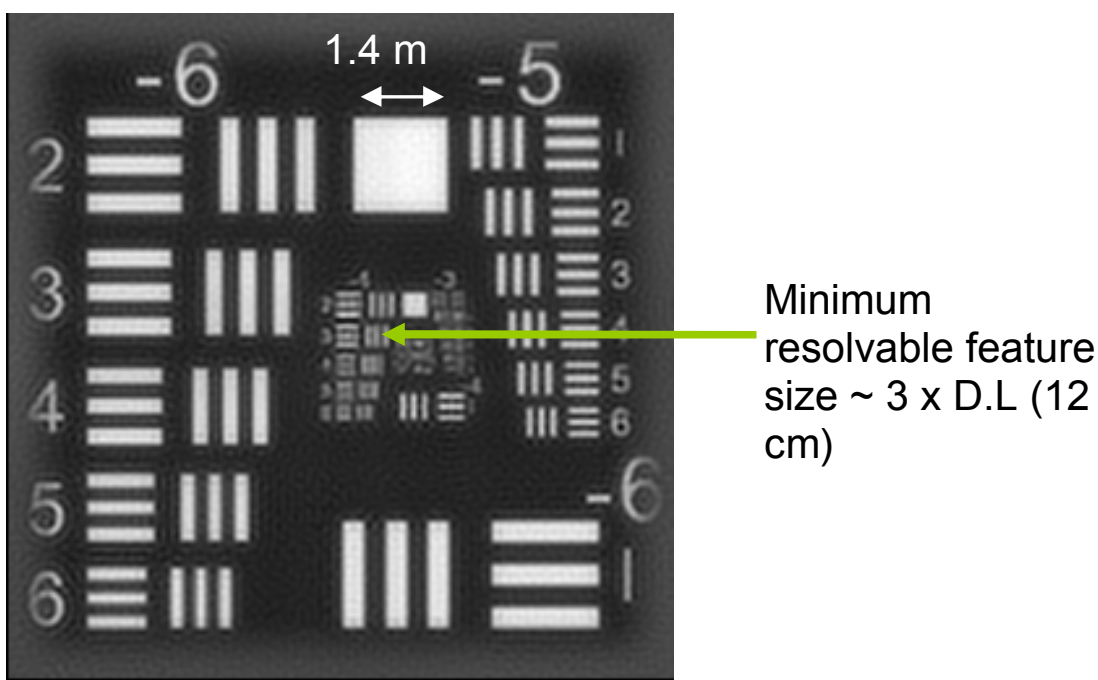

Speckle processed with first order anisoplanatism effect (3x b.w. reduction)

Figure 9c: Target contrast: $53 \%$ / 47\%, medium turbulence, $8 \mathrm{~km}$ range, $\mathrm{r}_{0}=$ $1.5 \mathrm{~cm}, \mathrm{D}=10 \mathrm{~cm}$. 


\section{$6 \mathrm{~km}$ case, Medium turbulence $(\mathrm{r} 0=1.8 \mathrm{~cm}), \mathrm{D}=10 \mathrm{~cm}$}

For these cases, the large square in between and just below the -6 and -5 has dimensions of $107 \mathrm{~cm} \times 107 \mathrm{~cm}$. The next smaller square, in between and just below the -4 and -3 has dimensions of $27 \mathrm{~cm}$ by $27 \mathrm{~cm}$. Since the diffraction limit of a $10 \mathrm{~cm}$ aperture at $6 \mathrm{~km}$ is $3 \mathrm{~cm}$, and the isoplanatic angle is again well below that at the $\mathrm{mm}$ scale for this case, given that we have a $\mathrm{D} / \mathrm{r} 0$ of 5.5 , we expect no more than a factor of three reduction in diffraction-limited resolution performance and in fact may even be slightly better than that.

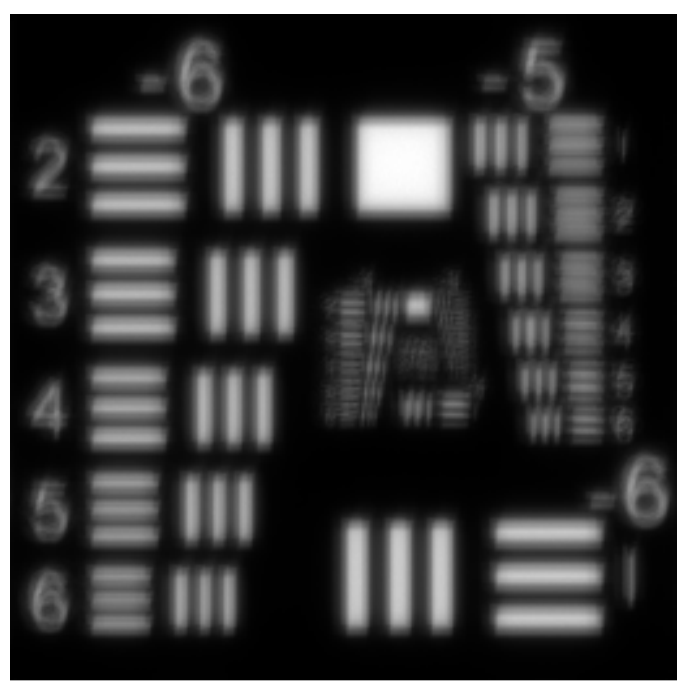

Sample frame

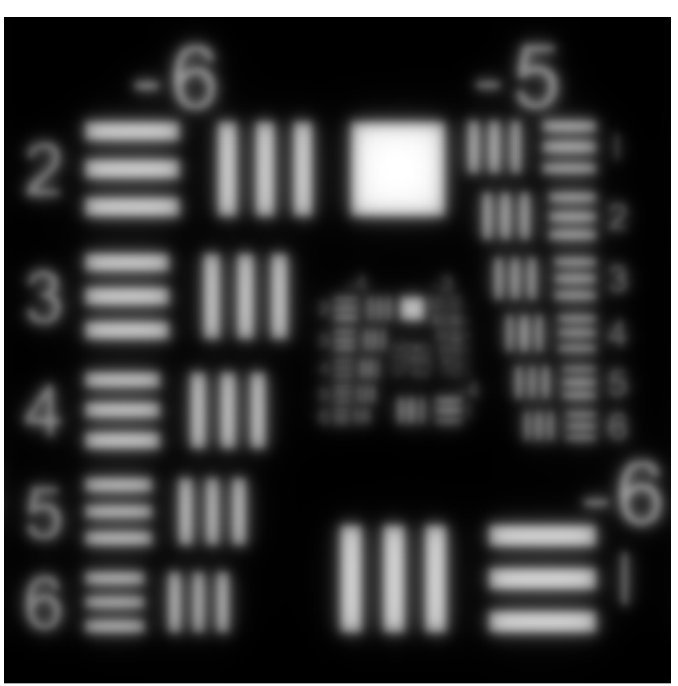

Sum of 100 aligned frames

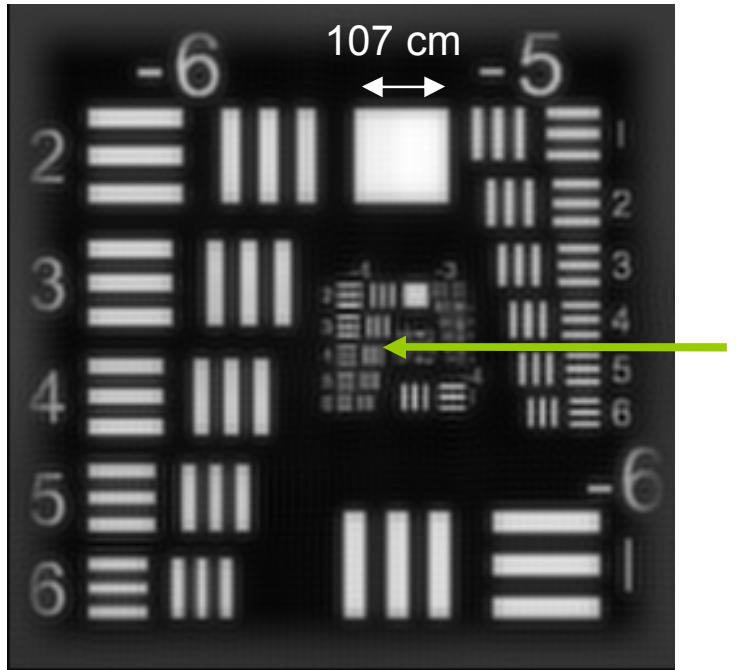

Minimum

resolvable feature size $<\sim 2.5-3 \times$ D.L $(7.5 \mathrm{~cm})$

Speckle processed with first order anisoplanatism effect (3x b.w. reduction)

Figure 10a: Target contrast: $100 \% / 0 \%, 6 \mathrm{~km}$ range, medium turbulence, $\mathrm{r}_{0}=$ $1.8 \mathrm{~cm}, \mathrm{D}=10 \mathrm{~cm}$. 


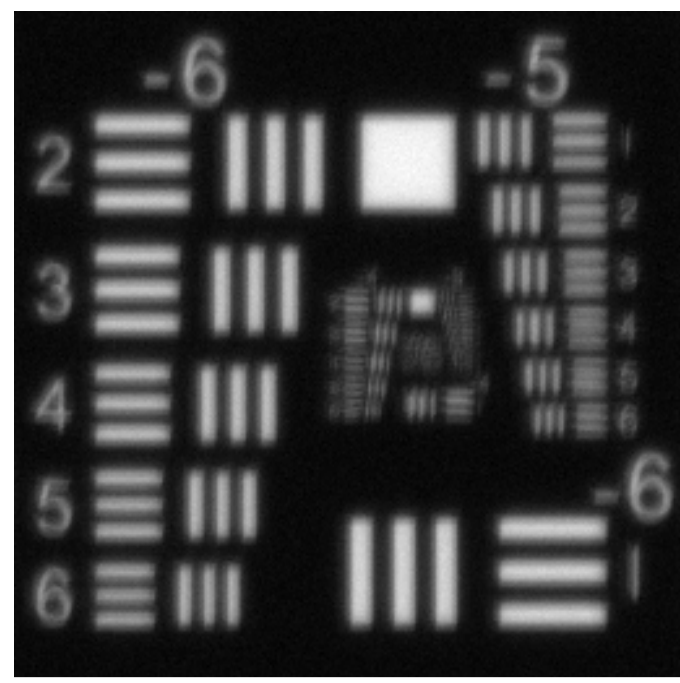

Sample frame

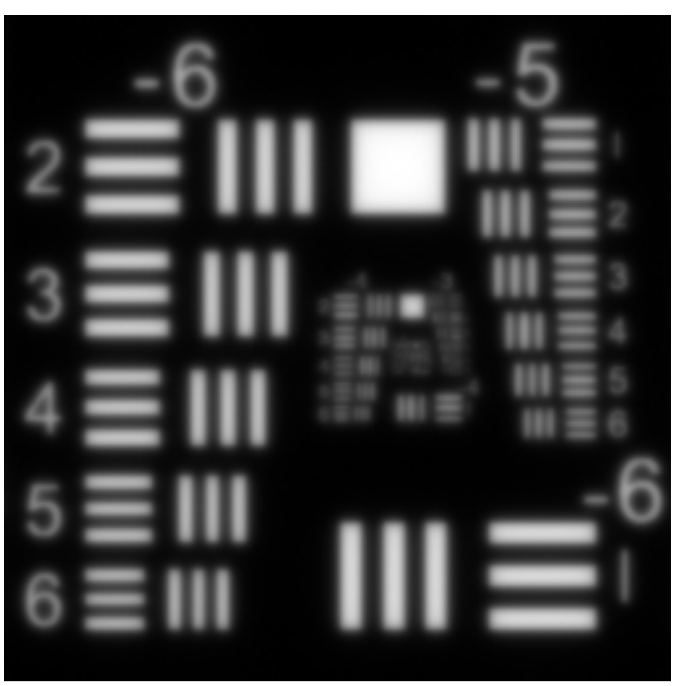

Sum of 100 aligned frames

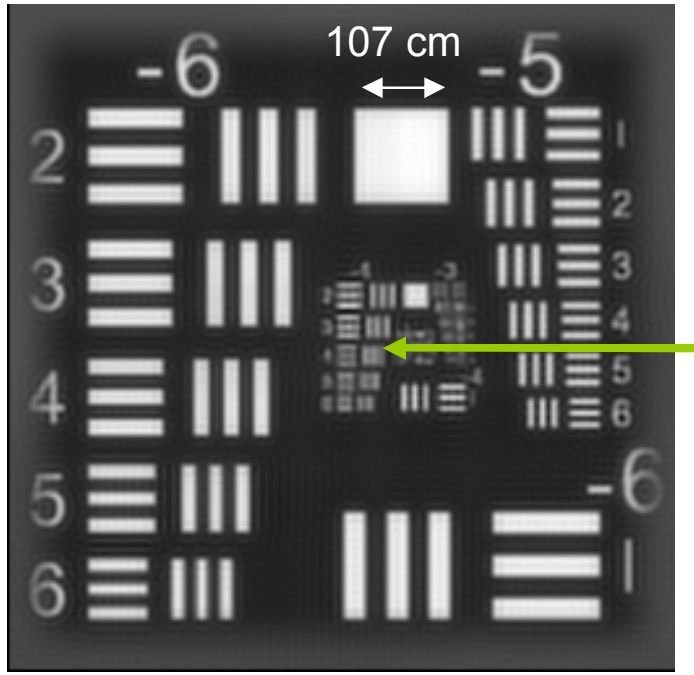

Minimum

resolvable feature size $<\sim 2.5-3 \times$ D.L $(7.5 \mathrm{~cm})$

Speckle processed with first-order anisoplanatism effect (3x b.w. reduction)

Figure 10b: Target contrast: $60 \% / 40 \%, 6 \mathrm{~km}$ range, medium turbulence, $\mathrm{r}_{0}=$ $1.8 \mathrm{~cm}, \mathrm{D}=10 \mathrm{~cm}$. 


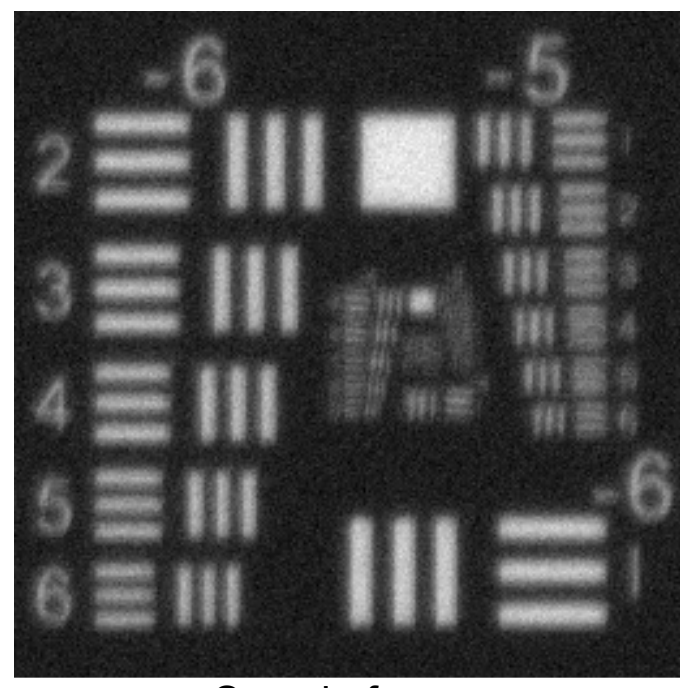

Sample frame

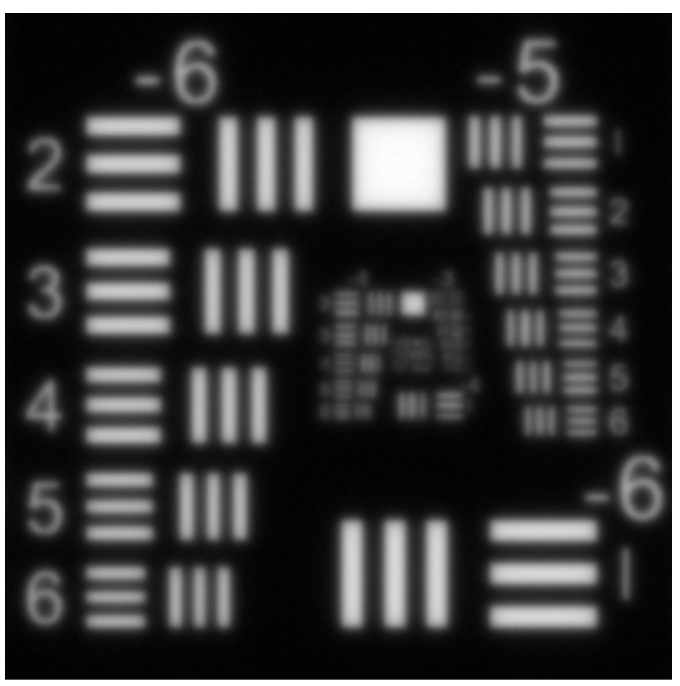

Sum of 100 aligned frames

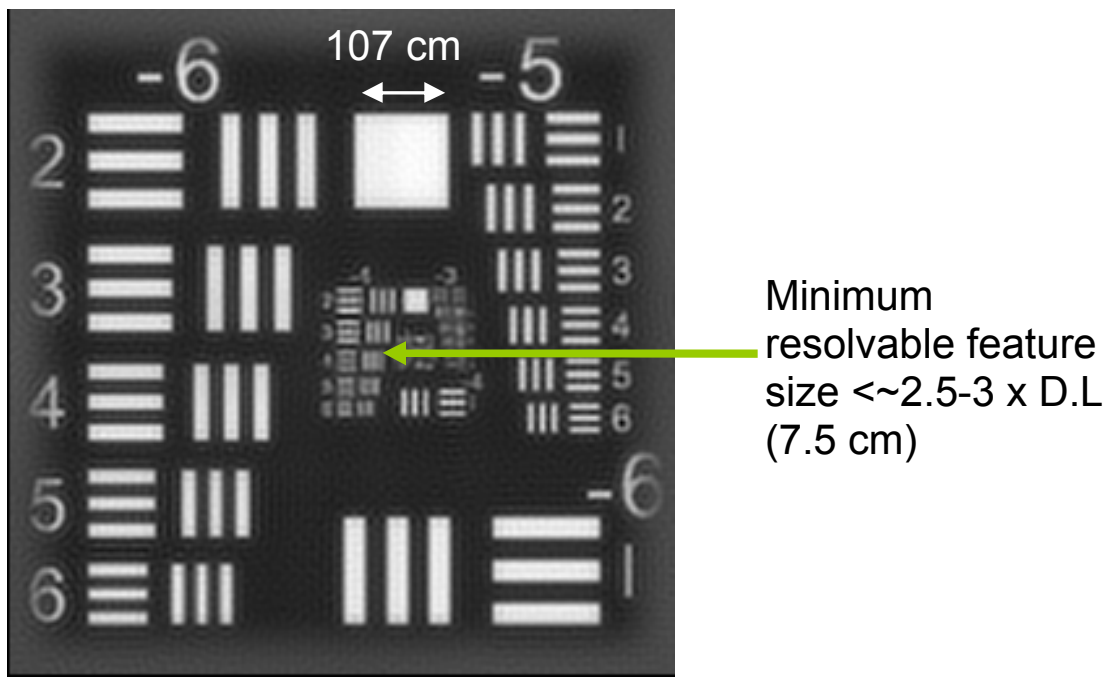

Speckle processed with first-order anisoplanatism effect (3x b.w. reduction)

Figure 10c: Target contrast: $53 \% / 47 \%, 6 \mathrm{~km}$ range, medium turbulence, $r_{0}=$ $1.8 \mathrm{~cm}, \mathrm{D}=10 \mathrm{~cm}$. 


\section{$4 \mathrm{~km}$ case, Medium turbulence $(\mathrm{r} 0=2.2 \mathrm{~cm}), D=10 \mathrm{~cm}$}

For this cases, the large square in between and just below the -6 and -5 has dimensions of $71 \mathrm{~cm} \times 71 \mathrm{~cm}$. The next smaller square, in between and just below the -4 and -3 has dimensions of $18 \mathrm{~cm}$ by $18 \mathrm{~cm}$. The imagery looks very much that of the previous two figure sets. We show the low contrast case in Figure 11. Since the diffraction limit of a $10 \mathrm{~cm}$ aperture at $4 \mathrm{~km}$ is $2 \mathrm{~cm}$, and the isoplanatic angle is again well below that at the $\mathrm{mm}$ scale for this case, given that we have a $\mathrm{D} / \mathrm{r} 0$ of 4.5 , we expect no more than a factor of 1.5 reduction in diffraction-limited resolution performance. This is not even noticeable here.

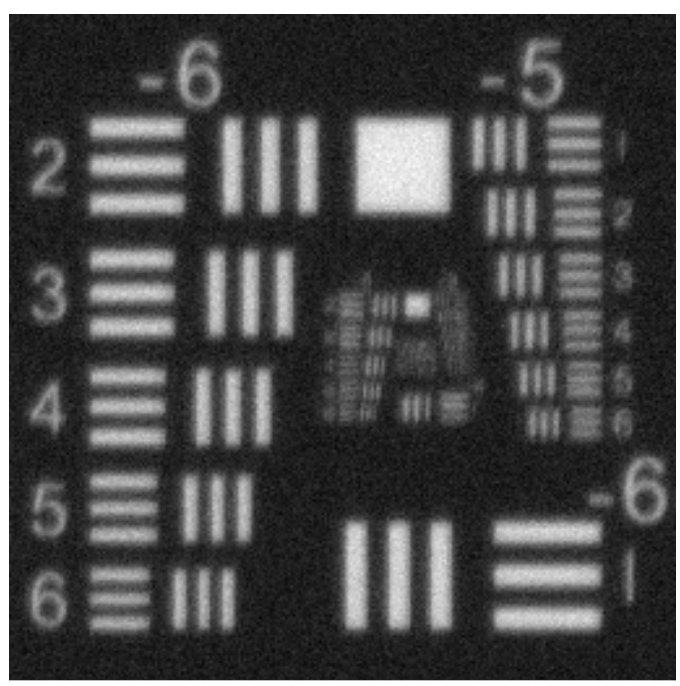

Sample frame

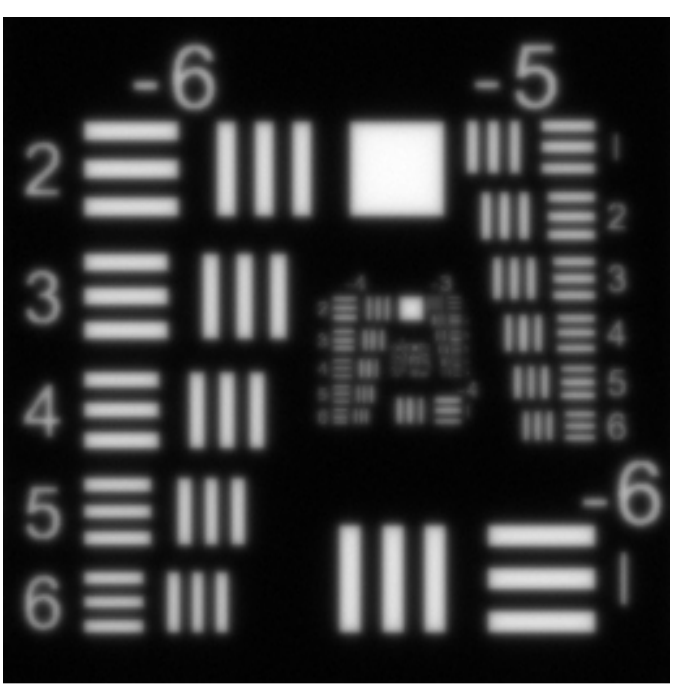

Sum of 100 aligned frames

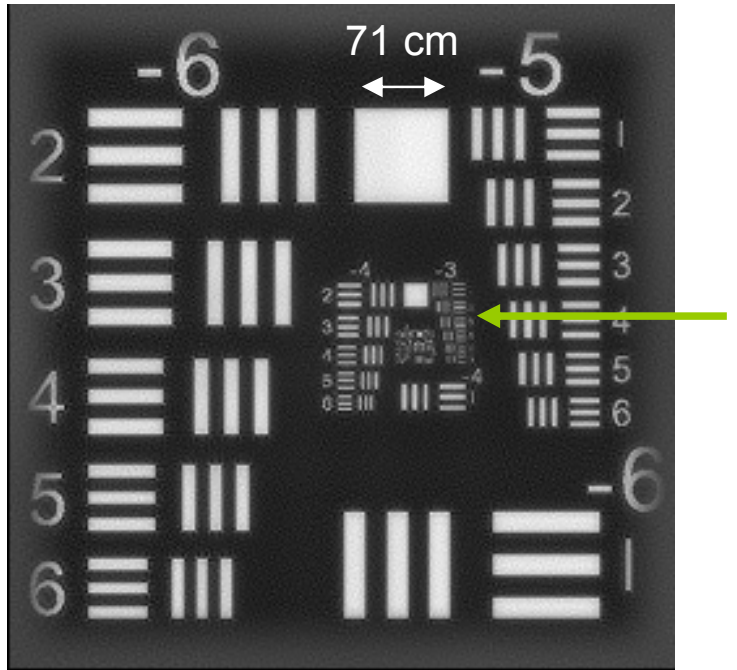

Minimum

resolvable feature size $\sim 1.5 \times$ D.L $(3$ $\mathrm{cm})$

Speckle processed with first-order anisoplanatism effect (30\% b.w. reduction)

Figure 11: Target contrast: $53 \% / 47 \%, 4 \mathrm{~km}$ range, medium turbulence, $r_{0}=$ $2.2 \mathrm{~cm}, \mathrm{D}=10 \mathrm{~cm}$. 
To complete the atmospheric effects section, we now show the strong turbulence case for the two lower low contrast target cases at $6 \mathrm{~km}$ range in Figure 12.

\section{$6 \mathrm{~km}$ case, Strong turbulence $(\mathrm{r} 0=0.45 \mathrm{~cm}), \mathrm{D}=10 \mathrm{~cm}$}

Just as before, since the diffraction limit of a $10 \mathrm{~cm}$ aperture at $6 \mathrm{~km}$ is $3 \mathrm{~cm}$, and the isoplanatic angle is again well below that at the $\mathrm{mm}$ scale for this case, given that we have a $\mathrm{D} / \mathrm{r} 0$ of 22 , although not specifically covered on the chart in Figure 4 , we would expect a larger reduction in diffraction-limited resolution performance in this case, believably by a factor of 10 .

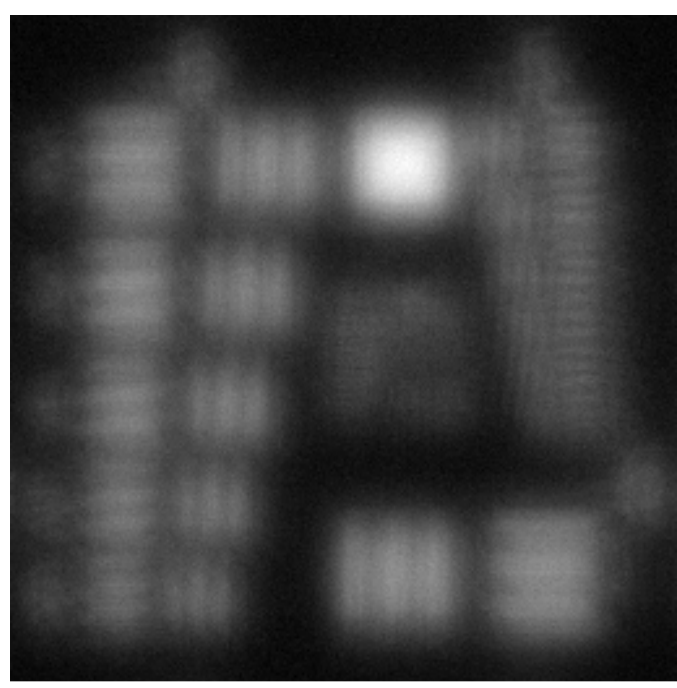

Sample frame

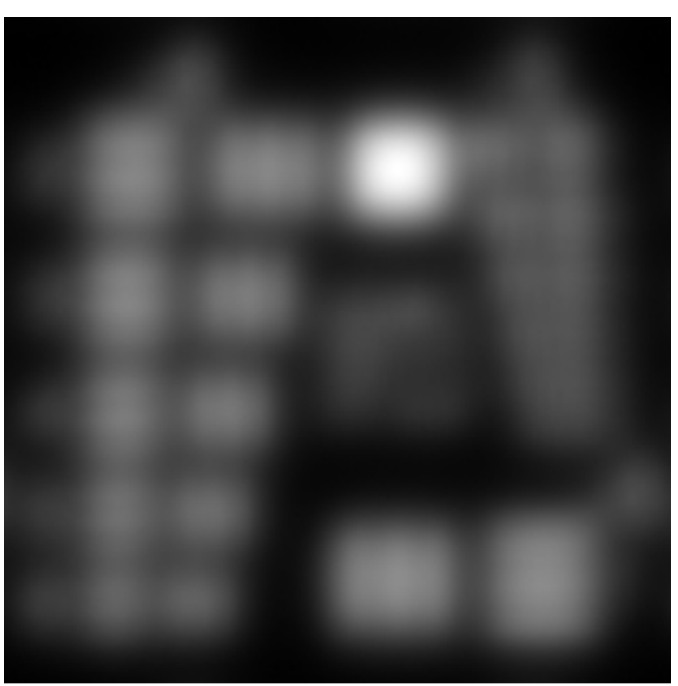

Sum of 100 aligned frames

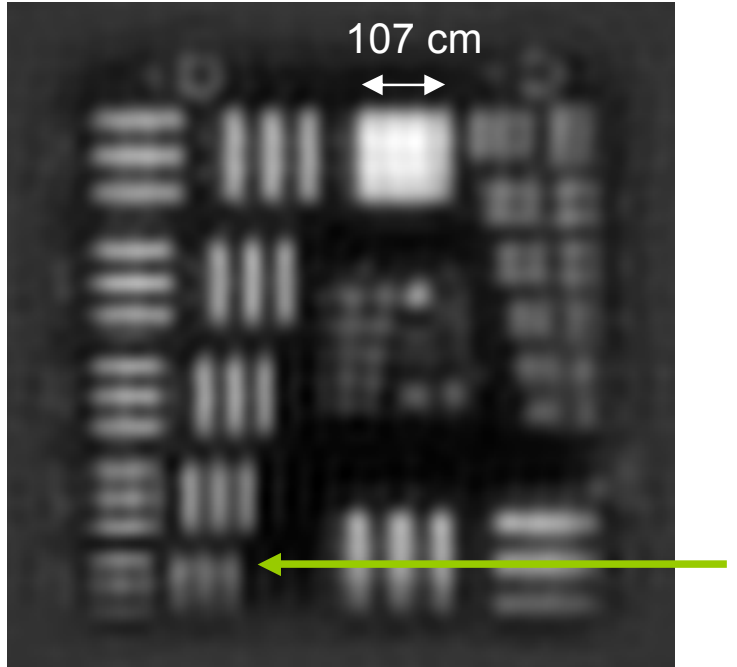

Minimum

resolvable feature

size $\sim 10 \times$ D.L

$(30 \mathrm{~cm})$

Speckle processed with first-order anisoplanatism effect (10x b.w. reduction)

Figure 12a: Target contrast: $60 \% / 40 \%, 6 \mathrm{~km}$ range, strong turbulence, $\mathrm{r}_{0}=$ $0.45 \mathrm{~cm}, \mathrm{D}=10 \mathrm{~cm}$. 


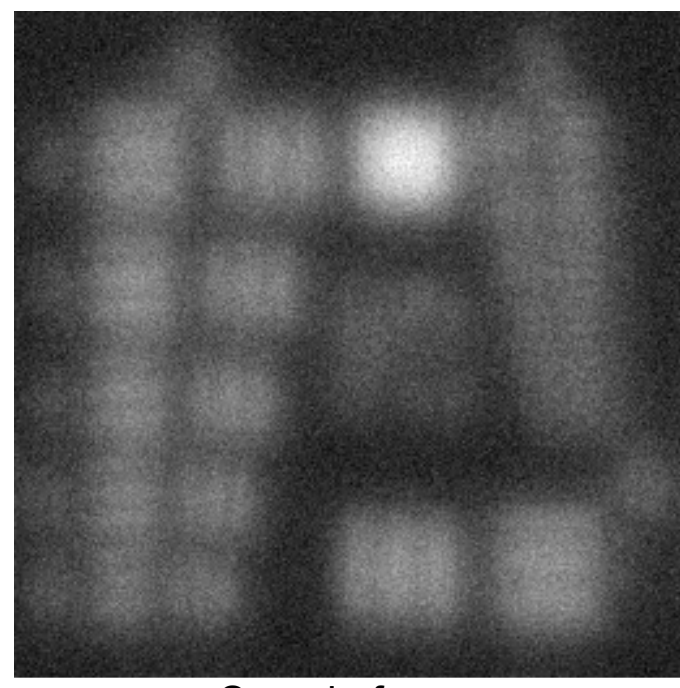

Sample frame

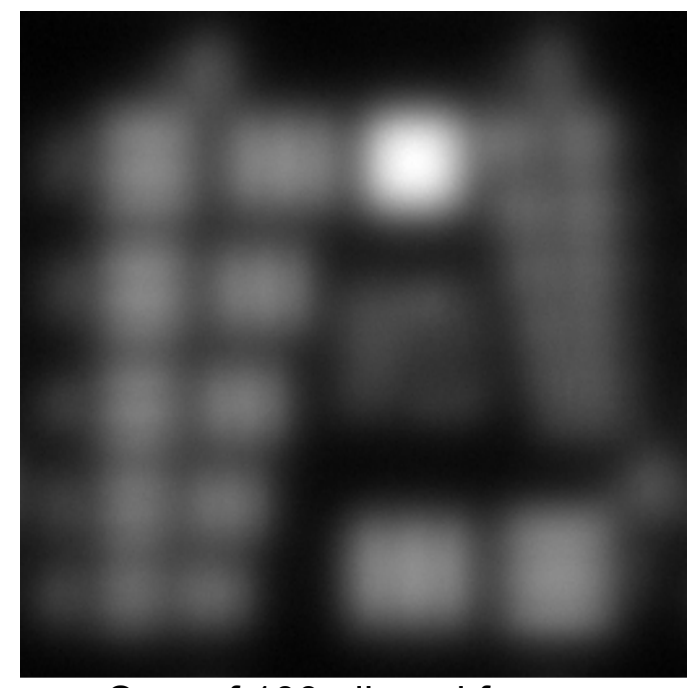

Sum of 100 aligned frames

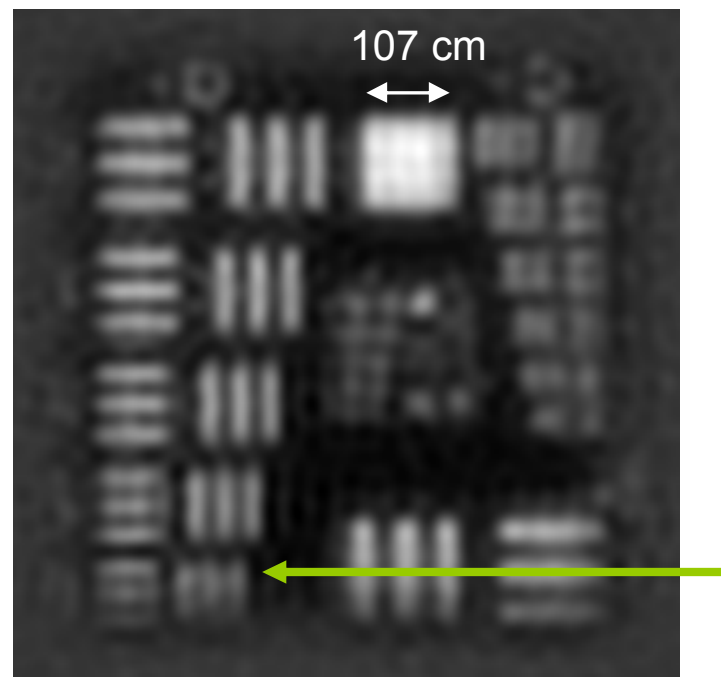

Minimum

resolvable feature size $\sim 10 \times$ D.L $(30 \mathrm{~cm})$

Speckle processed with first-order anisoplanatism effect

Figure 12b: Target contrast: $53 \% / 47 \%, 6 \mathrm{~km}$ range, $\mathrm{r}_{0}=0.45 \mathrm{~cm}, \mathrm{D}=10 \mathrm{~cm}$. We have reduced the bandwidth to $10 \%$ of the diffraction limit for the anisoplanatism effect. 


\subsection{Scenario 2}

For the second scenario, we are also interested in low slant angles from mountains or hillsides. Targets of interest include identifying vehicles and perhaps identifying the cargo being carried on certain vehicles.

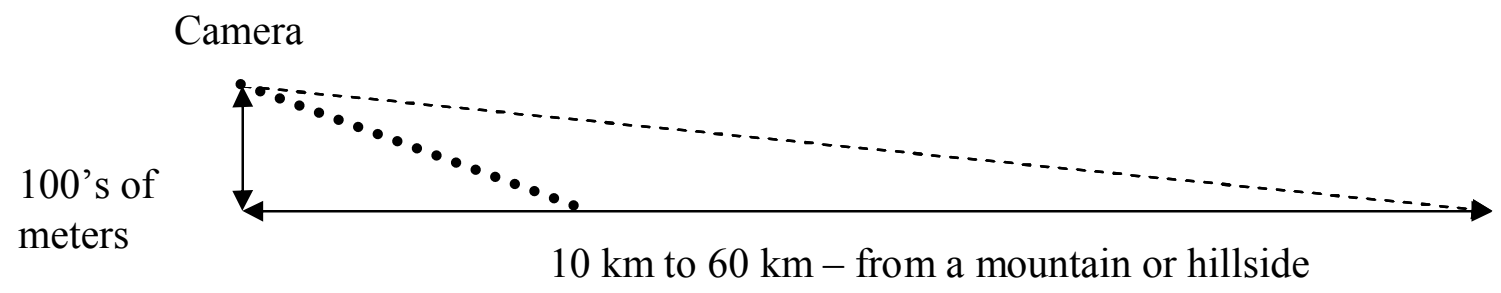

Figure 13: Slant angle imaging geometry. The dashed line is the imaging path above ground. The dotted line represents the mountainside, which is important for knowing the height above ground for the $\mathrm{Cn} 2$ calculations.

\subsection{Aperture alone - the diffraction limit}

Let us first look purely at the diffraction limit of various sized apertures for the ranges of interest. The plot in Figure 14 represents the best that the optical system could ever do, given that the camera and optics are appropriately sampled with sufficient light levels and target contrast. If the requirement was to resolve a $10 \mathrm{~cm}$ sized feature at a distance of $30 \mathrm{~km}$, an optic with a $15 \mathrm{~cm}$ diameter would be needed.

\subsection{Focal length and camera pixel size}

We show the same analysis as in Section 1, but for longer range here in Figure 15. 


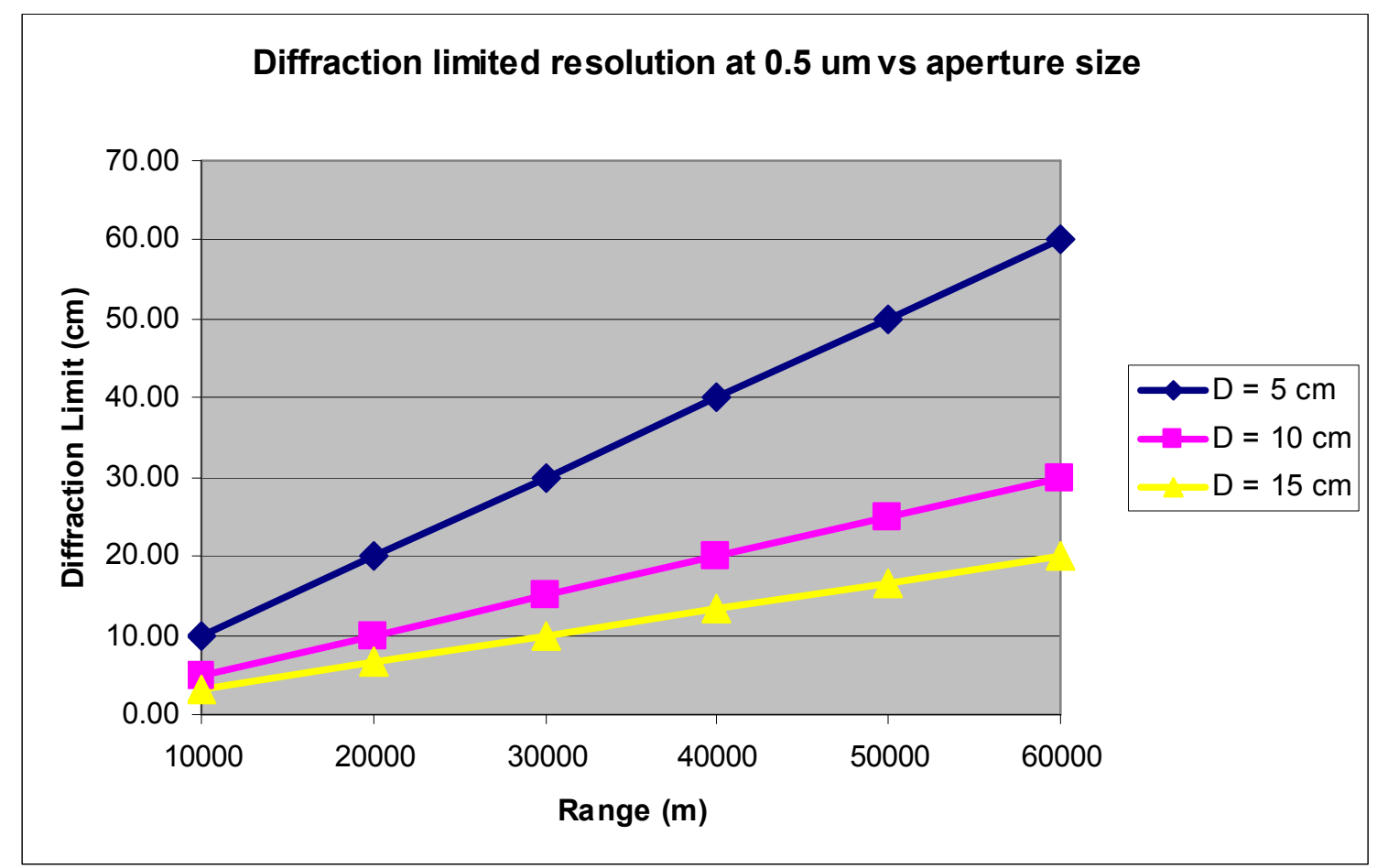

Figure 14: Plot of diffraction limited resolution of three aperture sizes versus range in the visible $(\lambda=0.5 \mu \mathrm{m})$. We calculate this using the formula $(\lambda *$ Range $) / D$

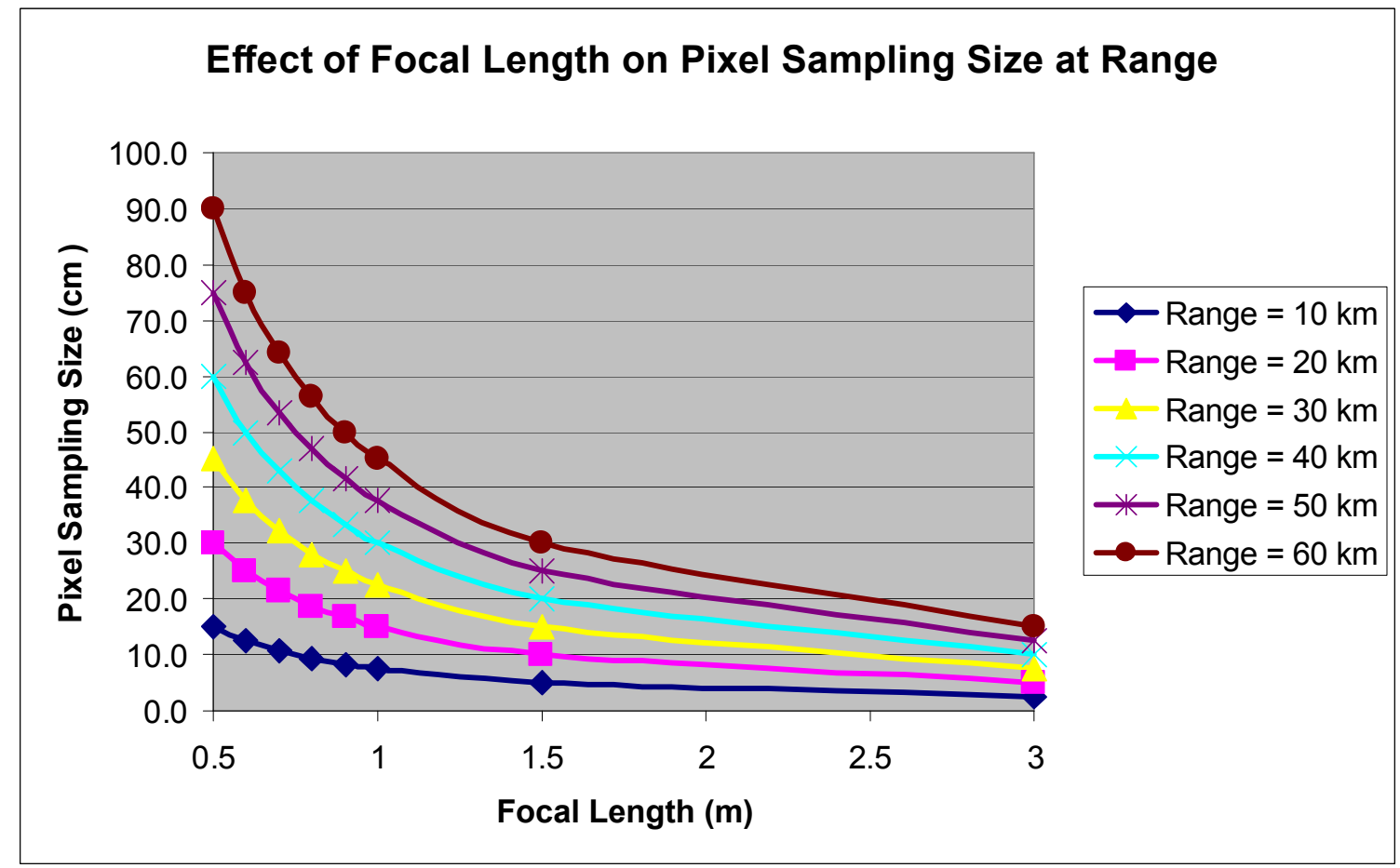

Figure 15: Plot of the pixel size in $\mathrm{cm}$ at range versus focal length and range for a camera with CCD pixel size of $7.5 \mu \mathrm{m}$ per pixel. We calculate this using the formula (ccd pixel size * range / focal length). 


\subsection{Atmospheric effects}

For this scenario, we used the NVESD Kopeika1 $\mathrm{C}_{\mathrm{n}}{ }^{2}$ model to predict $\mathrm{C}_{\mathrm{n}}{ }^{2}$ at the ground layer for three different cases. The strong turbulence case is for a hot windy day in the afternoon $\left(\mathrm{C}_{\mathrm{n}}{ }^{2}=3.54 \mathrm{e}-12 \mathrm{~m}^{-2 / 3}\right.$ at ground level). The medium turbulence case is for late morning on a nice, breezy day with $40 \%$ humidity $\left(C_{n}{ }^{2}=1.0 \mathrm{e}-12 \mathrm{~m}^{-2 / 3}\right.$ at ground level). And the good seeing case is at sunset on a nice day with low wind and $20 \%$ humidity $\left(\mathrm{C}_{\mathrm{n}}{ }^{2}=4 \mathrm{e}-13 \mathrm{~m}^{-2 / 3}\right.$ at ground level). In order to obtain realistic atmospheric values from a mountain top, it is necessary to model the imaging path above the ground properly. For this we assume as certain radius of the base of a mountain such that the mountainside falls away at some angle from the camera. The actual height of the imaging path above the ground is used in the $\mathrm{C}_{\mathrm{n}}{ }^{2}(\mathrm{~h})$ calculations. This model allows us to include the local atmospheric effects close to the telescope.

For these simulations, we assume a camera altitude of 500 meters with a mountain base radius of $2 \mathrm{~km}$ and we vary the range. See Figure $16 \mathrm{a}$ for an example altitude profile and Figure $16 \mathrm{~b}$ for the $\mathrm{r}_{0}$ predictions. Figure 17 shows the expected image degradation based on the $r_{0}$ values for various aperture sizes versus range.

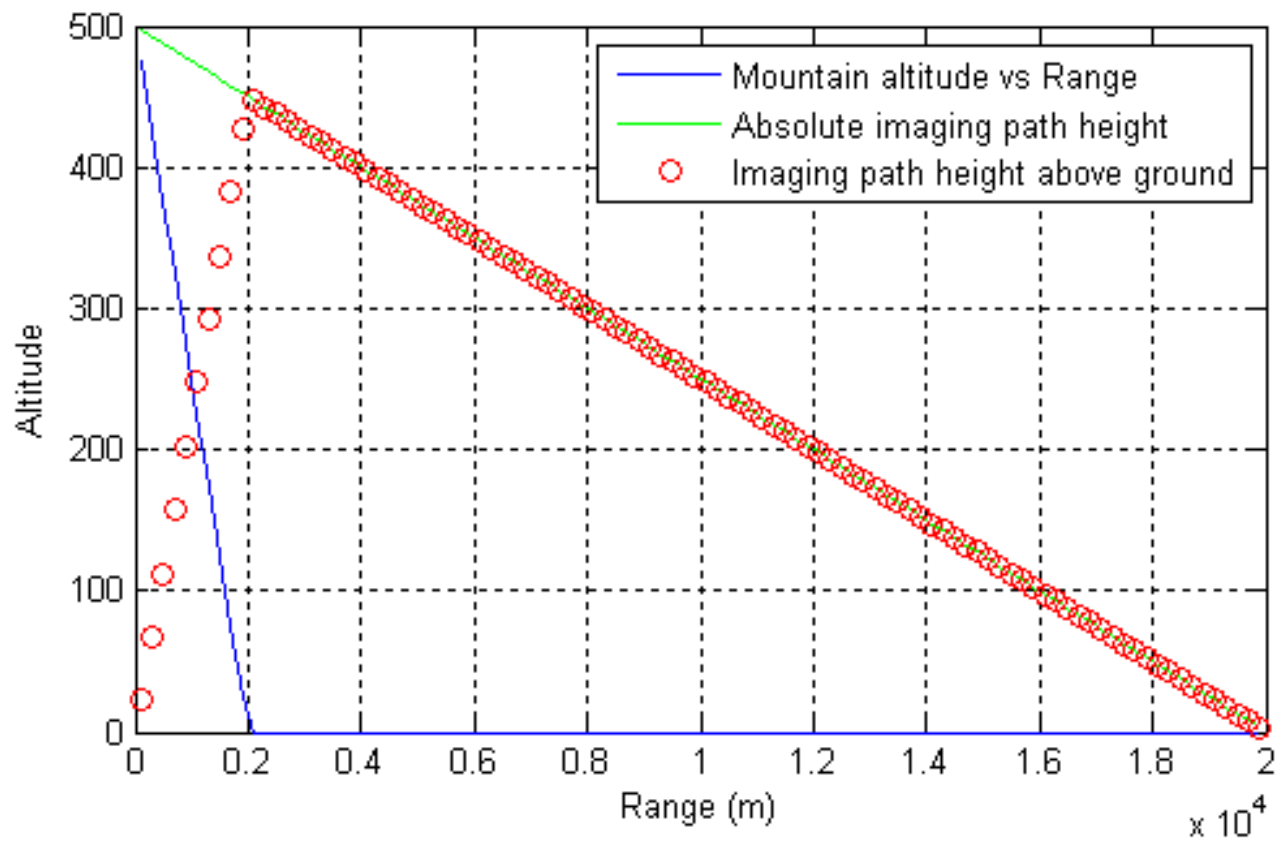

Figure 16a: Plot of example mountain model altitude profile for the $20 \mathrm{~km}$ case with a $500 \mathrm{~m}$ tall mountain with a $2 \mathrm{~km}$ base radius. 


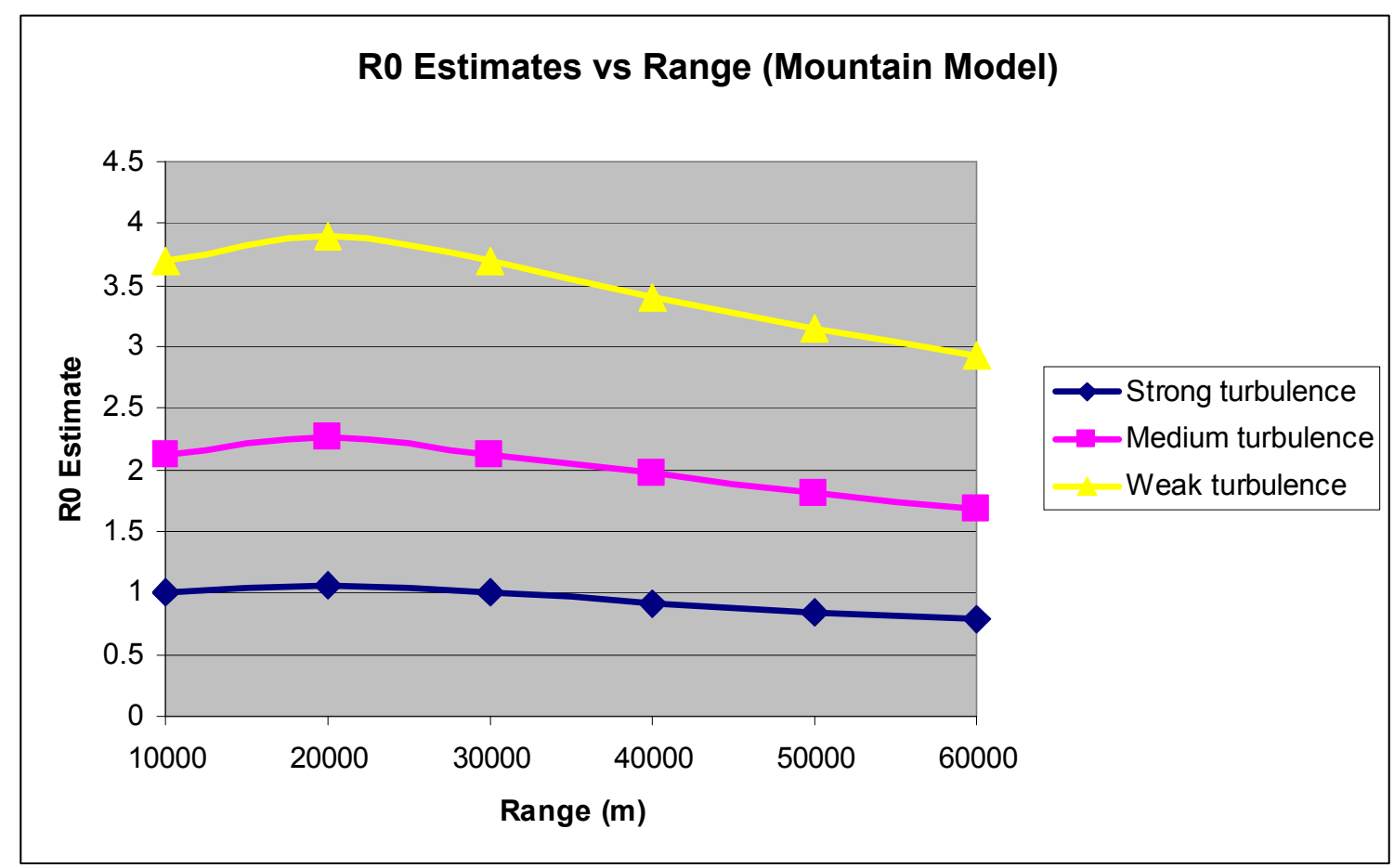

Figure 16b: $R_{0}$ estimates for typical values of $C_{n}^{2}$ over land for a slant path from 500 meters altitude looking down from a mountain with a $2 \mathrm{~km}$ radius.

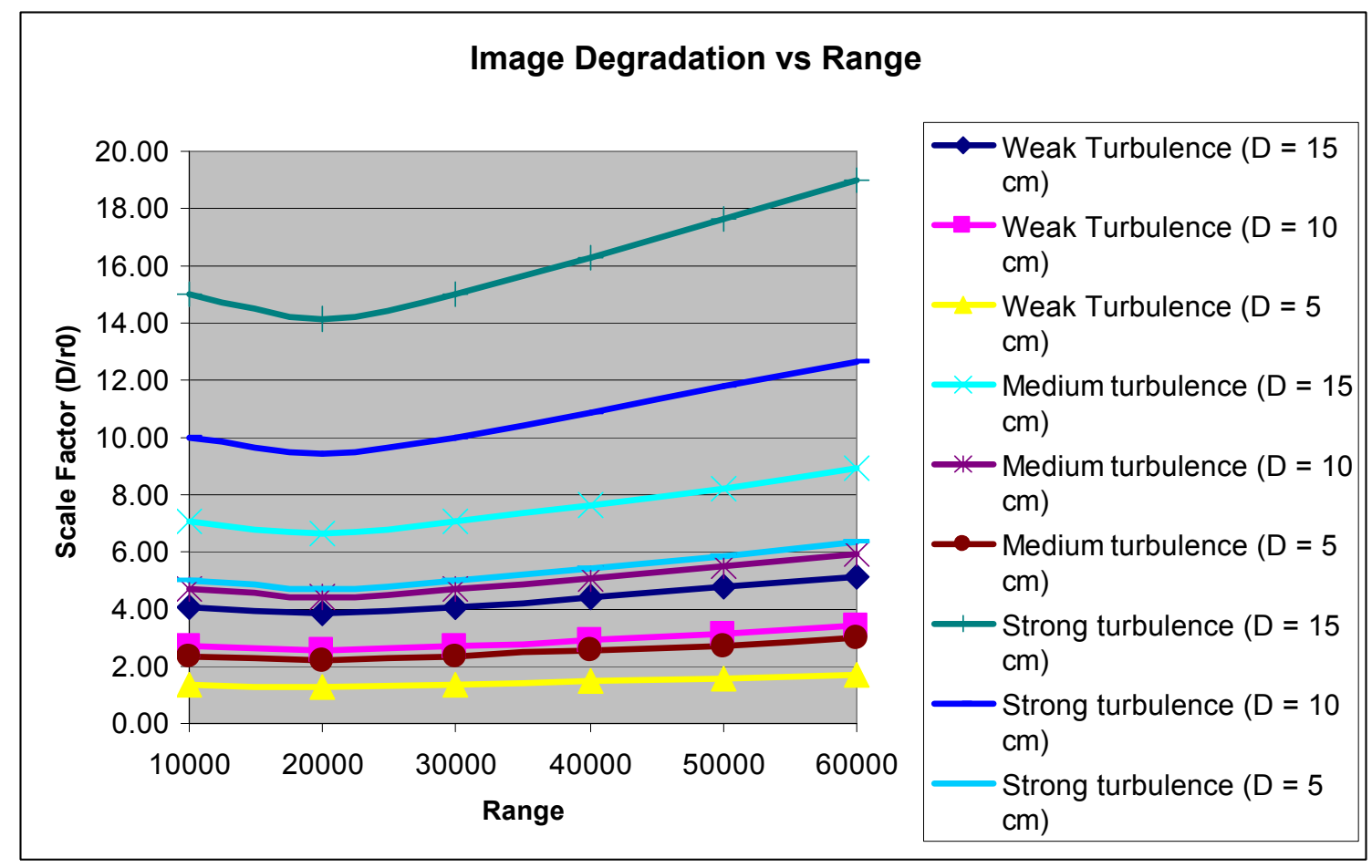

Figure 17: Image resolution degradation (or needed improvement) scale factor vs range for three aperture sizes over land using the $r_{0}$ values from Figure 16. 


\subsection{Comparison of real data in this regime with simulation}

In order to validate the simulations that will be shown, we take a look at some real data acquired in a similar regime to the charts. The data shown here was taken in from the top of Mt. Diablo in CA, whose altitude is 3849 feet (or $1050 \mathrm{~m}$ above the Livermore). We had the telescope looking on a $29 \mathrm{~km}$ slant path downwards towards Livermore on a cool fall day. Our telescope system was a $20 \mathrm{~cm}$ diameter Celestron configured without any Barlow lens, giving an effective focal length of 2 meters. Our QImaging CCD camera had a pixel size of $6.7 \mu \mathrm{m}$. This means that our pixel size on target was $\sim 9.7 \mathrm{~cm}$ and since the diffraction limit of the telescope at $29 \mathrm{~km}$ range is $7.25 \mathrm{~cm}$, we were under Nyquist-sampled by a factor of 2.6. The idealized altitude profile is shown in Figure 18a and an example short exposure frame at $50 \%$ scale from the 100 frame sequence is shown in Figure 18b. Notice how noisy the raw data is; the data was acquired on a very hazy day, thus the scene contrasts are reduced dramatically. The result of summing all 100 frames together after they are registered is shown in Figure 18c. Notice how the frame averaging reduces the noise. The highresolution speckle processed result is given in Figure 18d. The $r_{0}$ estimated from the speckle processing is roughly $1 \mathrm{~cm}$. Using the mountain slant-path geometry and a mountain radius of $10 \mathrm{~km}$, that works out to a $\mathrm{Cn} 2$ of $1.2 \mathrm{e}-12$ at the ground level. This is a reasonable estimate for $\mathrm{C}_{\mathrm{n}}{ }^{2}(0)$ and corresponds to a value in our medium turbulence case considered here. Because the pixel size we used that day was larger by $3 x$ than the earlier real data example, we needed to reduce the exposure time roughly $3 \mathrm{x}$, which for this case it was set to $1 \mathrm{~ms}$.

We next show in Figure 19 the USAF target simulated with an $r_{0}$ of $1 \mathrm{~cm}$ with similar contrast and noise levels to the real data (i.e. we used the $60 \% / 40 \%$ contrast target with 5000 peak photons per half-diffraction-limited pixel). Again the estimated isoplanatic angle is far below the diffraction limit and $\mathrm{D} / \mathrm{r} 0=20$ so we expect a reduction in the achievable resolution by a factor of 5 or so, which is shown in Figure 19d. Notice how in this case that limiting the resolution decreases the noise in the reconstruction as compared to Figure 19c, which has no bandlimiting.

The two data sets are shown at the same spatial scale. For a spatial calibration reference, at $29 \mathrm{~km}$, the large square on the USAF target imagery would be about 2.6 meters on a side. 


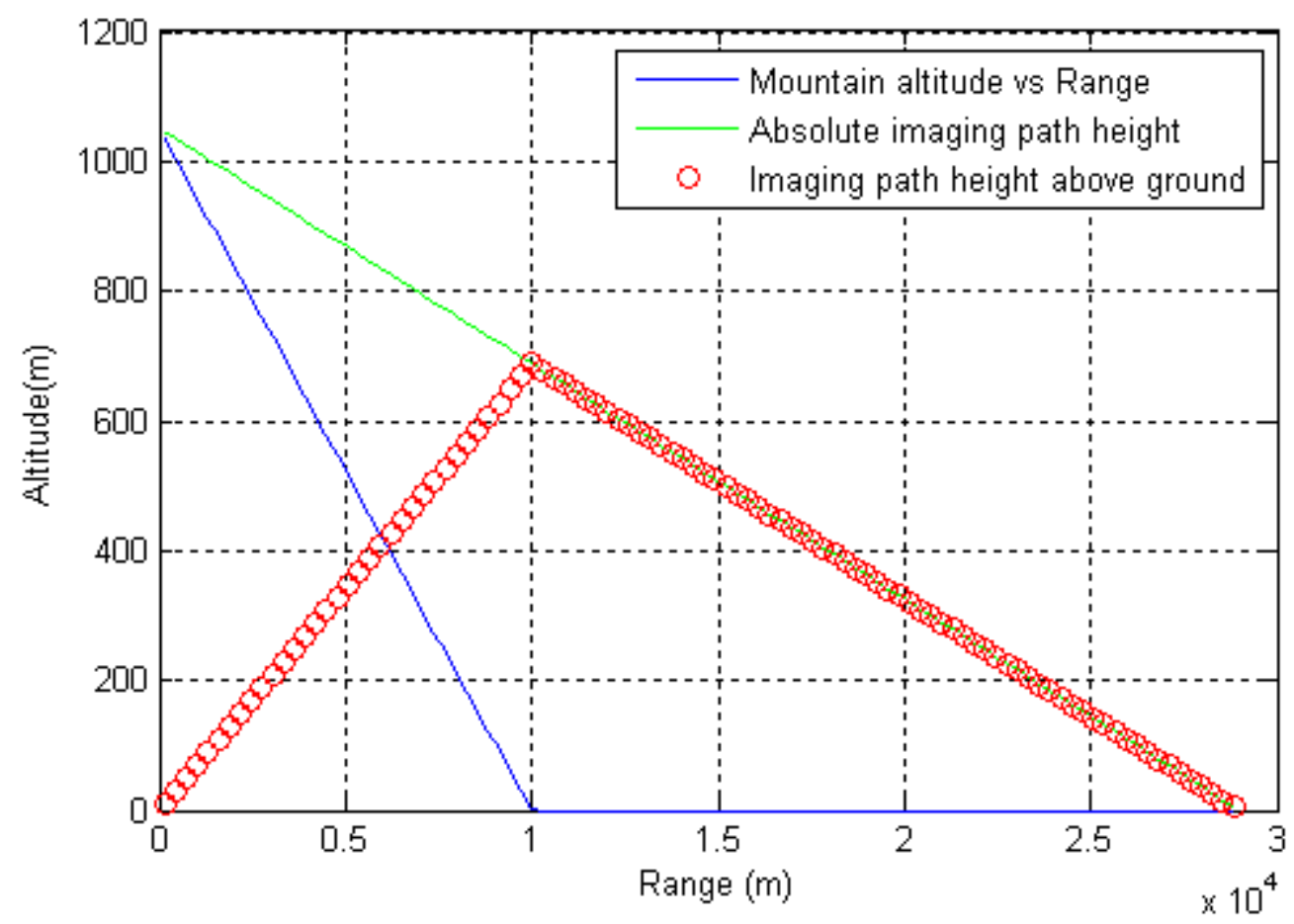

Figure 18a: Plot of Mt. Diablo altitude profile for imaging toward LLNL.

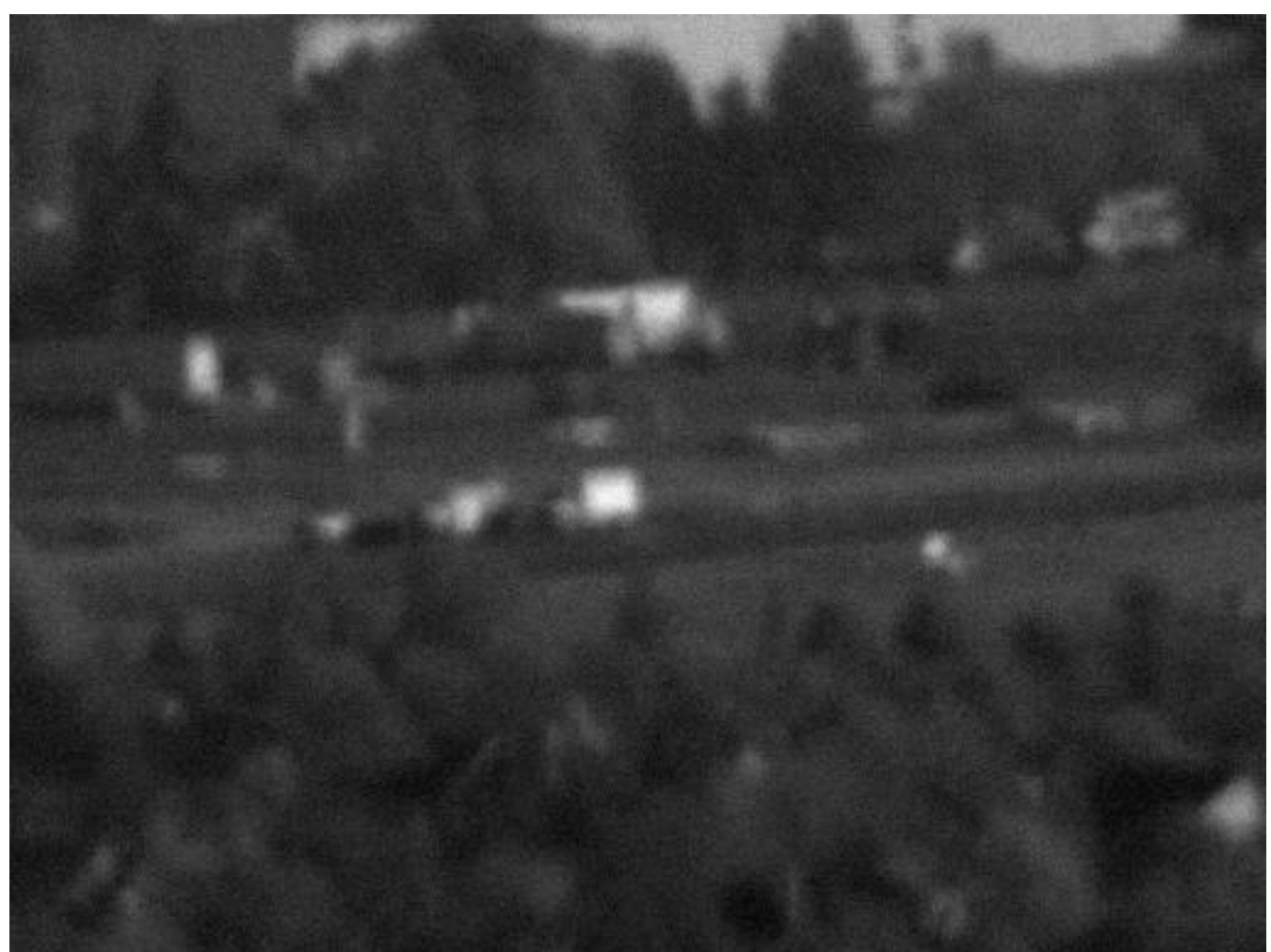

Figure 18b: Sample frame from real data collected from Mt. Diablo, CA 


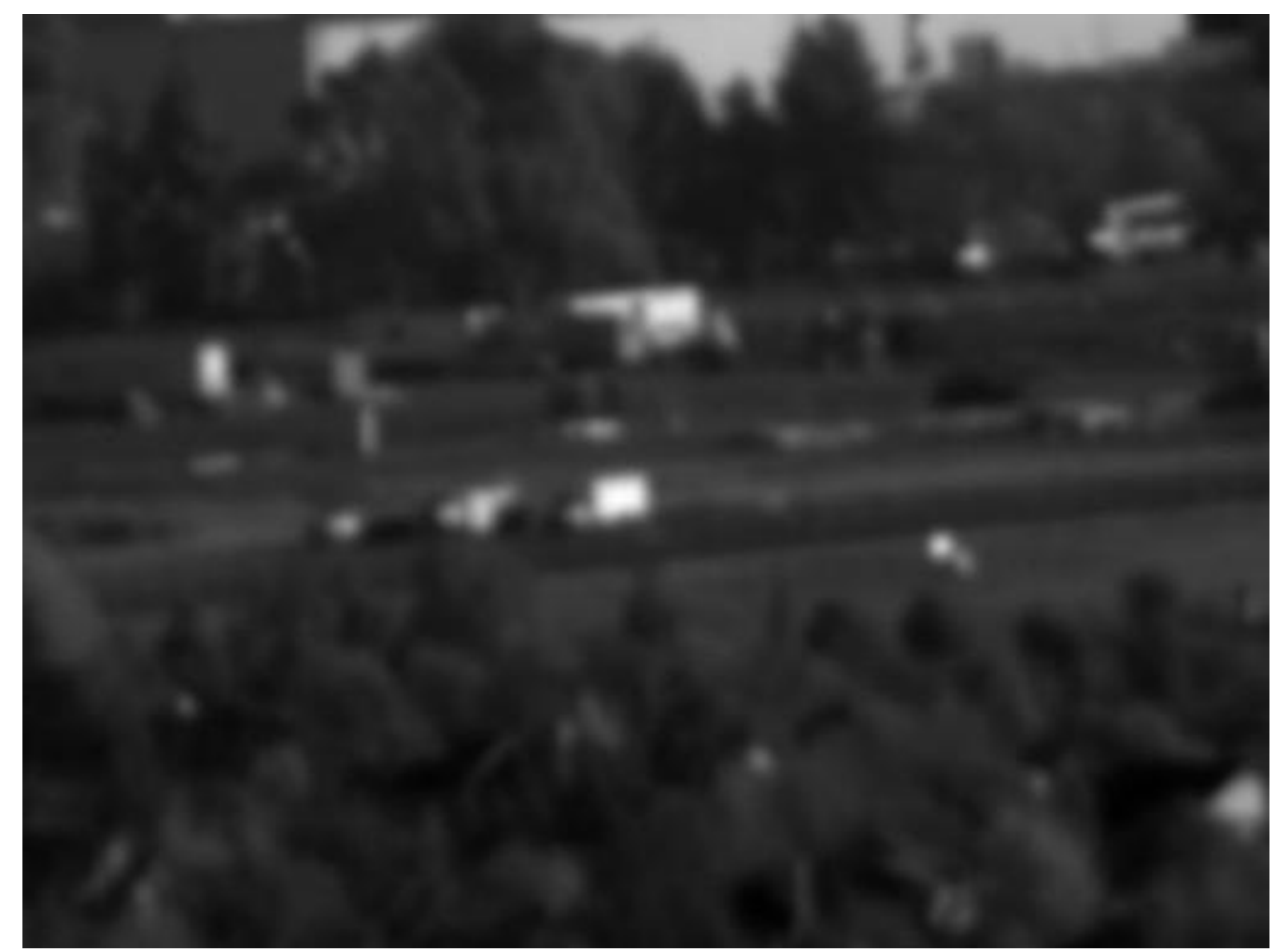

Figure 18c: Shift-and-add 100 frames

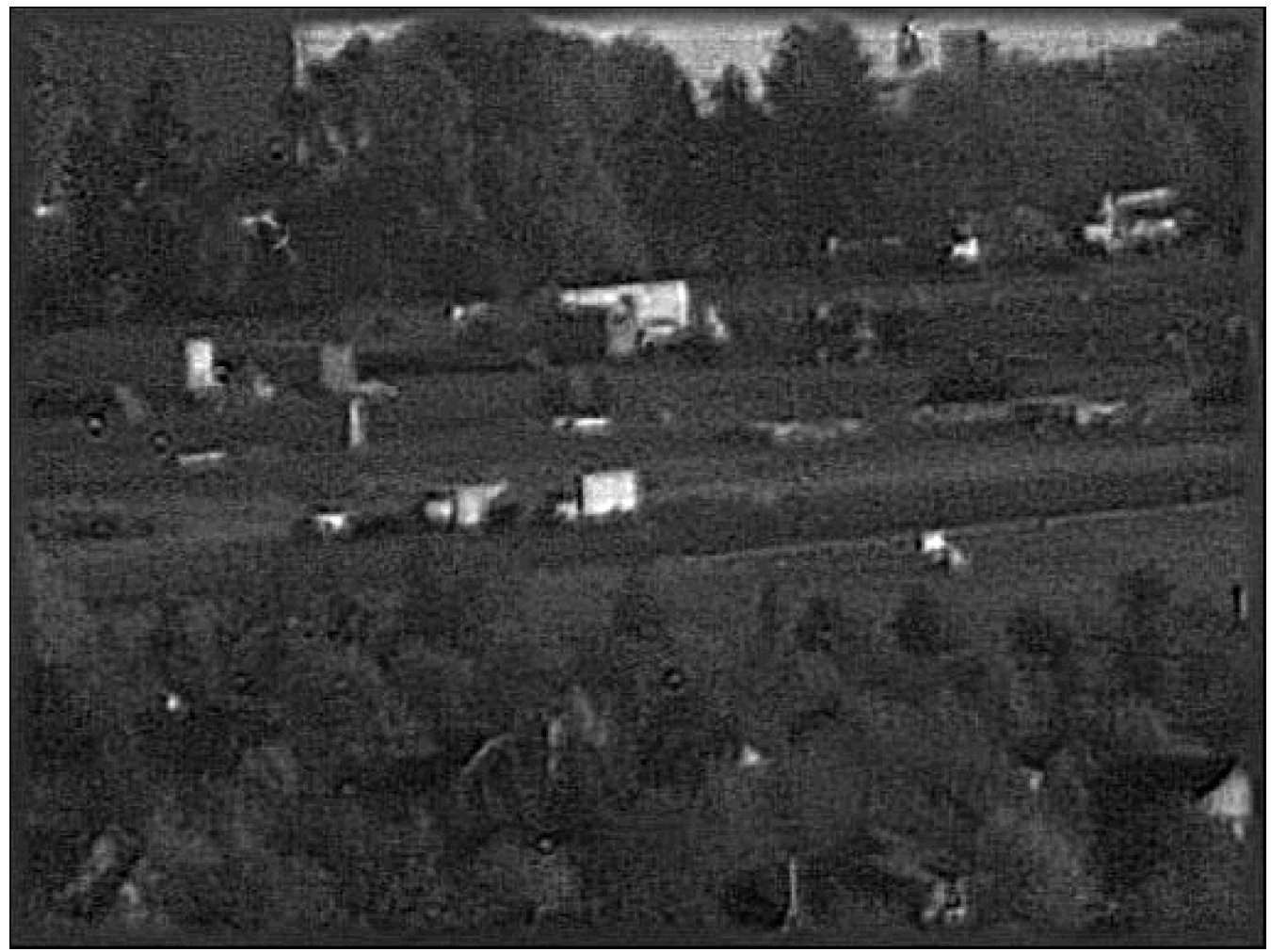

Figure 18d: Speckle processed from 100 frames 

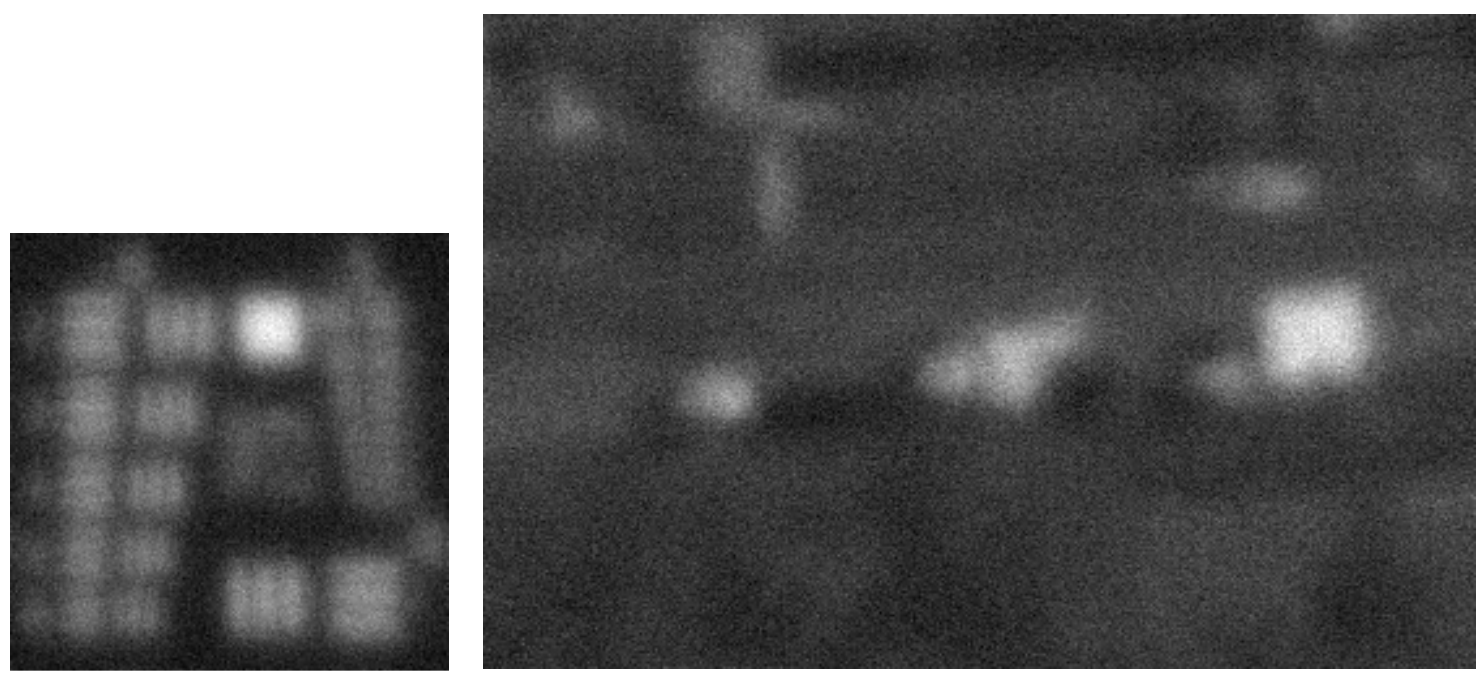

Sample simulated frame

Real data at same (full) spatial scale

Figure 19a

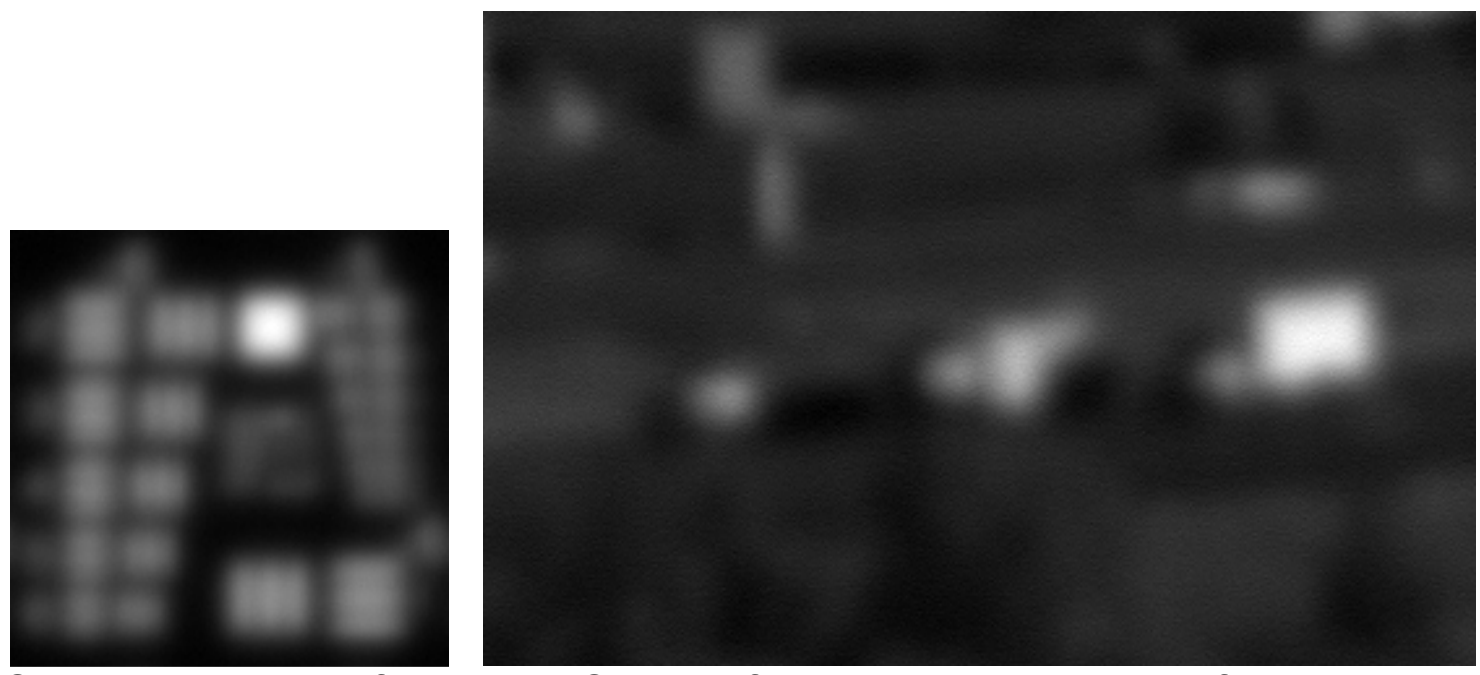

Sum 100 simulated frames

Sum (shift-and-add) 100 real data frames

Figure 19b 


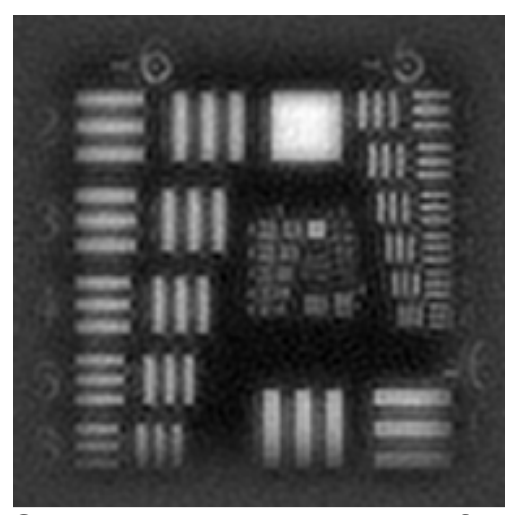

Speckle processed 100 frames

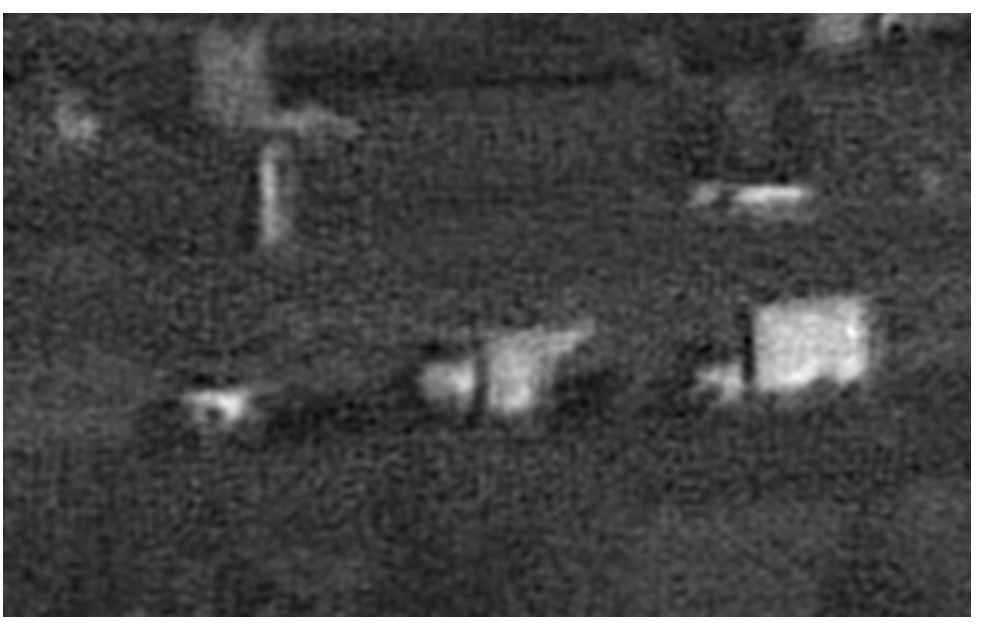

Speckle processed real data

Figure 19c: Left image was speckle processed with first order anisoplanatism effect ( $5 \mathrm{x}$ bandwidth reduction)

\subsection{Simulated imagery and effect of speckle processing}

We now do a similar set of simulations to that in section 1.5. We will look at halfdiffraction limit sampled imagery for a $15 \mathrm{~cm}$ aperture at three different contrast levels given realistic full solar illumination. Due to the way the values are parameterized, Table 2 is exactly the same as Table 1.

\begin{tabular}{|l|l|}
\hline Foreground reflectivity & Background reflectivity \\
\hline $100 \%\left(8.8 \times 10^{6} \mathrm{phot} / \mathrm{sec}\right)$ & $0 \%$ \\
\hline $60 \% \quad\left(5.2 \times 10^{6} \mathrm{phot} / \mathrm{sec}\right)$ & $40 \% \quad\left(3.5 \times 10^{6} \mathrm{phot} / \mathrm{sec}\right)$ \\
\hline $53 \% \quad\left(4.7 \times 10^{6} \mathrm{phot} / \mathrm{sec}\right)$ & $47 \% \quad\left(4.1 \times 10^{6} \mathrm{phot} / \mathrm{sec}\right)$ \\
\hline
\end{tabular}

Table 2: Target reflectivity values under consideration and associated photon flux for half-diffraction-limited sampling assuming a $60 \%$ efficient CCD, visible wavelength.

Since the $r_{0}$ values do not vary much between $10 \mathrm{~km}$ and $40 \mathrm{~km}$, for those ranges, we will simulate an $r_{0}$ of $2 \mathrm{~cm}$ for the medium turbulence case and an $r_{0}$ of $1 \mathrm{~cm}$ for the strong turbulence case. We will then look at the $60 \mathrm{~km}$ case.

\section{$10 \mathrm{~km}$ to $40 \mathrm{~km}$ cases, Medium turbulence $\left(\mathrm{r}_{0}=2.0 \mathrm{~cm}\right), \mathrm{D}=15 \mathrm{~cm}$}

Since we will be assuming $r_{0}$ to be the same for each of these cases, the only thing that really changes versus range here is the spatial scale:

- For the $20 \mathrm{~km}$ case, the large square in between and just below the -6 and -5 has dimensions of $2.4 \mathrm{~m} \times 2.4 \mathrm{~m}$. The next smaller square, in between and just below the -4 and -3 has dimensions of $60 \mathrm{~cm}$ by $60 \mathrm{~cm}$.

- For the $40 \mathrm{~km}$ case, the large square in between and just below the -6 and -5 has dimensions of $4.8 \mathrm{~m} \times 4.8 \mathrm{~m}$. The next smaller square, in between and just below the -4 and -3 has dimensions of $1.2 \mathrm{~m}$ by $1.2 \mathrm{~m}$. 
Figure 20 shows the simulated results including a sample frame, sum of aligned frames, and the ideal speckle processed result at the diffraction limit and a speckle processed result with a $3 x$ bandwidth reduction due to anisoplanatism. A $3 x$ reduction was selected because $D / r_{0}$ is 7.5 and the isoplanatic angle is well below the diffraction limit.

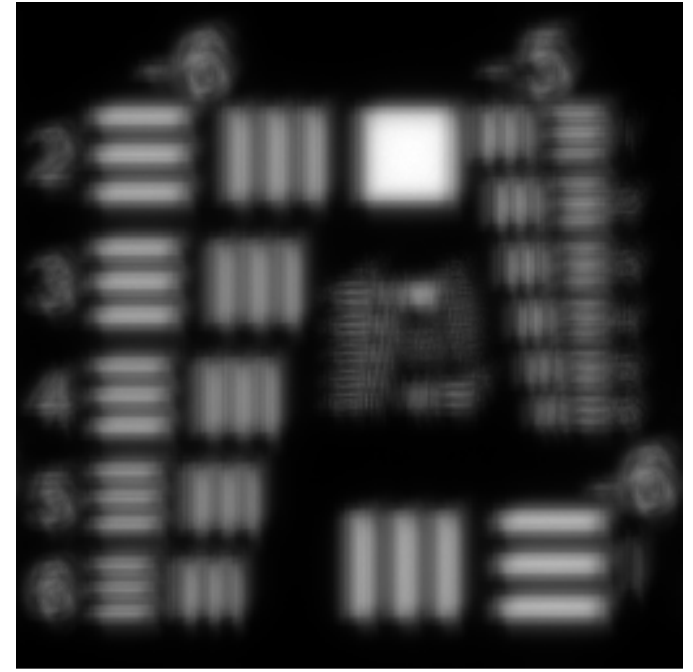

Sample frame

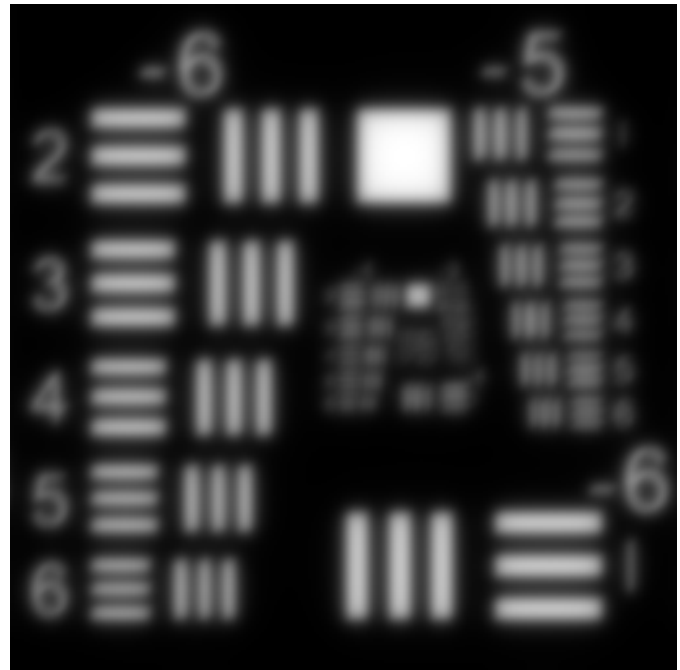

Sum of 100 aligned frames

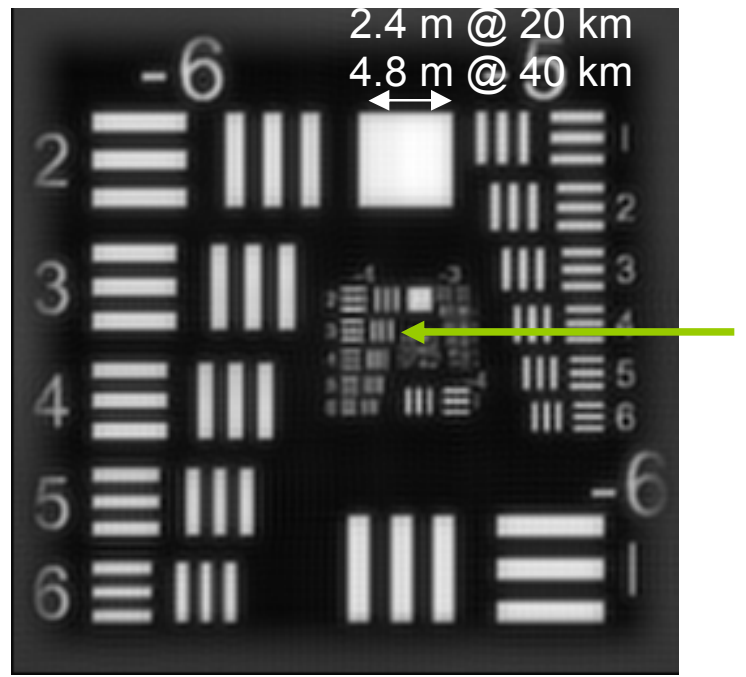

Minimum

resolvable feature size $\sim 3 \times$ D.L (20 cm@20 km or $40 \mathrm{~cm} @ 40 \mathrm{~km}$ )

Speckle processed with first-order anisoplanatism effect (3x b.w. reduction)

Figure 20a: Target contrast: $100 \% / 0 \%$, medium turbulence, $r_{0}=2 \mathrm{~cm}, D=15$ $\mathrm{cm}$, pixel size scales with range. 


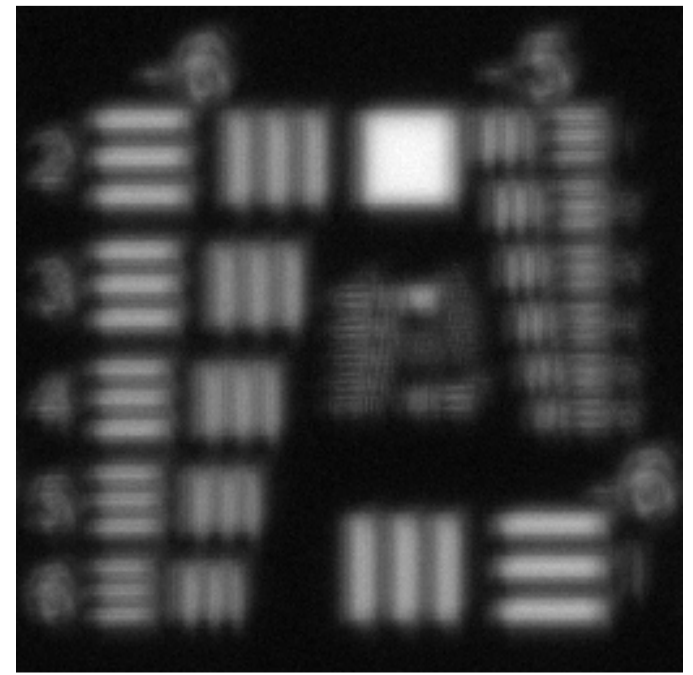

Sample frame

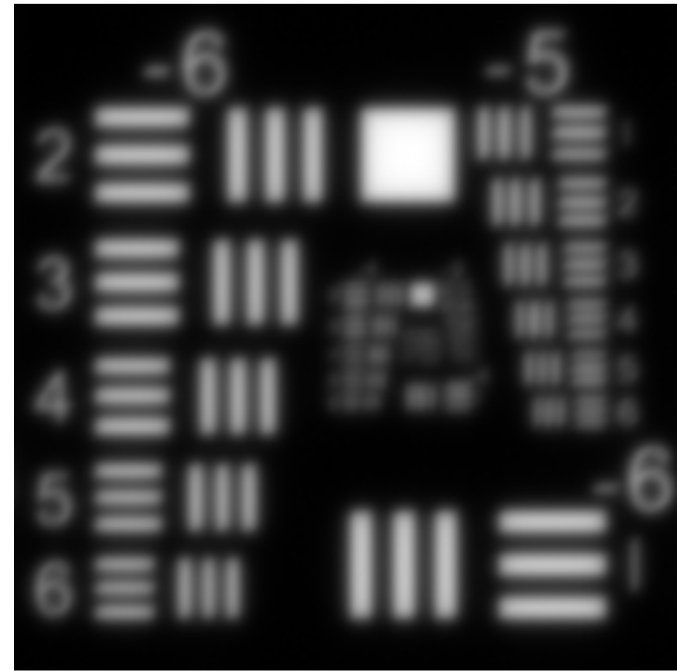

Sum of 100 aligned frames

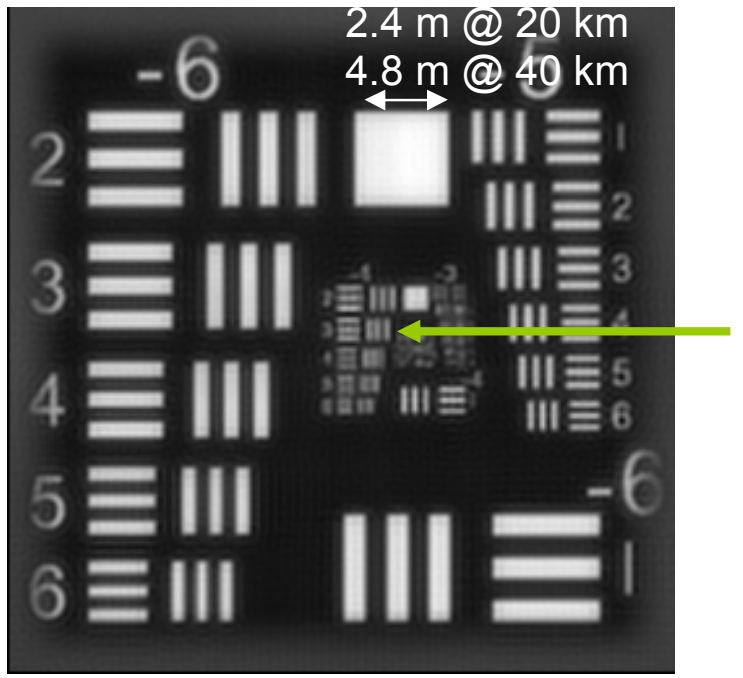

Minimum

resolvable feature

size $\sim 3 \times$ D.L

(20 cm@ 20 km or

40 cm@ 40 km)

Speckle processed with first-order anisoplanatism effect (3x b.w. reduction)

Figure 20b: Target contrast: $60 \% / 40 \%$, medium turbulence, $r_{0}=2 \mathrm{~cm}, \mathrm{D}=15$ $\mathrm{cm}$, pixel size scales with range. 


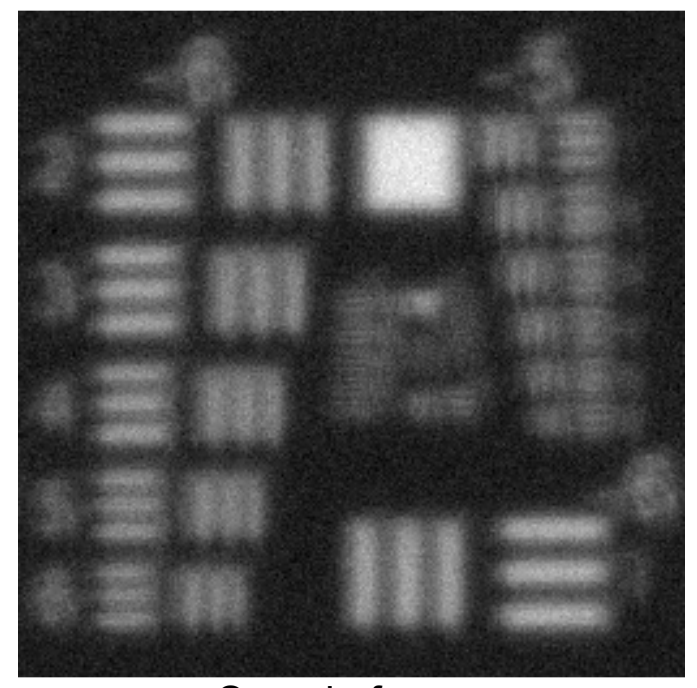

Sample frame

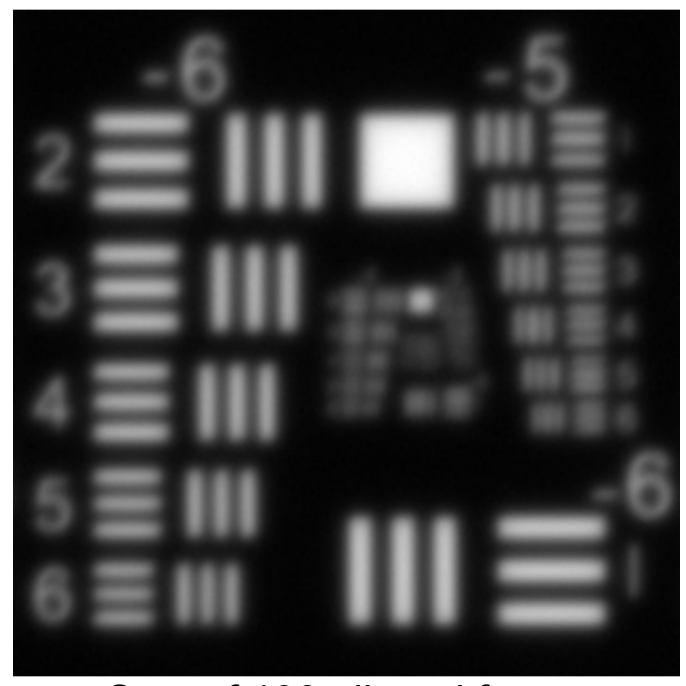

Sum of 100 aligned frames

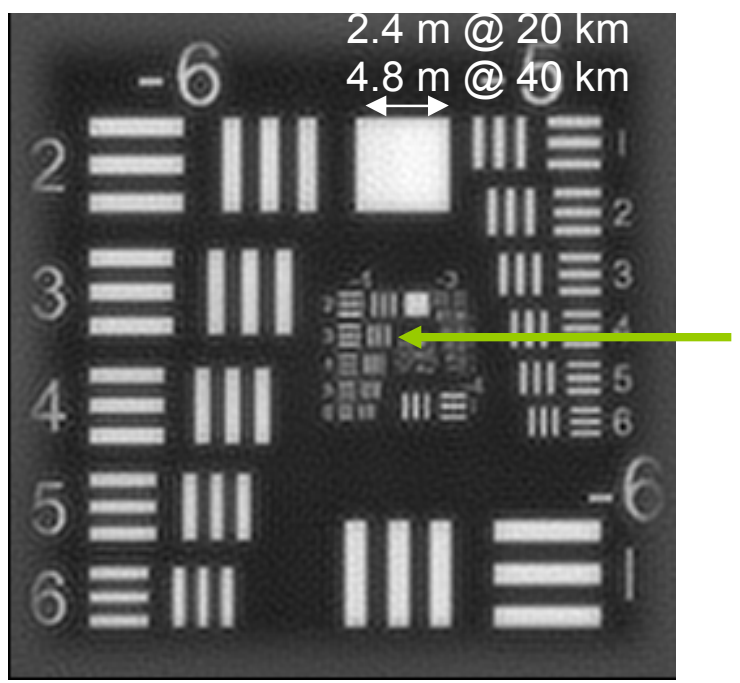

Minimum resolvable feature size $\sim 3 \times$ D.L (20 cm@ 20 km or 40 cm@ @ km)

Speckle processed with first-order anisoplanatism effect (3x b.w. reduction)

Figure 20c: Target contrast: $53 \% / 47 \%$, medium turbulence, $r_{0}=2 \mathrm{~cm}, \mathrm{D}=15$ $\mathrm{cm}$, pixel size scales with range.

\section{$60 \mathrm{~km}$ case, Medium turbulence $\left(\mathrm{r}_{0}=1.7 \mathrm{~cm}\right), \mathrm{D}=15 \mathrm{~cm}$}

For the $60 \mathrm{~km}$ case, the large square in between and just below the -6 and -5 has dimensions of $7.1 \mathrm{~m} \times 7.1 \mathrm{~m}$. The next smaller square, in between and just below the -4 and -3 has dimensions of $1.8 \mathrm{~m}$ by $1.8 \mathrm{~m}$. Again the isoplanatic angle is much smaller than the diffraction limit here and given that $D / r_{0}$ is 8.8 , we 
will apply more of a bandwidth reduction than in the previous $(10 \mathrm{~km}-40 \mathrm{~km})$ cases. Figure 21 shows only the low contrast target case.

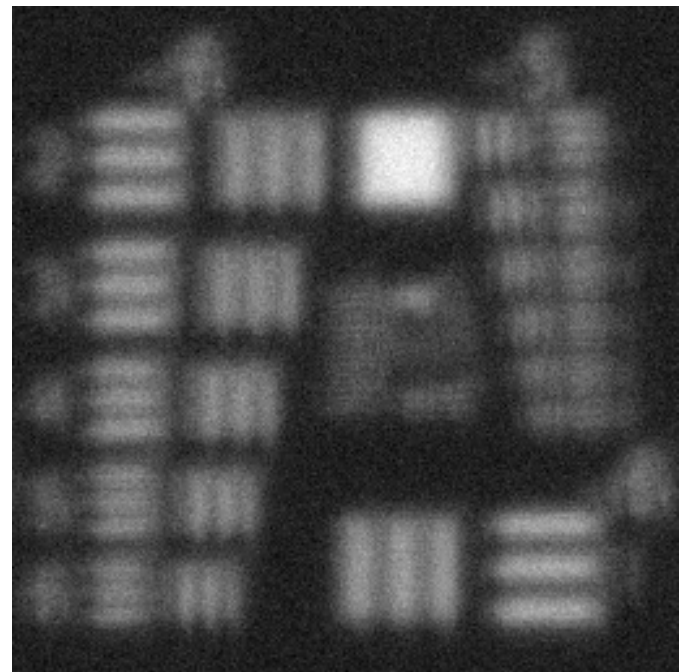

Sample frame

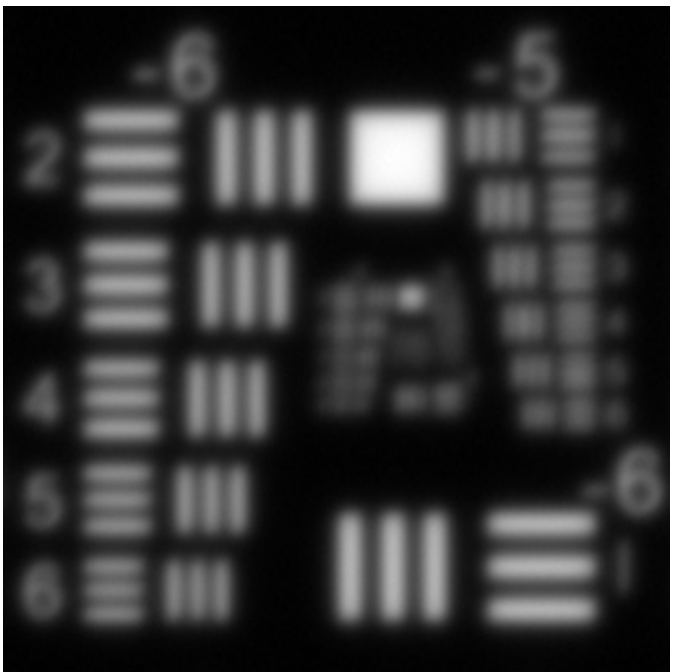

Sum of 100 aligned frames

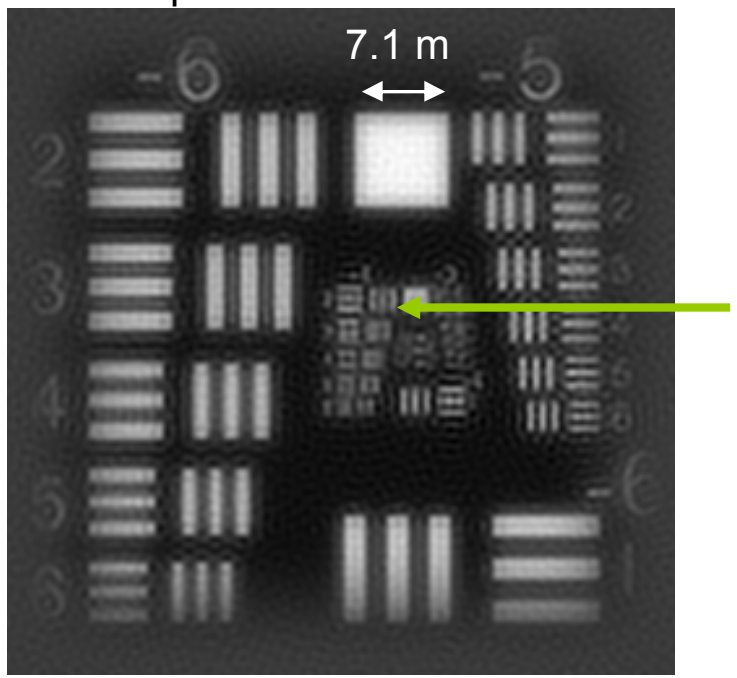

Minimum

resolvable feature size $\sim 4$ x D.L. $(80 \mathrm{~cm})$

Speckle processed with first-order anisoplanatism effect (4x b.w. reduction)

Figure 21: Target contrast: $53 \% / 47 \%$, medium turbulence, $r_{0}=1.7 \mathrm{~cm}, \mathrm{D}=15$ $\mathrm{cm}$.

\section{$60 \mathrm{~km}$ case, Strong turbulence $\left(r_{0}=0.8 \mathrm{~cm}\right), D=15 \mathrm{~cm}$}

We now show the strong turbulence case at the furthest range under consideration. Again the isoplanatic angle is much smaller than the diffraction limit here and given that $D / r_{0}$ is 18.75 , we will apply even more of a bandwidth reduction than in the previous cases ( $8 x$ reduction). Figure 22 shows the low contrast target case. 

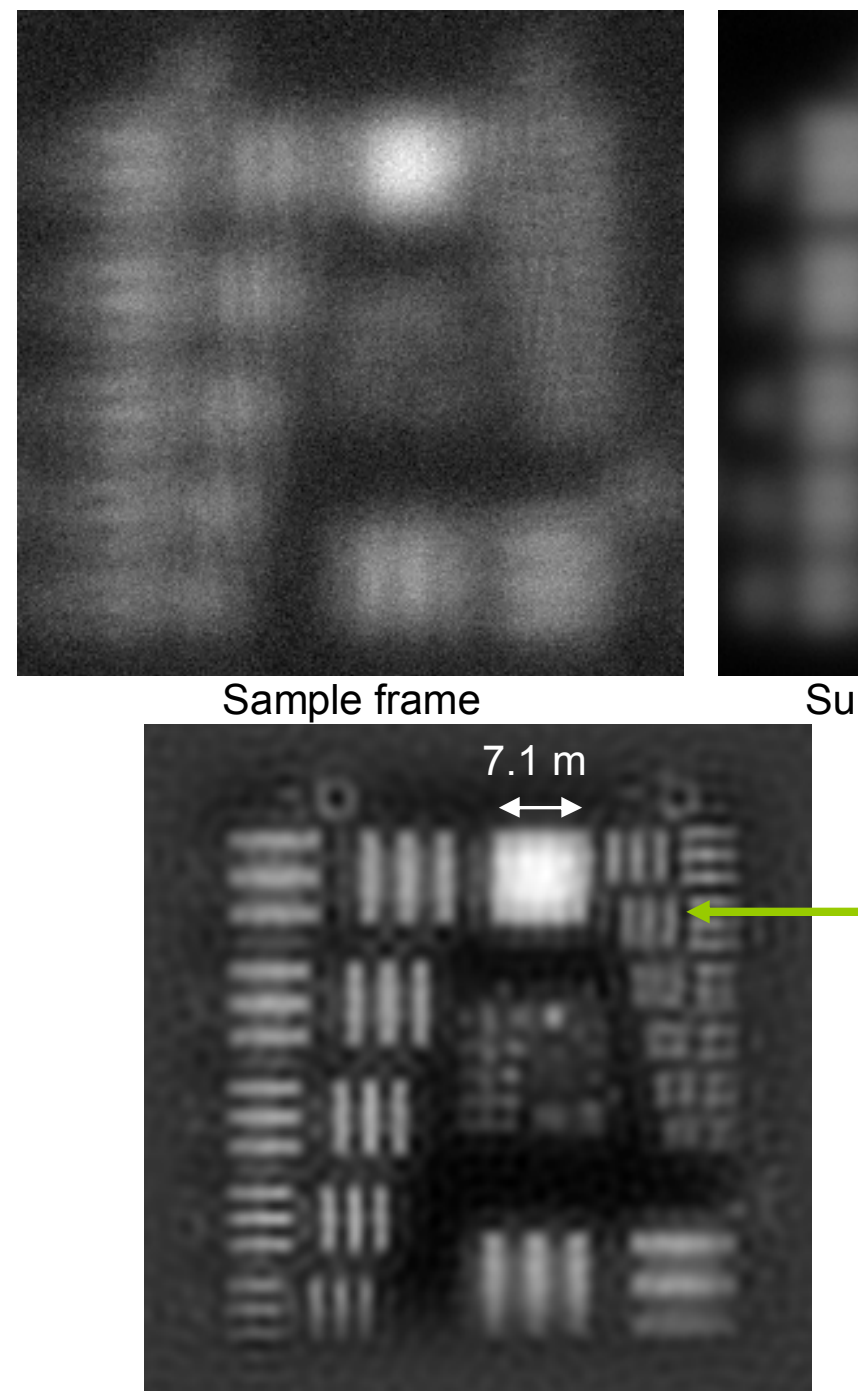

Speckle processed with first order anisoplanatism effect (8x b.w. reduction)

Figure 22: Target contrast: $53 \% / 47 \%$, strong turbulence, $r_{0}=0.8 \mathrm{~cm}, \mathrm{D}=15$ $\mathrm{cm}$.

\subsection{Wavelength dependence}

The primary advantage of to going to longer wavelengths is that atmospheric turbulence effects are decreased as wavelength gets longer. According to equation [1], $r_{0}$ scales with $\lambda^{6 / 5}$. Figure 23 and 24 give examples of how $r_{0}$ scales with wavelength for both the near and far range cases under consideration. If we then divide $\mathrm{D} / \mathrm{r}_{0}$ to give us the resolution degradation scale factor, which is also the maximum amount of improvement to be gained with image processing, we can see from Figures 25 and 26 how wavelength affects this value. If this were the only consideration, then the obvious choice would be to image at longer 
wavelengths. But, the primary disadvantage to longer wavelengths is the decreased resolution, which scales linearly with wavelength ( $d x=\lambda / D$ * range). See Figure 27 and Figure 28 for a plot of diffraction-limited resolution versus wavelength for short and longer range sets. This means that in order to achieve the same diffraction-limited resolution as wavelength increases, it is necessary to increase the aperture size, which may or may not be desirable or even possible given certain size constraints for a particular system.

Other wavelength effects are more radiometric in nature and very target dependent. Certain targets may have stronger (or weaker) reflectance, hence better or worse target to background contrast at different wavelengths.

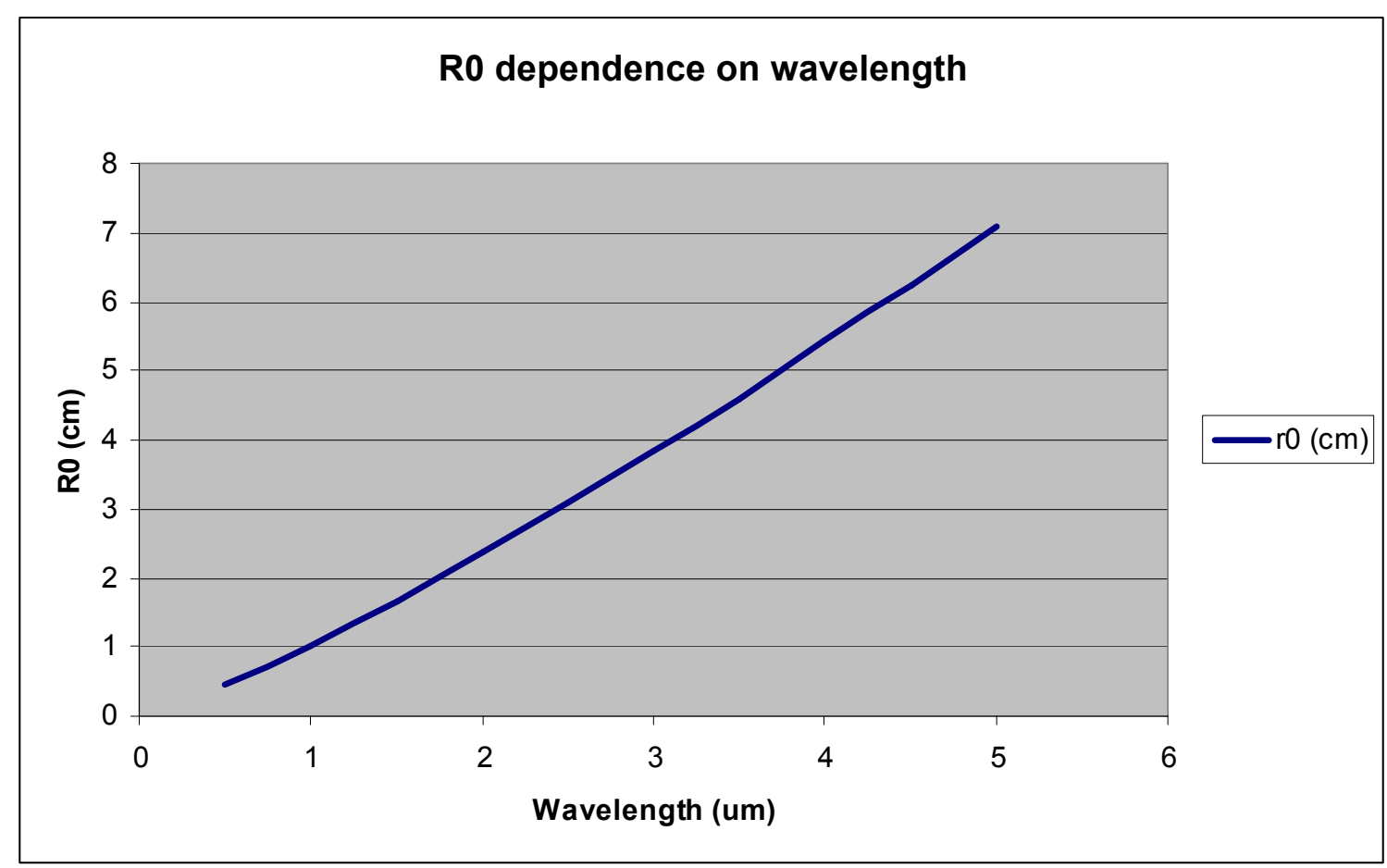

Figure 23: Plot of $r_{0}$ versus wavelength for the $C n 2=1 \times 10^{-12}$, Range $=6 \mathrm{~km}$, and a slant path from an altitude of $15 \mathrm{~m}$ 


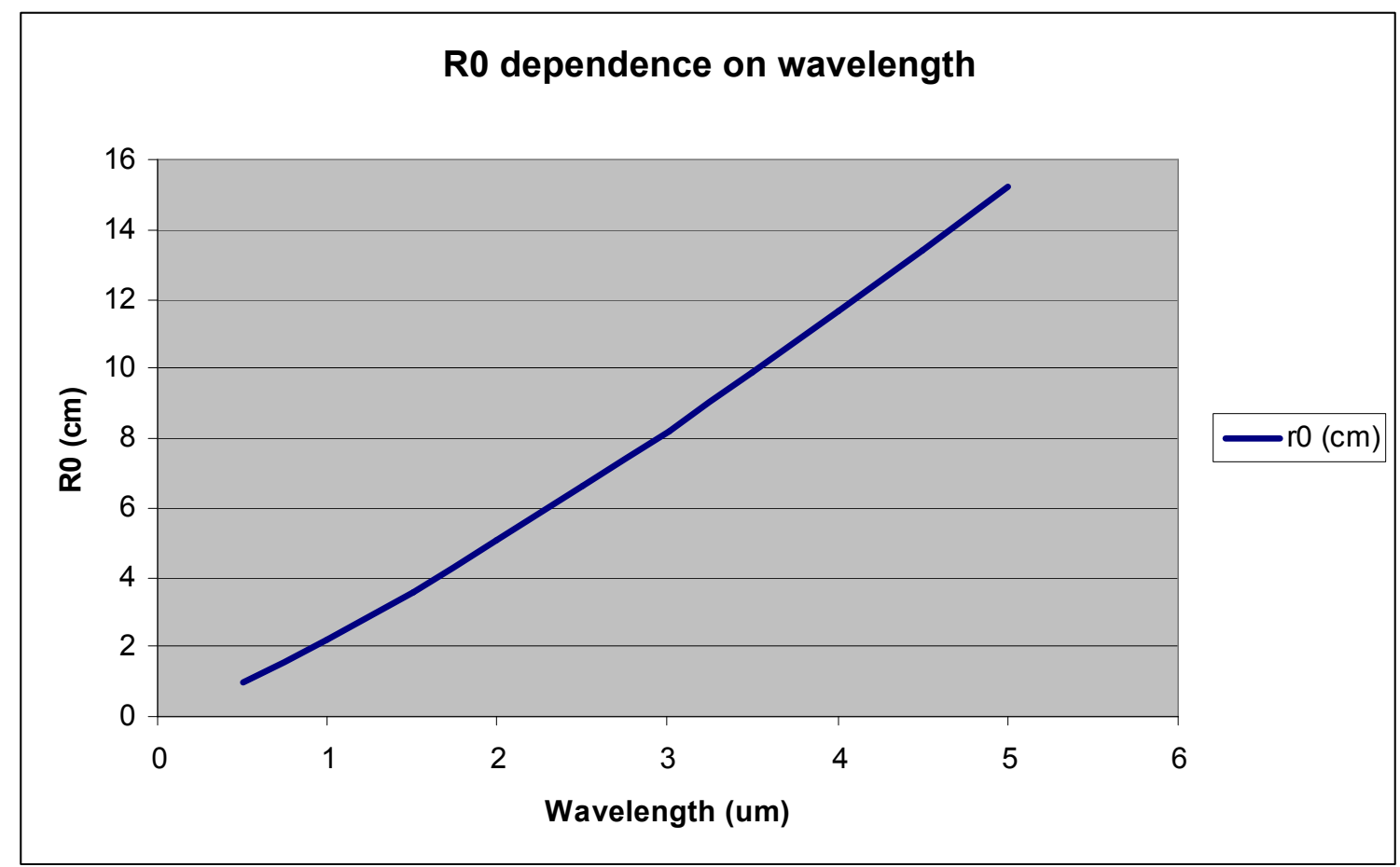

Figure 24: If $r_{0}$ in the visible $(0.5 \mu \mathrm{m})$ is $1 \mathrm{~cm}$, this plot shows how it scales up with wavelength.

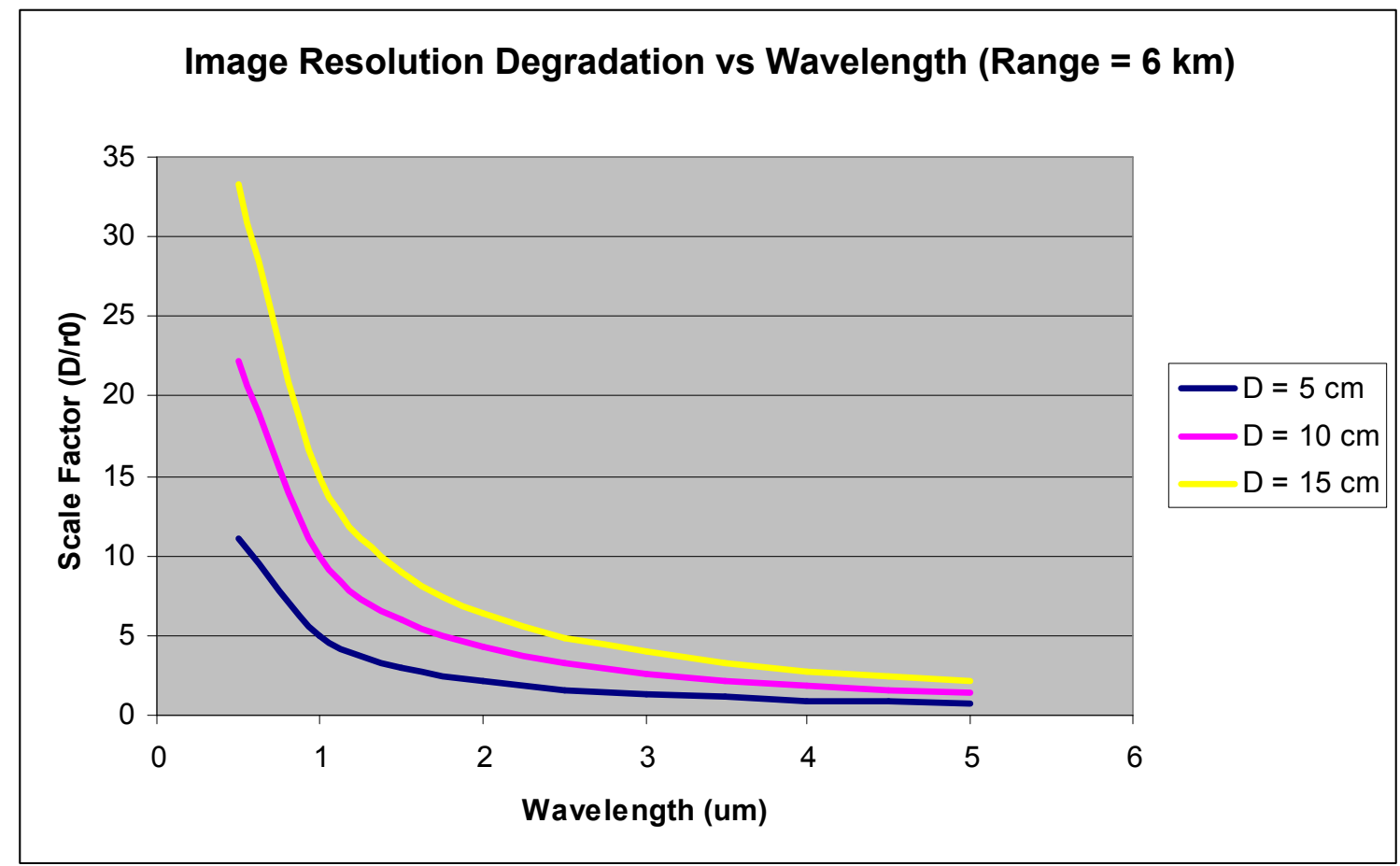

Figure 25: Plot of image resolution degradation (or needed improvement) scale factor vs. wavelength for three aperture sizes using the $r_{0}$ values from Figure 23. 


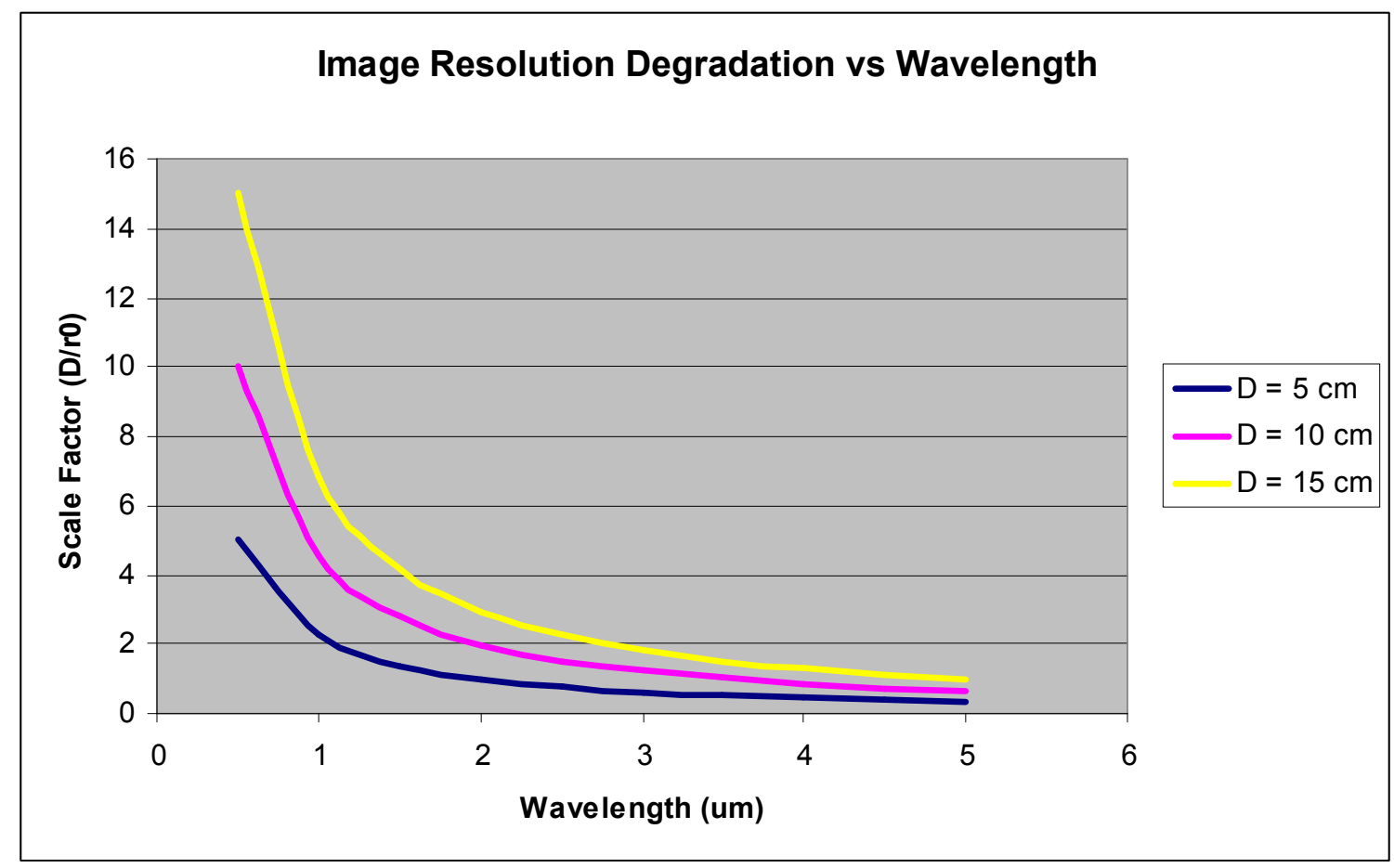

Figure 26: If $r_{0}$ in the visible $(0.5 \mu \mathrm{m})$ is $1 \mathrm{~cm}$ as in Figure 24, this plot shows how the image resolution degradation scales with wavelength for three different aperture sizes.

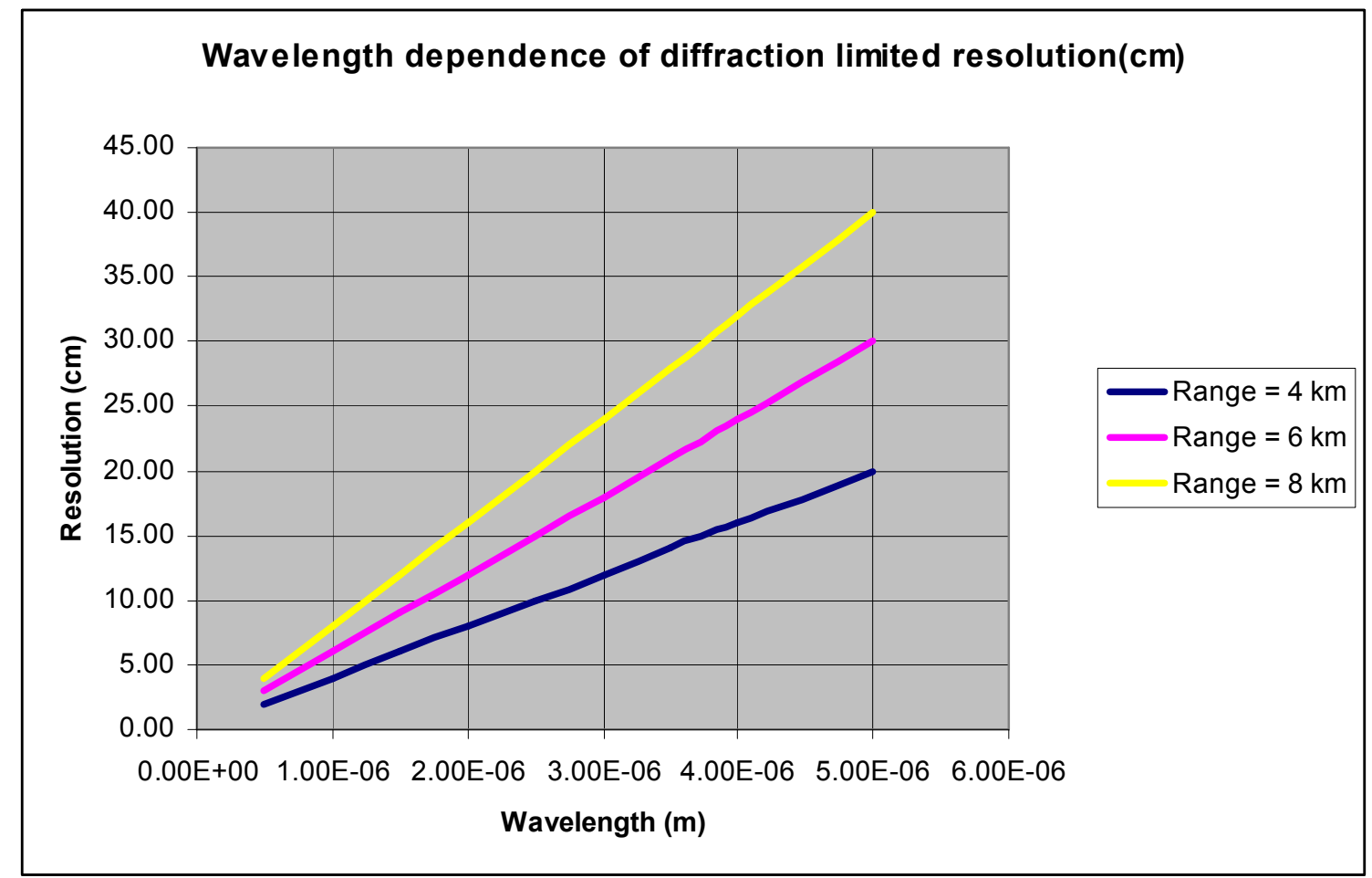

Figure 27: Plot of diffraction-limited resolution vs. wavelength for three ranges for a $10 \mathrm{~cm}$ aperture. 


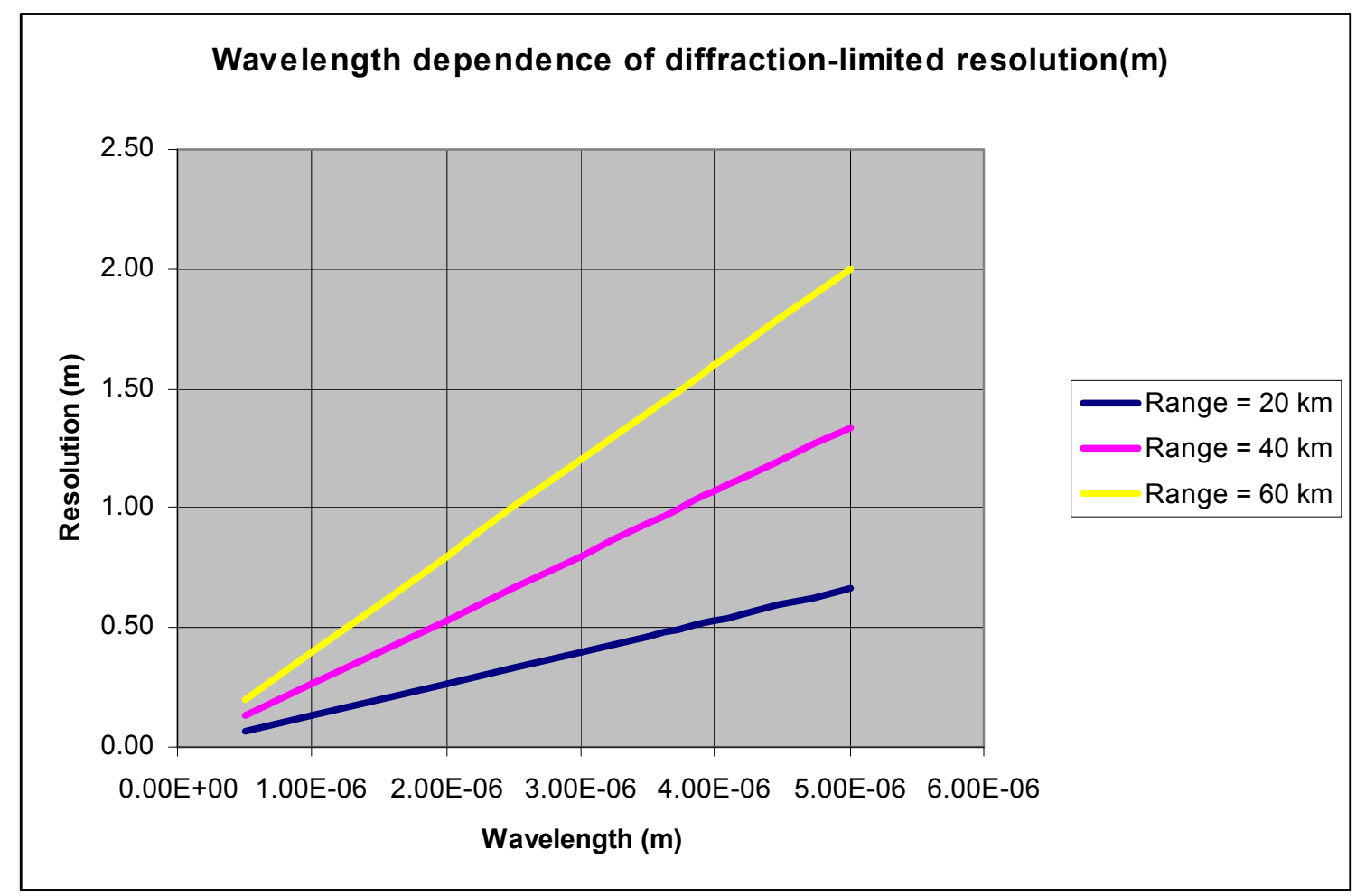

Figure 28: Plot of diffraction-limited resolution vs. wavelength for three ranges for a $15 \mathrm{~cm}$ aperture.

We now demonstrate the wavelength effects with simulated data for a selected case. If we consider the $6 \mathrm{~km}$ medium-strong turbulence case with $\mathrm{r}_{0}=$ $1 \mathrm{~cm}$ at $0.5 \mu \mathrm{m}$, we can simulate and see how the target looks at $0.5 \mu \mathrm{m}, 1.5 \mu \mathrm{m}$ and $4.0 \mu \mathrm{m}$ with a constant aperture size $(\mathrm{D}=10 \mathrm{~cm})$ before and after speckle processing (radiometric effects aside). Figure 29a shows the visible light case and Figure 29b shows the short-wave IR case. Given the turbulence levels used, both cases result in processed imagery with the same resolution since we have degraded the visible case by expected anisoplanatism. But realize that if the turbulence levels are weaker you could do better in the visible because there is still a factor of 3 in resolution to be gained to get to the diffraction limit there. The mid-wave IR case is shown in Figure 29c. No processing is needed for that case because the turbulence levels for this case are so weak in the mid-wave IR, though it is quite conceivable that the turbulence could be stronger at times or in different imaging geometries. The drawback with the mid-wave-IR is that the diffraction limit is $24 \mathrm{~cm}$ which is much larger (worse) than for either of the other two cases for the same aperture size. In order to get to the visible diffractionlimited resolutions, the aperture sizes must be scaled up which means that the $\mathrm{D} / \mathrm{r}_{0}$ will be just as bad as in the visible case shown and image processing will be needed to compensate. 

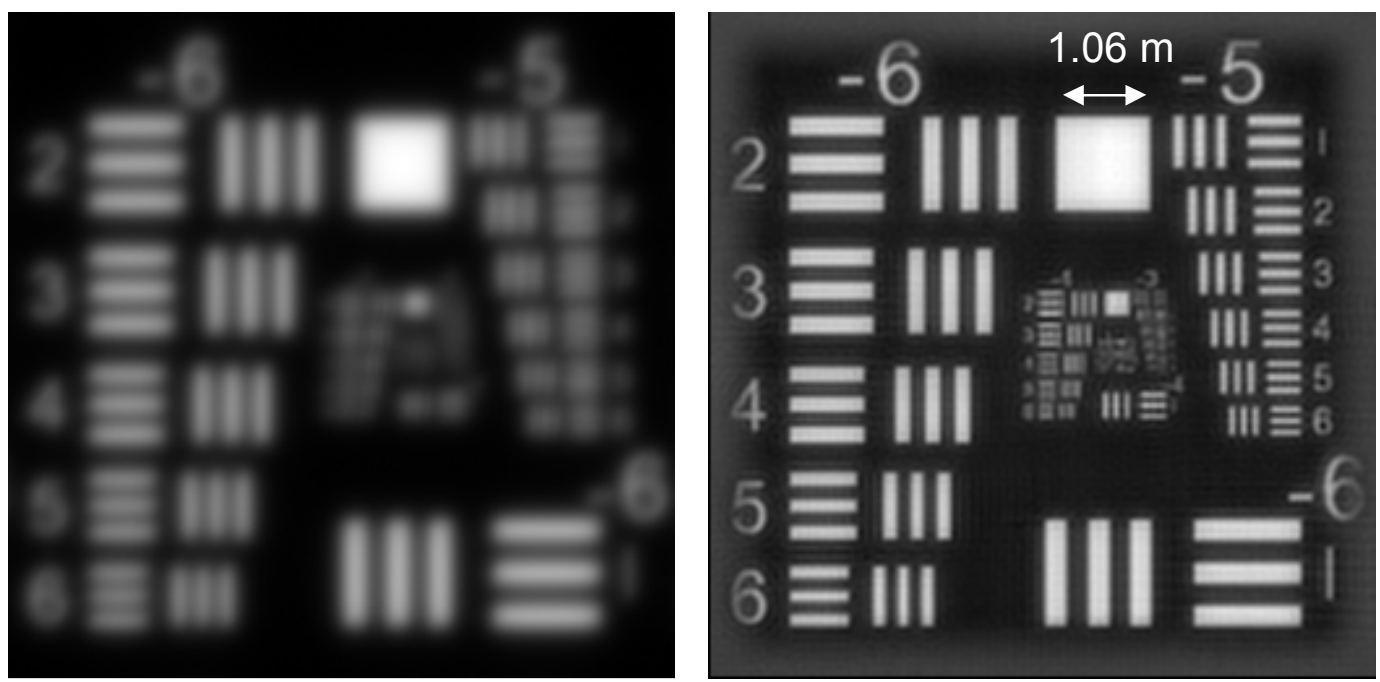

Figure 29a: Shift-and-add (left) Speckle processed (right) for $\lambda=0.5 \mu \mathrm{m}$, range $=$ $6 \mathrm{~km}$, medium-strong turbulence, $\mathrm{r}_{0}=1 \mathrm{~cm}, \mathrm{D}=10 \mathrm{~cm}, 3 \mathrm{x}$ bandwidth reduction used due to anisoplanatism, pixel size scale is shown at the diffraction limit. Minimum resolvable feature size is $3^{*}$ D.L. or $9 \mathrm{~cm}$.
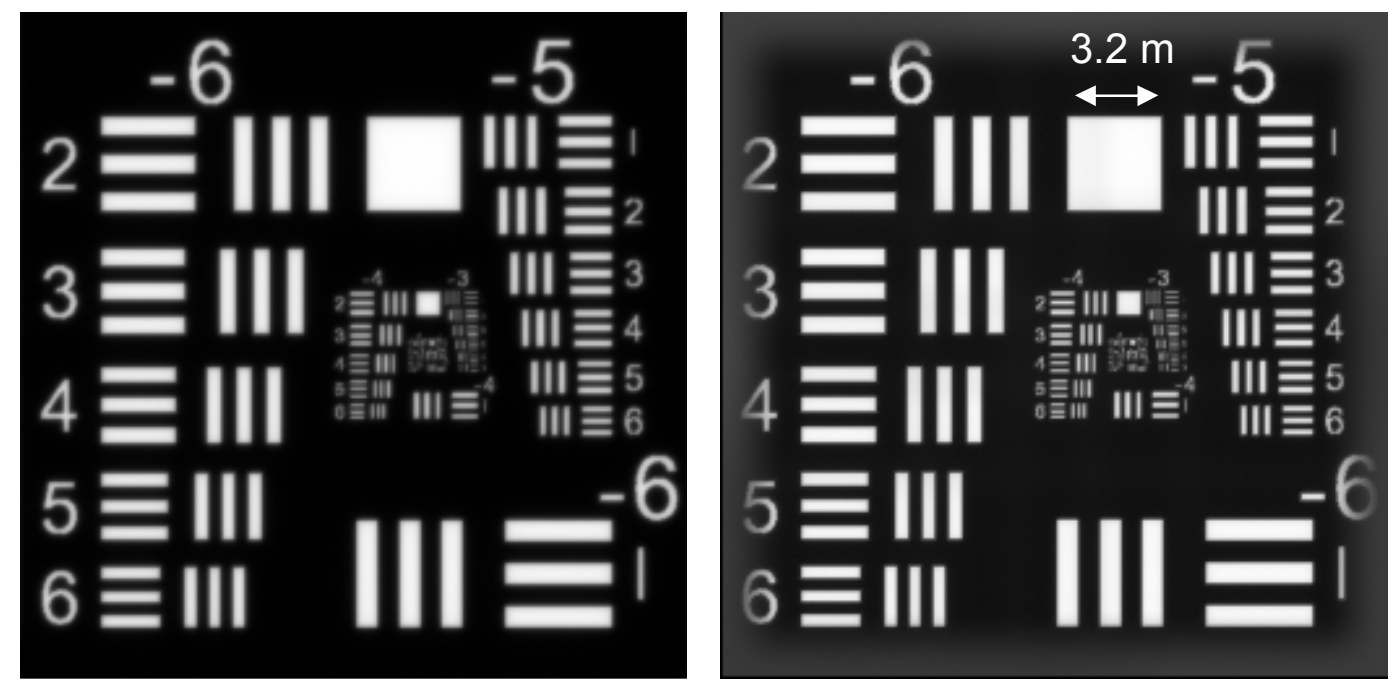

Figure 29b: Shift-and-add (left) Speckle processed (right) for $\lambda=1.5 \mu \mathrm{m}$, range $=$ $6 \mathrm{~km}$, medium-strong turbulence (in the visible), $r_{0}$ is now $3.8 \mathrm{~cm}, \mathrm{D}=10 \mathrm{~cm}$, no bandwidth reduction since $\mathrm{D} / \mathrm{r}_{0}$ is so low (2.8), pixel size scale is shown at the diffraction limit. Minimum resolvable feature size is at the D.L. or $9 \mathrm{~cm}$. 


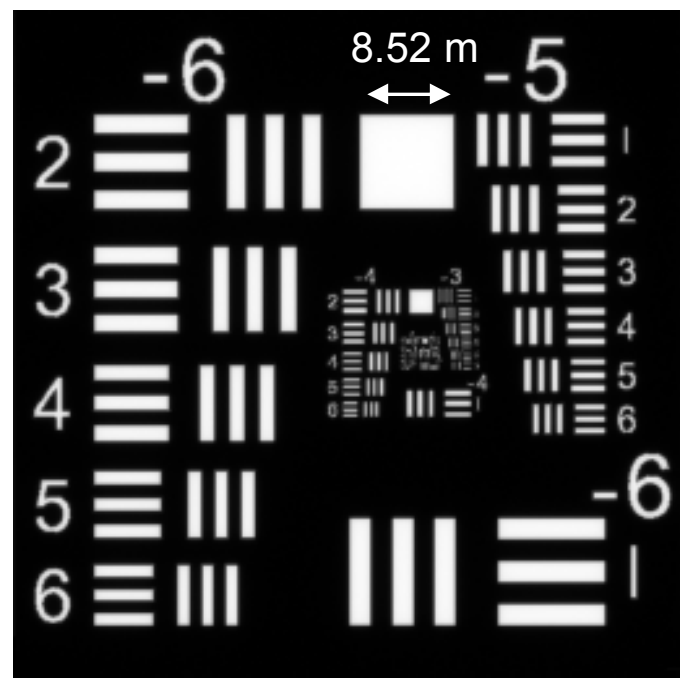

Figure 29c: Shift-and-add for $\lambda=4.0 \mu \mathrm{m}$, range $=6 \mathrm{~km}$, medium-strong turbulence (in the visible), $r_{0}$ is now $12.4 \mathrm{~cm}, D=10 \mathrm{~cm}$. Because $D / r_{0}<1 \mathrm{no}$ processing is needed. Pixel size scale is shown at the diffraction limit. Minimum resolvable feature size is at the D.L. or $24 \mathrm{~cm}$.

\subsection{Lucky imaging considerations}

Lucky imaging techniques require a large number short exposure images and utilize an image quality metric to select only images of good quality. It has been found [3] that the probability of obtaining a lucky short exposure image is Prob $5.6 \exp \left[-0.1557\left(D / r_{0}\right)^{2}\right]$ (for $D / r_{0}>=3.5$ ) See Figure 30 for a plot. What this means is that the stronger the atmosphere, the longer you will have to wait to get a high quality frame. In weak turbulence you will not have to wait nearly as long for a good frame. Now, in the anisoplanatic imaging case where the point spread function is position dependent, this approach can be refined to select high quality regions in the image, because the chances that an entire frame will be high-quality under such conditions will be nearly zero.

To give an idea of the average wait time between "good" frames or "good" regions for our scenarios, let us consider a camera operating at video frame rates, or 30 frames per second. Figure 31 plots the average wait time versus D/ $r_{0}$. If dwell time is not an issue and targets are static for a long time (i.e. seconds + for weak turbulence and minutes to hours for stronger turbulence), then lucky imaging techniques can be of help. In fact, lucky imaging can be integrated into other image processing methods such as speckle imaging as a pre-processing step to pre-select frames or regions of higher quality for it to process. 


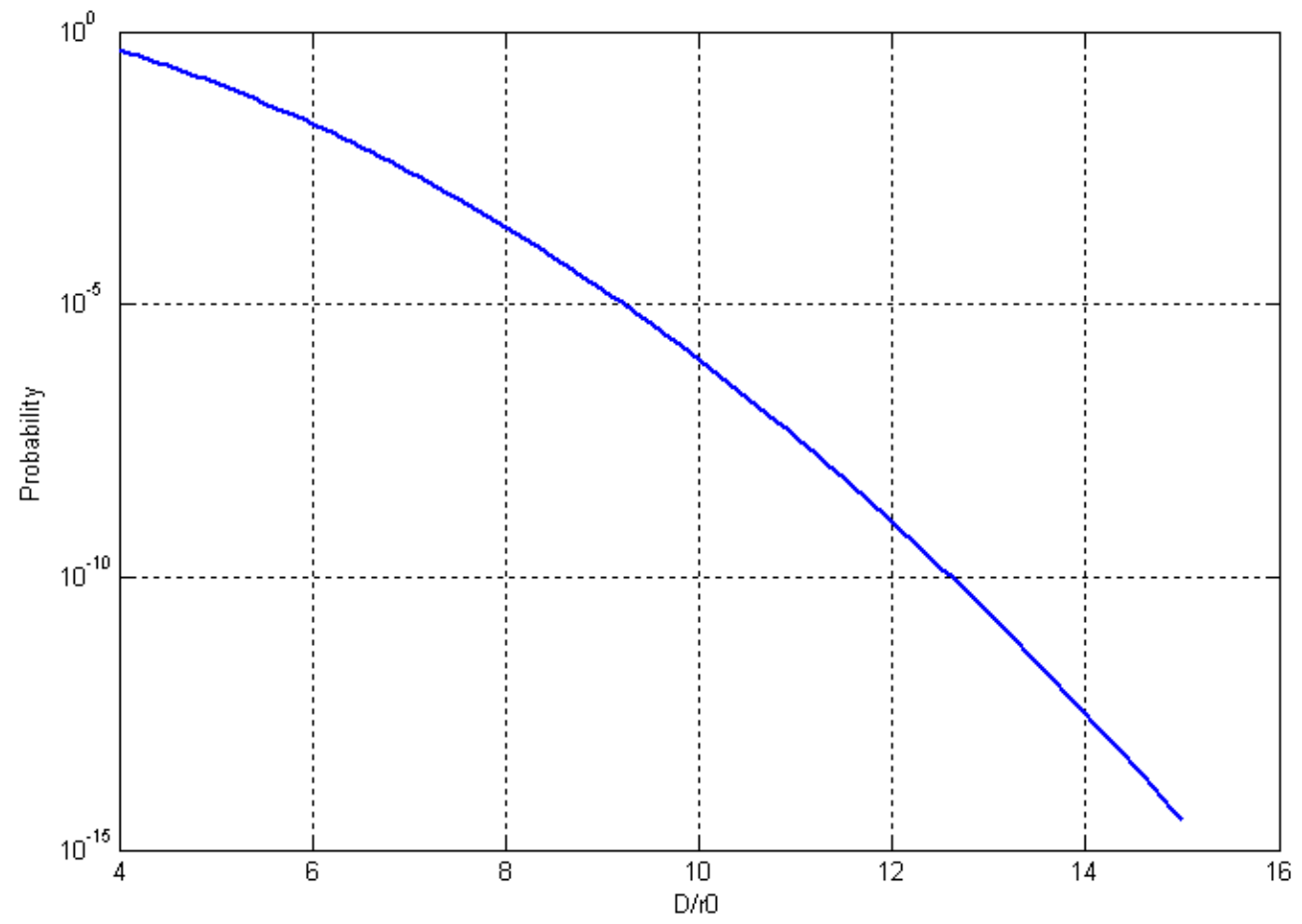

Figure 30: The probability of obtaining a lucky image as a function of the ratio of aperture diameter to $r_{0}$. 


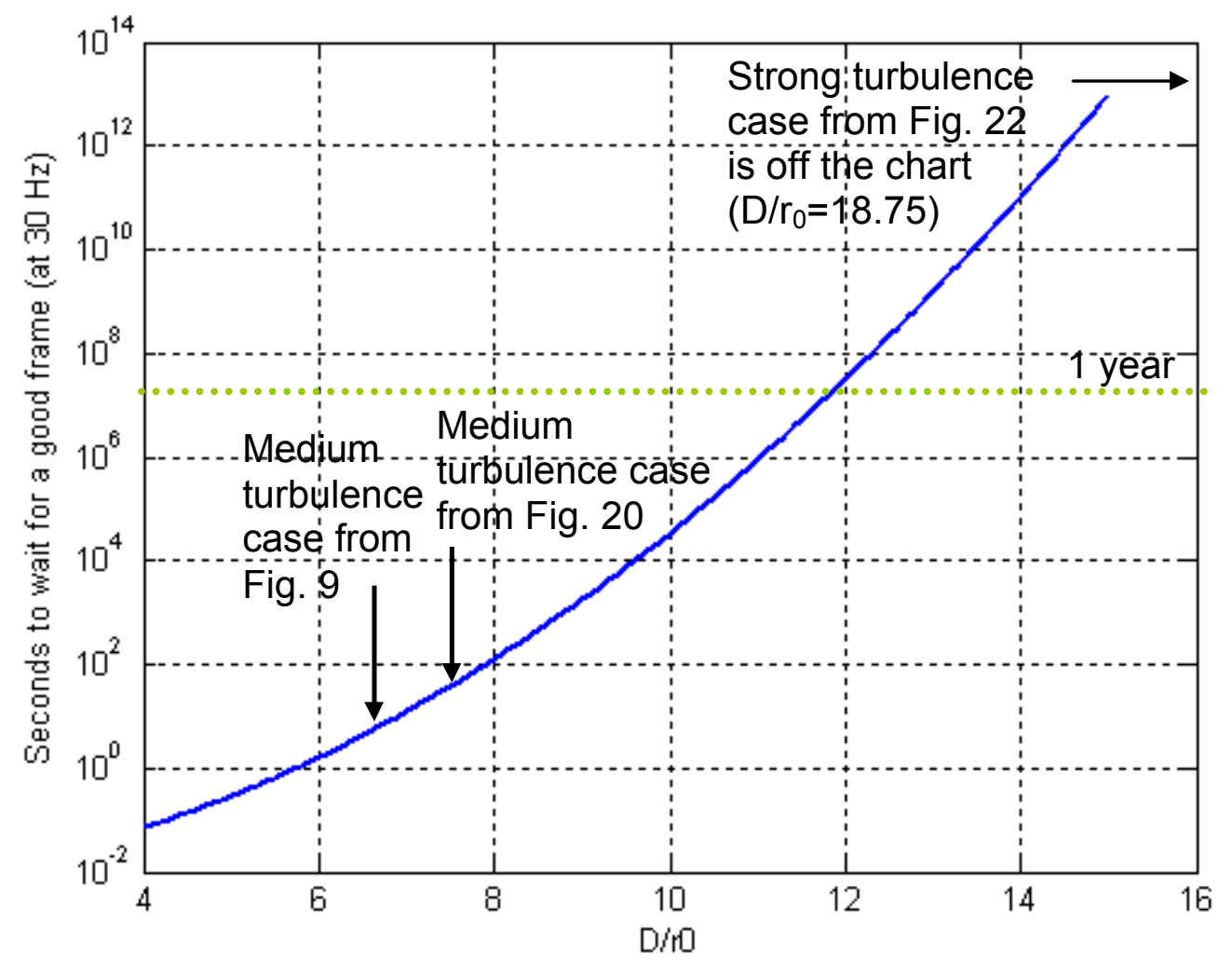

Figure 31: Wait time at $30 \mathrm{~Hz}$ between "good" frames or "good" regions as a function of aperture diameter to atmospheric coherence length. $\left(D / r_{0}\right)$

\section{References:}

[1] C. Carrano, "Speckle Imaging over horizontal paths", Proc SPIE 4825, pp 109-120, (2002)

[2] C. Carrano, "Anisoplanatic performance of horizontal-path speckle imaging", Proc. Of SPIE, Vol 5162, pp 14-27, 2003

[3] D. Fried, "Probability of getting a lucky short-exposure image through turbulence", JOSA, Vol. 68, No. 12, 1978 